Secondary Wireless Mesh Network Design Using

Leased Frequency Spectra 


\section{SECONDARY WIRELESS MESH NETWORK DESIGN USING LEASED FREQUENCY SPECTRA}

BY

SIAMAK SHAKERI, B.Sc.

A THESIS

SUBMITTED TO THE DEPARTMENT OF ELECTRICAL \& COMPUTER ENGINEERING AND THE SCHOOL OF GRADUATE STUDIES OF MCMASTER UNIVERSITY

IN PARTIAL FULFILMENT OF THE REQUIREMENTS

FOR THE DEGREE OF

Master of Applied Science

(c) Copyright by Siamak Shakeri, September 2010

All Rights Reserved 
Master of Applied Science (2010)

(Electrical \& Computer Engineering)
McMaster University

Hamilton, Ontario, Canada

TITLE:

Secondary Wireless Mesh Network Design Using Leased

Frequency Spectra

AUTHOR:

Siamak Shakeri

B.Sc., (Electrical Engineering) McMaster University,

Hamilton, Canada

SUPERVISOR:

Dr. Terence D.Todd

NUMBER OF PAGES: xii, 102 
To my mother and father, Sarah and Samad. 


\section{Abstract}

This thesis considers the design of secondary wireless mesh networks which use leased frequency channels. Considering the growing interest in using wireless services and the scarcity of frequency bands with proper propagation characteristics, reusing the currently licensed and underutilized bands is promising. A new initiative by the FCC, which allows the secondary usage of TV bands has encouraged both academia and industry to develop new devices and standards suitable for these operations.

This thesis considers the design of secondary wireless mesh networks which use leased frequency channels. In a given geographic region the available channels are individually priced and leased exclusively. The usage of each channel is also subject to published interference constraints so that the primary user is not adversely affected. When the network is designed and deployed, the secondary user would like to minimize the costs of using the required resources while satisfying its own traffic and interference requirements. This problem is formulated as a mixed integer optimization which gives the optimum deployment cost as a function of the secondary node positioning, routing, and frequency allocations. Because of the problem's complexity, the optimum result can only be found for small problem sizes. To accommodate more practical deployments, four algorithms are proposed and their performance is compared to solutions obtained from the optimization. 


\section{Acknowledgements}

I am heartily thankful to my supervisor, Terence D.Todd, whose encouragement, guidance and support from the initial to the final level enabled me to understand how to perform a research project. He was always there to listen to me and give me advice, and made my first research experience interesting.

I would like to thank Amir A.Sayegh, because of his support and collaboration during the project. I learned from him how to brainstorm a new idea, explore its validity and originality, and put it into a scientific format. This project wouldn't have been possible without his help and dedication.

I am grateful to all my friends in the Wireless Networking Laboratory of McMaster University, who not only helped me by their sharing of ideas, but also by maintaining a friendly environment that made the hard work more fun, Amir Sayegh, Ali Shariat, Keivan Rezaee, Mohammed Sheikh Zefreh, Ghada Badawy, Abdallah Hammad, Zhongliang Liang, Shan Feng, Wenjuan Liu and all my other friends in the ECE department of McMaster Unviersity.

Finally, I'm grateful to the administrative staff of the ECE department, especially Cheryl Gies, who were always helpful when advising me of the official requirements of my education at McMaster University. 


\section{Notation and abbreviations}

$B S$

$C B P$

$C P E$

$C T$

$D S$

$D S A$

EIRP

FCC

$G A$

IEEE

kbps

$L A N$
Base Station

Coexistence Beacon Protocol

Costumer Premise Equipment

Cognitive Transmitter

Down Stream

Dynamic Spectrum Access

Effective Isotropic Radiated Power

Federal Communications Commission

Genetic Algorithm

Institute of Electrical and Electronics Engineers

Kilobits Per Second

Local Area Network 
$M A C$

$M A N$

NP

OFDM

$P A N$

$P D U$

PHY

$R A N$

$R L$

$S C H$

SNR

TV

$T V B D$

$U S$

$W A N$

WLAN

WRAN
Medium Access Control

Metropolitan Area Network

Nondeterministic Polynomial

Orthogonal Frequency Division Multiplexing

Personal Area Network

Protocol Data Unit

Physical

Rural Area Network

Reinforced Learning

Superframe Control Header

Signal to Noise Ratio

Television

TV Band Device

Up Stream

Wide Area Network

Wireless Local Area Network

Wireless Rural Area Network 


\section{Contents}

Abstract $\quad$ iv

Acknowledgements $\quad$ V

1 Introduction 1

1.1 Dynamic Spectrum Sharing and Cognitive Networks . . . . . . . . . . 1

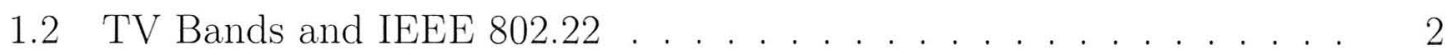

1.3 Pricing and Interference Constraint . . . . . . . . . . . . . . . . . 3

1.4 Optimization Problem And Computational Complexity . . . . . . . . 5

1.5 Organization of The Thesis . . . . . . . . . . . . . . . . 6

2 Dynamic Spectrum Access And Spectrum Sharing $\quad 7$

2.1 Introduction . . . . . . . . . . . . . . . . . . . 7

2.2 Current Standards And Government Mandates . . . . . . . . . . . . 8

2.2.1 FCC's New Report And Order Towards Reusing White Spaces in TV bands . . . . . . . . . . . . . . . . . 8

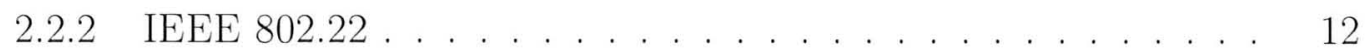

2.3 Centralized Spectrum Sharing . . . . . . . . . . . . . . . . 17

2.4 Distributed Spectrum Sharing . . . . . . . . . . . . . . . . . . . . 22 
2.5 Cooperative Spectrum Sharing . . . . . . . . . . . . . . . . . . . . . 28

2.6 Non-cooperative Spectrum Sharing . . . . . . . . . . . . . . . 32

2.7 Pricing in Dynamic Spectrum Sharing . . . . . . . . . . . . . . . 36

3 Secondary Wireless Mesh Network Design Using Leased Frequency $\begin{array}{ll}\text { Channels } & 40\end{array}$

3.1 Introduction . . . . . . . . . . . . . . . . . . . . 40

3.2 Related Work . . . . . . . . . . . . . . . . . . . 42

3.3 Problem Formulation . . . . . . . . . . . . . . . . . 44

3.4 Proposed Algorithms . . . . . . . . . . . . . . . . . . . . 52

4 Results $\quad 60$

4.1 Primary Network Topologies and Parameters: Static Scenario . . . 60

4.2 Tables and Comparisons . . . . . . . . . . . . . . . . 62

4.3 Primary Network Topologies and Parameters: Dynamic Scenario . . . 66

4.4 Tables and Comparisons . . . . . . . . . . . . . . . . . . . . 68

4.4.1 Overall Cost Tables ... . . . . . . . . . . . . . . . 68

4.4 .2 Number of Hops Tables. . . . . . . . . . . . . . . 76

4.4 .3 Number of Deployed Nodes Tables . . . . . . . . . . . . 83

5 Conclusions and Future Work 90 


\section{List of Tables}

4.1 MHFS Overall Cost . . . . . . . . . . . . . . . . . . 62

4.2 Greedy Flow-Based Overall Cost . . . . . . . . . . . . . . . . . . 63

4.3 Optimal Overall Cost . . . . . . . . . . . . . . . . 63

4.4 GFB and MHFS Comparison for Varying Network Sizes . . . . . . . 63

4.5 GFB: Overall Cost In Fully Connected Topology . . . . . . . . . . . . 68

4.6 GFB: Overall Cost In Random Topology . . . . . . . . . . . . . . . . 68

4.7 MHFS: Overall Cost In Fully Connected Topology . . . . . . . . . . . 69

4.8 MHFS: Overall Cost In Random Topology . . . . . . . . . . . . . . . 69

4.9 MHFSTS: Overall Cost In Fully Connected Topology . . . . . . . . . 70

4.10 MHFSTS: Overall Cost In Random Topology . . . . . . . . . . . . . 70

4.11 Dijkstra-ILS: Overall Cost In Fully Connected Topology . . . . . . . 71

4.12 Dijkstra-ILS: Overall Cost In Random Topology . . . . . . . . . . . . 71

4.13 GFB: Number Of Hops In Fully Connected Topology . . . . . . . . . 76

4.14 GFB: Number Of Hops In Random Topology . . . . . . . . . . . . . 76

4.15 MHFS: Number Of Hops In Fully Connected Topology . . . . . . . . 77

4.16 MHFS: Number Of Hops In Random Topology . . . . . . . . . . . . . 77

4.17 MHFSTS: Number Of Hops In Fully Connected Topology . . . . . . . 78

4.18 MHFSTS: Number Of Hops In Random Topology . . . . . . . . . . . 78 
4.19 Dijkstra-ILS: Number Of Hops In Fully Connected Topology . . . . . 79

4.20 Dijkstra-ILS: Number Of Hops In Random Topology . . . . . . . . . 79

4.21 GFB: Number Of Deployed Nodes In Fully Connected Topology . . . 83

4.22 GFB: Number Of Deployed Nodes In Random Topology . . . . . . . 83

4.23 MHFS: Number Of Deployed Nodes In Fully Connected Topology . . 84

4.24 MHFS: Number Of Deployed Nodes In Random Topology . . . . . . . 84

4.25 MHFSTS: Number Of Deployed Nodes In Fully Connected Topology 85

4.26 MHFSTS: Number Of Deployed Nodes In Random Topology . . . . . 85

4.27 Dijkstra-ILS: Number Of Deployed Nodes In Fully Connected Topology 86

4.28 Dijkstra-ILS: Number Of Deployed Nodes In Random Topology . . . 86 


\section{List of Figures}

2.1 WRANs Coverage Range Compared To Other Wireless Technologies[?] 14

2.2 Superframe Structure Proposed For IEEE 802.22 [?] . . . . . . . . . . . 15

2.3 Hidden Incumbent Problem . . . . . . . . . . . . . . . . . . . 16

2.4 Centralized Spectrum Sharing Concept . . . . . . . . . . . . . . . . . 21

2.5 Distributed Spectrum Sharing Concept . . . . . . . . . . . . . . . . . 27

3.1 Primary And Secondary Networks . . . . . . . . . . . . . . . . . . . . 44

4.1 Secondary Network Deployment Over Primary Network . . . . . . . . 62

4.2 Secondary Network Deployment Over Primary Network . . . . . . . . 66

4.3 Secondary Candidate Nodes Over Primary Network In Random Mesh Topology . . . . . . . . . . . . . . . . . . . 67

4.4 Overall Cost Of Deploying Secondary Network . . . . . . . . . . . . . 72

4.5 Overall Cost Of Deploying Secondary Network . . . . . . . . . . . . . 73

4.6 Total Number Of Hops For Routing Secondary Network Traffic . . . . 80

4.7 Total Number Of Hops For Routing Secondary Network Traffic . . . . 81

4.8 Total Number Of Deployed Secondary Nodes . . . . . . . . . . . . . . 87

4.9 Total Number Of Deployed Secondary Nodes . . . . . . . . . . . . . . 88 


\section{Chapter 1}

\section{Introduction}

\subsection{Dynamic Spectrum Sharing and Cognitive Net- works}

With the emergence of new wireless technologies, the need for vacant spectrum bands has become a major issue, considering the fact that most of the spectrum bands below $5 \mathrm{GHz}$ are already being licensed for the exclusive use of technologies such as GSM, TV, etc. Surveys on spectrum utilization [1] show that most of the licensed spectrum bands are underutilized. For this reason, increasing the utilization of these bands seems promising for solving the spectrum scarcity problem.

One way to approach this problem is using opportunistic and dynamic spectrum access methods with frequency agile radio devices. These types of networks are known as Cognitive Radio Networks. The FCC's newly adopted rules regarding the unlicensed use of TV white spaces[2][1] made the use of cognitive radios, as secondary users in TV licensed bands, completely legal. Now the companies working on wireless networks 
have the opportunity to make use of these rules, considering the static nature of the TV bands, which reduces the complexity of these secondary networks.

Various spectrum sharing schemes are proposed throughout the literature, which can be used according to the network topology, performance constraints, as well as other design parameters. This can be centralized, where a central server coordinates between the secondary users, or distributed, in which users decide locally to use or vacate a channel. In cooperative scenarios, secondary users try to come to an agreement before starting to operate, which helps reduce collisions among the users, thus leading to better QoS, while in non-cooperative scenarios, spectrum allocation is performed by every node independently, without considering its effect on the overall network performance.

\subsection{TV Bands and IEEE 802.22}

The US Federal Communications Commission (FCC), released rules on November 14, 2008 regarding unlicensed operation in TV Bands [2][1]. These new rules are the first official reaction to cognitive radio technology. In this report, a number of constraints on the performance of the TV Band Devices (TVBDs) are mentioned, to guarantee the uninterrupted operation of licensed on TV band devices. Minimum required SNR in spectrum sensing, existence of a central database in the secondary network, as well as recognizing different operating modes for secondary networks, are among the requirements in the newly issued report.

In the FCC report, geographical information about all licensed TV Band services are stored in a database, which makes it easier to find an unused channel in a specific region. All the secondary TVBDs should be registered in the database, so that any 
violation can be easily identified.

In response to this report and the growing interest in utilizing these bands on an unlicensed basis[3], IEEE 802.22, the Working Group on Wireless Regional Area Networks ("WRANs"), was established. IEEE 802.22's primary objective is to develop standards for a cognitive radio-based PHY/MAC/air interface to be used by unlicensed devices on a non-interfering basis in the spectrum that is allocated to the TV broadcast services. So far, Draft v3.0 of this new standard is issued which encompasses task groups on PHY, MAC and security, co-existence and cognitive radio capabilities. These official standards can be used as a basis for both academic research, as well as industry innovation around the secondary use of licensed bands. In this thesis, a spectrum sharing scheme consistent with the FCC's order is proposed, where the cumulative interference is accurately controlled to make sure that regulations are not violated.

\subsection{Pricing and Interference Constraint}

The idea of pricing in secondary cognitive networks has been proposed in various scenarios. One way is to use pricing as a punishment for violations, e.g., interference. In this case[4][5], the secondary network is being charged when the interference constraints are being violated. This ensures that secondary network tries to minimize its interference over the primary links.

Another mechanism is to charge the secondary user for using the primary's licensed frequencies. In this scenario, the primary network would have an incentive to allow and cooperate with the secondary network. Market-equilibrium, competitive, and cooperative pricing models have been proposed in the literature [6],[7]. Game Theory 
based pricing is also useful when secondary and primary users try to reach a pricing scheme as the players of the game, before starting to share the spectrum[8]. The pricing scheme developed in this thesis is in the second category, where the secondary users is charged for their spectrum usage, thus the secondary network is driven to better utilize its spectrum usage in order to reduce its costs.

One of the major concerns in the secondary use of licensed bands is the interference that such operation will introduce over the primary links. "Interference Temperature" is introduced as the cumulative interference over each primary link[9][10], or in other words, interference is controlled and calculated on the receiver's side. Controlling this parameter would ensure the desirable operation of the primary network. Various power control schemes to maximize the energy efficiency of the secondary users and guarantee the QoS of both the primary users and the secondary users have been explored. The feasibility condition of these problems are derived for both centralized and distributed scenarios[11][12][13].

A different approach to tackle this problem is to penalize the secondary network for the violations of the interference constraints[5]. Although this approach seems promising, it can not guarantee the unaffected QoS of the primary user. In this thesis, we have used the interference temperature concept, where the cumulative interference over both primary and secondary links are constrained to be below a certain level, to ensure that the desired QoS of the both networks are guaranteed. 


\subsection{Optimization Problem And Computational Com- plexity}

The approach pursued in this thesis to design secondary networks is to formulate a joint routing node placement and frequency allocation optimization problem to minimize the cost of deploying the network, subject to the interference, link capacity and flow conservation constraints. It is also assumed that a primary network is already deployed, and its effect over the secondary network design is embedded in the interference constraints and the frequency prices. The problem is solved for both static and dynamic scenarios, where in dynamic scenarios, it is assumed that the secondary traffic matrix is changing over time and the optimization problem is performed to find the optimal routing, frequency allocation and node placements in every time slot. The formulated problem falls into the class of mixed integer optimization problems[14][15][16]. Due to the existence of integer variables, this problem is not convex [17]. One popular approach to find the optimum solution is to use the Branch and Bound Method[18]. The computation complexity of this method is exponential [18], thus the required time and resource may explode for large size networks. Therefore it is imperative to design heuristic algorithms which find suboptimal solutions with less computational complexity[19].

In this thesis, four heuristics are proposed. Three of them are still $O(\exp )$, but greatly reduce the size of problem by separating frequency allocation and routing phases, and doing the problem for the flows consecutively. A modified Iterated Local Search(ILS) approach is proposed to locally optimize the existing suboptimal solutions[20]. Using Dijkstra's Shortest Path Algorithm, and performing a modified 
ILS, together shape the most efficient heuristic that is proposed with the computational complexity of quadratic order, by which a larger scope of network sizes and scenarios can be investigated.

\subsection{Organization of The Thesis}

The rest of this thesis is organized as follows. Chapter 2 begins by discussing both the FCC's newly adopted regulations, as well as the proposals by the IEEE 802.22 working group. In the remaining sections of this chapter, various spectrum sharing scenarios are investigated and the current articles on these issues are discussed. The pros and cons of each method are explored and their performance is compared. In Chapter 3, the design of a secondary wireless mesh network with the capability of operating over TV band frequencies is proposed. The resulting mixed integer optimization problem is formulated and its computational complexity is discussed. Four heuristic methods are proposed to tackle the problem of the increasing computational size of the design. Three of the heuristics still have an exponential worst-case computation size, but since they divide the problem into smaller size ones, they perform much faster. A heuristic algorithm based on Dijkstra's Shortest Path Algorithm is proposed with quadratic order computation size increase, in the worst case. Chapter 4 is dedicated to comparing the results gained from solving the optimization problem for both the original problem and the heuristics. Two different primary network topologies are tested to show the merits of the heuristics under highly and moderately crowded primary networks. Overall cost, number of hops, as well as the number of deployed nodes of the designed secondary networks are compared. Finally, conclusions of the thesis are made in Chapter 5. 


\section{Chapter 2}

\section{Dynamic Spectrum Access And Spectrum Sharing}

\subsection{Introduction}

Growing interest in the use of wireless technology has made the spectrum bands with desirable propagation characteristics scarce. The FCC's spectrum policy task force survey on spectrum usage indicates that in the spectrum below $1 \mathrm{GHz}$ in Atlanta, Chicago, New Orleans, San Diego, and in a Washington, and a DC suburb during various periods in July 2002, the average channel usage was less than 15\%, with the peak usage of approximately $85 \%$ [21].

After the US digitalization of the TV bands in 2009, the $700 \mathrm{MHZ}$ band was turned over to the government. In Auction 73, this band was to be sold with a minimum bidding price of more than 19 billion dollars[22]. These ten figure numbers and the underutilization of the currently used spectrum, with the FCC's new directive to allow the secondary usage of TV bands, which because of its static nature is much 
less complex than other bands, have stimulated both academia and industry to invest in reusing these bands on a secondary basis. Current static frequency allocation schemes are not able to work under the new order and thus new techniques should be envisioned to overcome this issue. Various ideas involving dynamic spectrum access and sharing have been proposed, which will be discussed in this chapter.

\subsection{Current Standards And Government Man- dates}

\subsubsection{FCC's New Report And Order Towards Reusing White Spaces in TV bands}

The Federal Communications Commission (FCC) released adopted rules regarding the unlicensed operation in TV bands on November 14, 2008 [1]. Although these rules are subject to change in future amendments, they provide a proper framework for designing secondary networks in the TV bands. In this section a brief discussion of this report is presented.

TV band licensed services: There are different TV band services known to be licensed in the frequency bands below 800 Mhz. Examples are full service TV stations, Class A TV stations, private land mobile radio service, medical telemetry equipment, etc. Wireless microphones also use the TV bands on an unlicensed basis, and their operation should be protected from interference caused by TVBDs (TV Band Devices).

Central Database: The FCC mandates the existence of a central database that 
contains the geolocational information about all licensed TV band services which operate over the TV bands using fixed transmitters with designated service areas. Places where wireless microphones are regularly used like sport venues should also be included in the database. Using the database information, a TVBD can easily find out which TV band channels are not used by licensed services in its operating area. This database is operated by a third party, and multiple administrators can set up a database and charge the TVBDs that have access to it. All the fixed TVBDs should be registered in the database.

Different Operating Modes: The FCC recognizes three different operating modes for devices operating in the TV band on a secondary basis:

- Fixed Devices: These devices have fixed and determined locations. Their location and the owner's contact information should be registered in the central database. This will help finding the source of interference on the TV band licensees. These devices should access the database at least once a day to update their information about available TV band channels. They should be equipped with a geolocation positioning system like GPS. They are allowed to transmit at maximum power of $1 \mathrm{~W}$ and $4 \mathrm{~W}$ EIRP.

- Portable/Personal Mode 2: These devices can be portable devices like PDAs or laptops. They operate independently, but they should have a geolocation positioning system and access to the database over the Internet. They can transmit at maximum power of $50 \mathrm{~mW}$ EIRP and are not required to be registered in the database.

- Portable/Personal Mode 1: These devices can be again PDAs or laptops. They don't need to have access to the database or a geolocation positioning system. They also don't need to register in the database, but their operation should be completely 
controlled by a fixed or portable/personal mode 2 device. In other words, they work on a client basis. Their transmission power should not exceed $100 \mathrm{~mW}$ EIRP.

Spectrum Sensing: All TVBDs should be equipped with spectrum sensing features in order to guarantee non-interference operation in TV bands. Existence of a spectrum sensing feature helps protect wireless microphone operation, since they are not registered in the database, work at low signal levels, and occasionally change their location.

Spectrum Sensing Level: All TVBDs are required to have the ability to detect licensed operations at least at $-114 \mathrm{dBm} W$ level.

Transition Time Requirements: Every TVBD should sense the channel that it decides to transmit on, for at least 30 seconds. TVBDs should also recheck the channel every at least 1 minute to make sure it is still available. Whenever a TVBD senses the operation of a licensed device, it should vacate the channel within two seconds.

Laboratory Check: Every TVBD should be tested in an FCC designated laboratory to make sure the device satisfies the required constraints.

Wireless Microphone Protection: Since wireless microphones are one of the TV band licensees, the FCC has enforced certain constraints to make sure they are protected from unlicensed operation. The FCC requires "The locations where wireless microphones are used, such as entertainment venues and for sporting events, can be registered in the database and will be protected as for other services. In addition, channels from 2-20 will be restricted to fixed devices, and we anticipate that many of these channels will remain available for wireless microphones that operate on an itinerant basis. In addition, in 13 major markets where certain channels between 14 
and 20 are used for land mobile operations, we will leave two channels between 21 and 51 free of new unlicensed devices and therefore available for wireless microphones. Finally, as noted above, we have required that devices also include the ability to listen to the airwaves to sense wireless microphones as an additional measure of protection for these devices".

SNR required for Spectrum Sensing: As mentioned earlier, FCC requires TVBDs to have the ability of detecting licensed users at $-114 \mathrm{dBm}$ W levels. Here, we calculate the required SNR in sensing devices.

Thermal noise power in a $50 \mathrm{ohm}$ system can be calculated using the following formula:

$$
\text { PNoise }=k T B
$$

Where $B$ is the bandwidth $=6 \mathrm{Mhz}$ (every TV channel), $\mathrm{T}=300 \mathrm{k}$ is the temperature, and $\mathrm{k}$ as the Boltzmanns constant. So thermal noise power would be:

$$
\text { PNoise }=-106.22 d B m W
$$

comparing the above noise in a TV channel and -114 $\mathrm{dBmW}$ that FCC requires, spectrum detectors should be able to detect at $-8 \mathrm{~dB}$ SNR levels. We should also consider the fact that there are other noise sources that can increase the noise floor, so we need to reach sensing levels near $(-10)$ to $(-15)$ dB SNR levels. As can be seen from the above statements, the FCC has taken a conservative approach to highly protect the TV band licensed operations. This is mainly because the new technology has not been put into practice to see how reliable it can be. So the Federal Communications Commission has tried to guarantee the non-interferening operation of TVBDs. 
However, as it is mentioned in the order and report, there may be future relaxed constraints, probably only requiring spectrum sensing in currently low SNR levels.

The model that is presented in this thesis follows the above constraints. It is assumed that the available frequencies for secondary network usage are predetermined, which easily follows from the existence of a central database. The placed nodes have fixed locations and their operating frequencies are predetermined by inquiring from the central database. And above all, interference is vigorously controlled at any desired level, so that there would be the least harmful effect on the TV services. In the next chapter we further discuss the model.

\subsubsection{IEEE 802.22}

Considering the need for broadband Internet access in rural areas, and the desirable propagation characteristics of the TV bands, which are permitted to be used on a secondary basis, IEEE established the 802.22 working group[23] for Wireless Rural Area Networks (WRANs) with the responsibility for design standards for PHY/MAC/air interfaces as well as co-existence and other issues that are related to the operation of so-called TV Band Devices (TVBDs)[24][25]. Currently the existing IEEE 802.22 standard is in a draft version and subject to change according to proposals from various corporations. In this section a brief description of the current proposals is discussed.

IEEE 802.22 proposes a point to multipoint communication consisting of Base Stations and Customer Premise Equipment (CPE's), where every BS controls the communications in its surrounding cell. Every CPE in a cell is controlled by its BS for accessing the medium. Due to the existence of a primary network (TV band Services) 
in the operating channels, BSs are also responsible for maintaining a distributed spectrum sensing scheme to avoid interference with licensees. In fact this role reflects the FCC order and report towards portable devices mode 1.

The operating band is confined to 54-862 MHZ, and channel bandwidths of 6, 7 and $8 \mathrm{MHz}$ are proposed. The downstream bit rate is expected to be 18-24 Mbps. OFDM is used in the PHY level and considering the sharing of a channel between 12 users [25], DS bit rate for each CPE would be from 1.5 Mbps. US is expected to reach close to $300 \mathrm{kbps}[?]$.

Due to desirable propagation characteristics of the band and the relatively high power transmission of CPEs, which is proposed to be 4 Watts EIRP, WRAN cell range can span to $33 \mathrm{~km}$. With proper scheduling and compensating for the propagation delay by the MAC layer, this range can be increased to $100 \mathrm{~km}$, as shown in Figure 2.1 .

The IEEE 802.22 MAC uses a superframe structure for efficient data transmission as well as cognitive functionalities such as incumbent detection and spectrum sensing. As depicted in Figure 2.2, a superframe starts with a preamble which includes a super frame control header(SCH) followed by a number of MAC frames (16 frames in [24]).

A similar approach to the FCC's order and report is pursued here in setting up a database, where the available TV channel is recorded and both CPE's and BS's are registered so that any undesirable interference from any WRANs can be identified.[24].

\section{Hidden Incumbent Systems}

In addition to referring to the database, which records the existing licensees in every region, spectrum sensing done by the secondary users is an important source of 
information for the BSs to determine candidate operating channels. A major problem that may occur is that the secondary users in the interference or overlap region fail to report the existence of an incumbent to their corresponding BS, e.g., if the secondary users (or CPEs) are under strong interference from the incumbent, they cannot receive the proper signaling from their BS to report the interference. Thus the BS will assume the occupied channel as a valid candidate. Figure 2.3 depicts this scenario. Secondary users in the interference region may be unable to report the primary operation in that region.

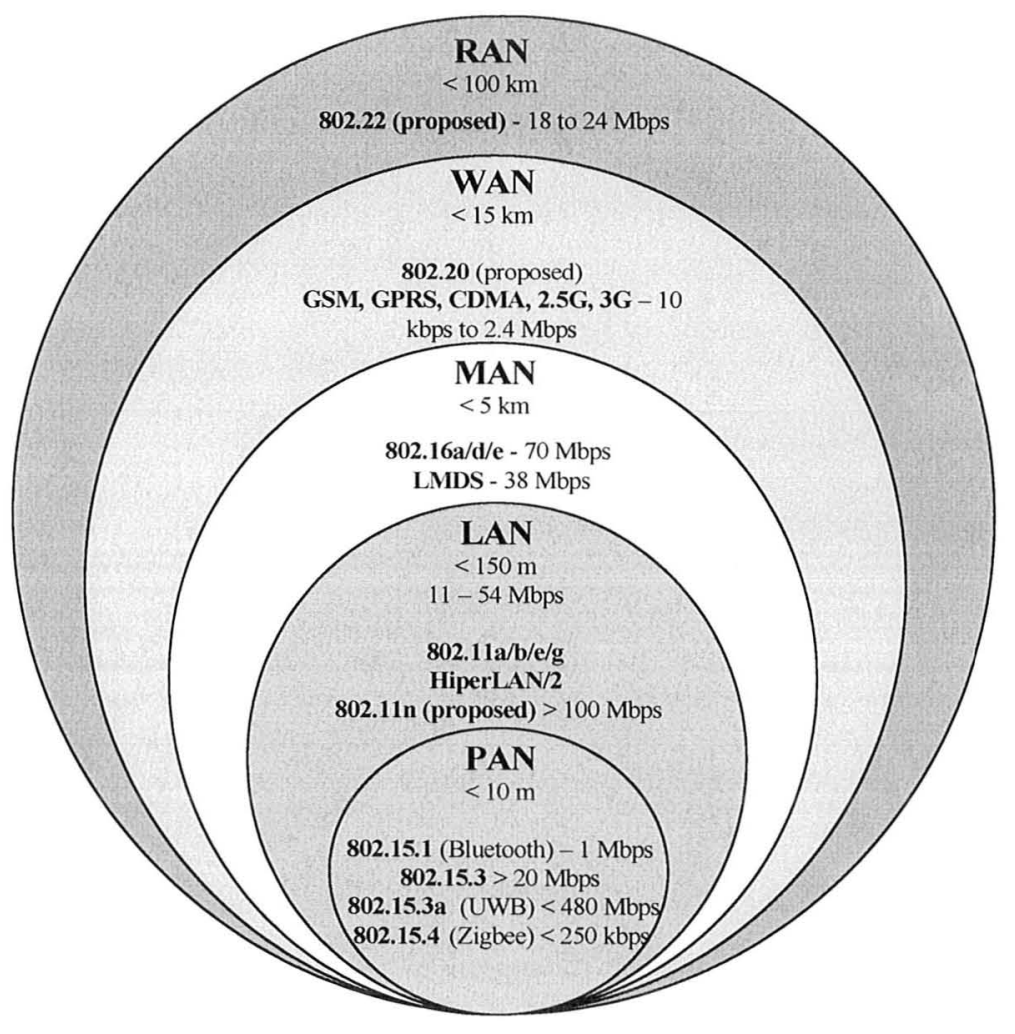

Figure 2.1: WRANs Coverage Range Compared To Other Wireless Technologies[?] 


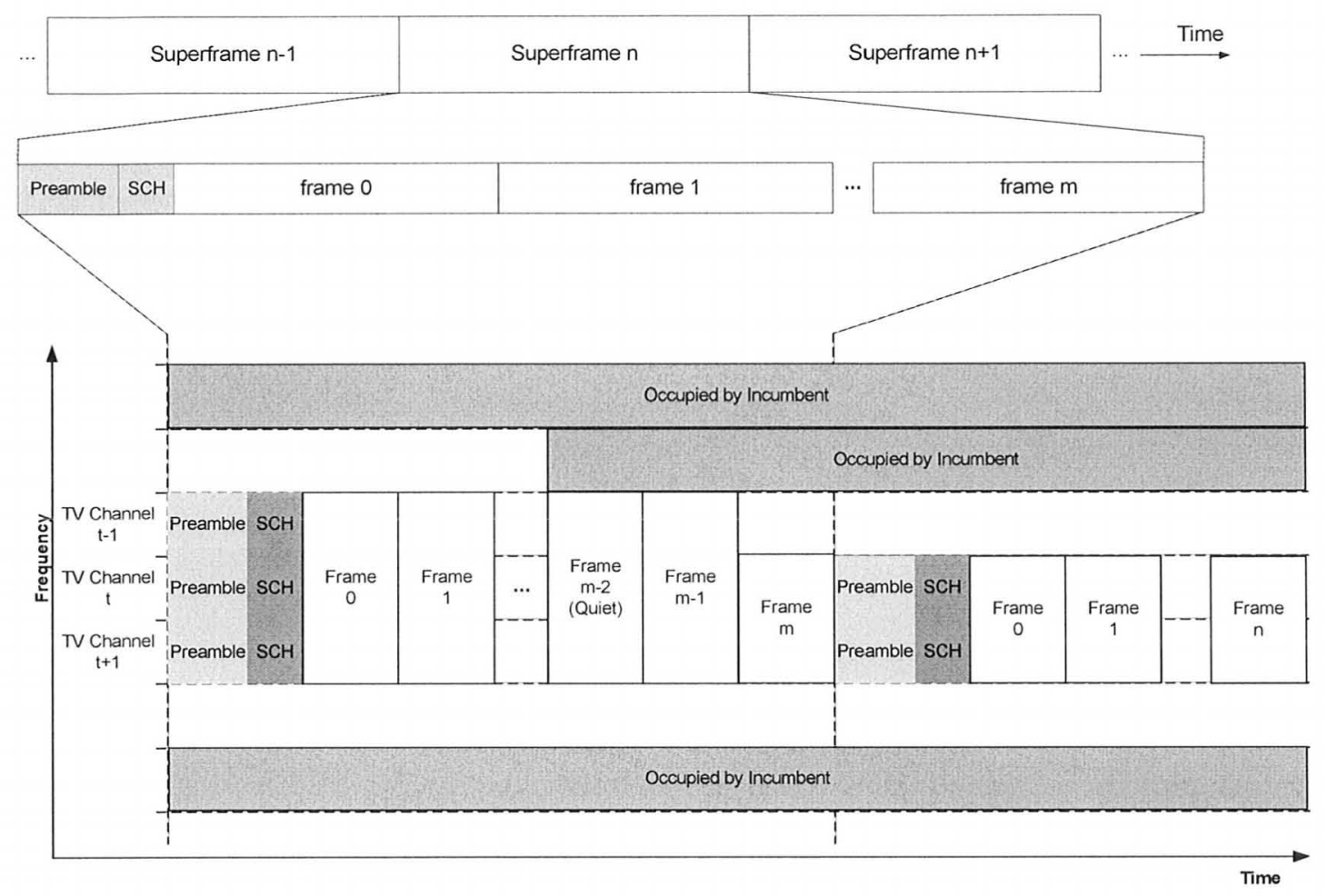

Figure 2.2: Superframe Structure Proposed For IEEE $802.22[?]$

Both implicit and explicit approaches are proposed to tackle this problem. In the implicit approach [?], the BS periodically sends sensing requests to its CPEs and if it doesn't hear their responses after a waiting time, the channel is assumed to be unaccessible. In this approach, if secondary CPEs in any region are turned off, their corresponding BS assumes their operating channel is occupied by an incumbent, which is not always the case. The explicit approach solves this issue by broadcasting the sensing request calls for one specific channel over other candidate channels. In this case if the CPEs are in the interference region and turned on, they can report the primary (incumbent) existence in that specific channel. This method is called out-of-band sensing and is envisioned in the super frame structure by setting a flag 
in the $\mathrm{SCH}$ to differentiate between regular service MAC frame and the out-of-band sensing broadcast frame.

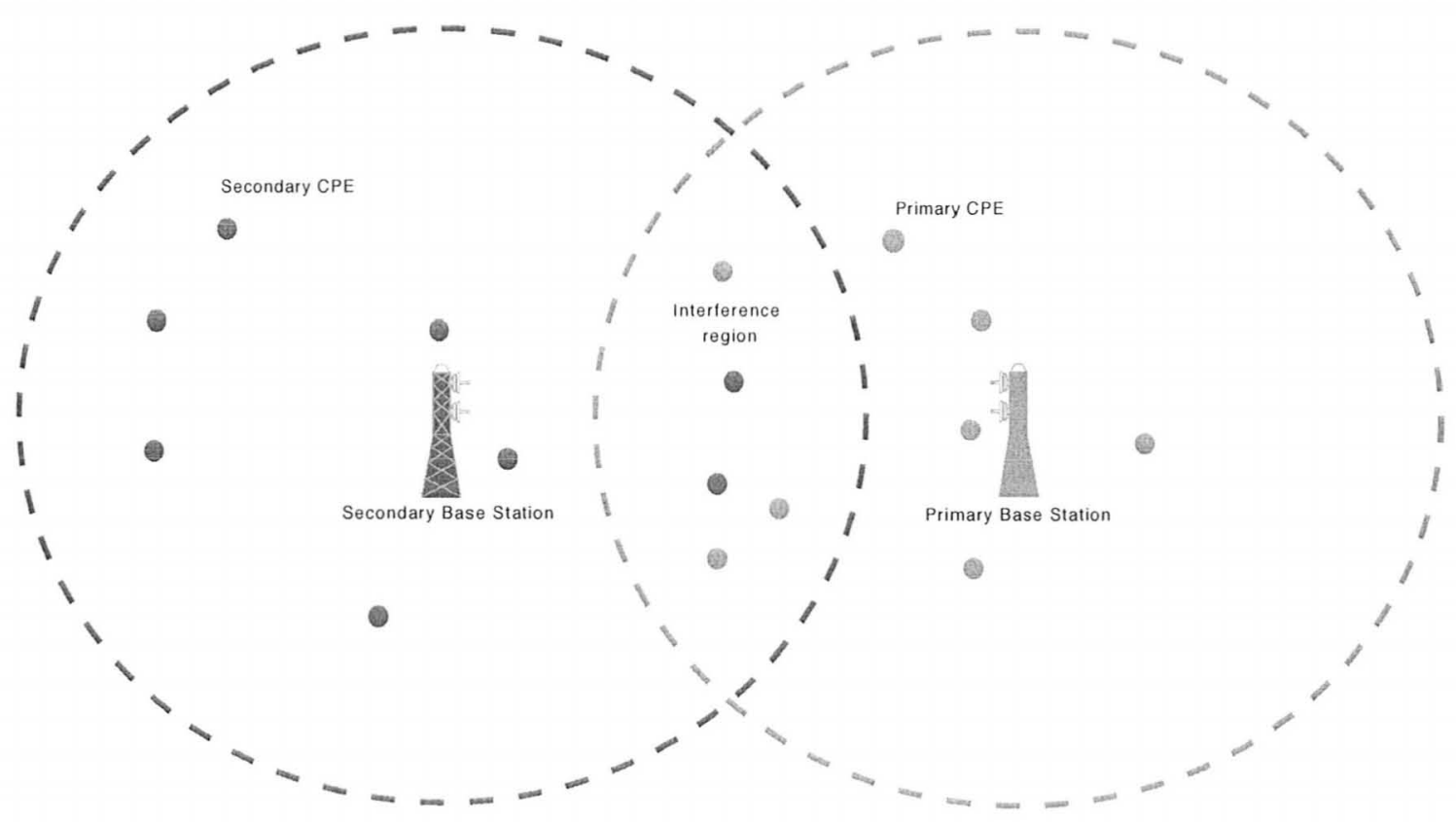

Figure 2.3: Hidden Incumbent Problem

\section{Coexistence}

Contrary to licensed services, where frequency channels are dedicated to a certain licensee, and the licensee can decide how to allocate its available channels among the network cells, in WRANs, neighbor cells use the frequency channel opportunistically. Thus the coexistence between the licensees and secondary users, as well as within secondary users (self-coexistence) should be controlled. Besides the government mandates regarding the spectrum sensing, which should be performed to avoid interference, MAC solutions are also proposed to solve these problems.

The authors in [?] propose an auctioning protocol, where the licensee plays the role of the offerer and the secondary BSs as the renter. Both offerer and renters 
are allocated a predetermined number of tokens called Credit Tokens (CT). Sharing is preformed dynamically when a renter and the offerer exchange tokens. This is performed dynamically and ensures both fairness and coordination between the secondary and primary base stations and doesn't represent money. Reference [26] models this credit token based protocol as a game, where base stations, as the players, try to optimize their own utility function until the Nash Equilibrium is achieved, where no contender can further increase its utility function.

Due to the opportunistic nature of spectrum sharing there is a chance that two neighbor WRAN cells use the same channel. Therefore there should be a method to control the self co-existence of WRANs. Base station beacon based and Co-existence Beacon Protocol(CBP) solutions are proposed in [24]. In the base station based solution, WRAN BSs use beacons to synchronize and coordinate the shared medium. CBP is another solution performed by CPEs which are then controlled by BSs. In this scenario, CPEs send out packets containing information about their surrounding cell and their corresponding BS. During a Self-Coexistence Window (SCW), which is synchronized through the operating channels, a superframe consisting of SCH and CBP PDUs is being transmitted over the shared medium for coordinating the coexitance and spectrum sharing.

\subsection{Centralized Spectrum Sharing}

One way to share frequency bands among secondary users is to assign a centralized server or spectrum manager responsible for allocating and coordinating the shared resources among the network users[27][28]. Spectrum sensing information gathered by CPEs are sent to the central entity, where decisions regarding channel access are being 
made. This approach complies with the FCC's requirement regarding the existence of a central database. In this case the manager can take the role of the database, and thus the overhead introduced by the exchange of information between the database and the central entity would be removed. In the following paragraphs an overview of the proposed methods based on this generic centralized scheme is presented.

The authors in [29] try to maximize the minimum QoS of cells and improve the spectral utilization by reusing the available frequency bands of two networks with shared resources. A central optimization problem is introduced, where the utility function based on the bandwidth request and satisfaction of each cell is being maximized. Restrictions are applied in the form of a compatibility matrix, which determines if two cells can reuse a specific frequency. It is claimed that finding the optimal solution becomes an NP-hard problem, thus heuristics are designed to reduce the computation size.

Reference [30] proposes another centralized spectrum reuse approach. In this paper a situation is investigated where two operators in a cell trade their licensed spectrum when one network has low traffic and the other is experiencing a heavy load. In trading periods, which are prearranged, short term frequency trading is performed until the next trading period, when a new arrangement may be achieved. In all the trading periods the initial allocation is the long-term government-mandated one. It is arbitrary for each of the networks to take part in the trade. Prior to entering each trading period, every network has an estimate of its required resources for the time slots following that period. This two-level hierarchical approach is further developed by deploying decentralized game theory-based algorithms, or in other words in a hybrid manner. 
Spectrum pooling is another spectrum sharing scheme investigated in [31]. In this approach, where OFDM is an appropriate candidate for the secondary network, the idea is to match the unused spectrum of the licensed operator with the subcarriers of the secondary network. Due to the exclusive nature of sharing, minimal interference would be experienced. Silence periods are scheduled, during which no secondary user transmits and unused frequency band detection is performed. An important issue is synchronization, which is critical in OFDM systems. Interference that occurs when primary users want to use their sub-bands, which are occupied by the secondary, can cause frequency offset in the OFDM system. A scheme based on using preamble/pilot with the adaptive filtering of the interference from the licensed users is proposed in this article.

Spectrum sharing among spread spectrum users is considered in [32]. SINR-based and power-based auction algorithms are proposed for spectrum allocation. In the auction process, first the manager determines a reserve bid and a price. After analyzing these values, every user submits its bid. After receiving the bids, the manager sets the allocated power to each user in a way that the received power in every user, which consists of a transmitter and receiver pair, is proportional to its bid. In SINR-auction, the user's payments are a function of both transmitted power and interference, while in the power-based option, it is only a function of the transmit power.

Reference [33] proposes and designs a complete bidding process in the MAC layers of the users. There is an Economical Manager responsible for performing the auction process. The good to be presented is the bandwidth for a specified time duration. At the start of the bidding process these goods are presented according to the available bandwidth. An auction agent responsible for bidding is located in the MAC layer 
of the users. These agents are responsible for estimating the needs of their corresponding users in the time period leading to the next bidding, as well as tracking the previous bidding prices to propose an improved bid. EM is responsible for predicting the available goods in the next time period, as well as the reserved price. The auction process is performed and controlled by the EM. In this scenario, the auction is performed in predetermined intervals, thus the agents in the MAC layers and EM should interact repeatedly.

Figure 2.4 depicts the concept of centralized spectrum sharing. In the upper figure, each group of CPEs are controlled by a base station and then the base stations are further controlled by a spectrum manager which performs the spectrum allocation and scheduling between various cells. Then in every cell, the corresponding BS performs the allocation task. In the lower figure, another scenario is depicted, where all the secondary CPEs are directly controlled by the spectrum manager. 

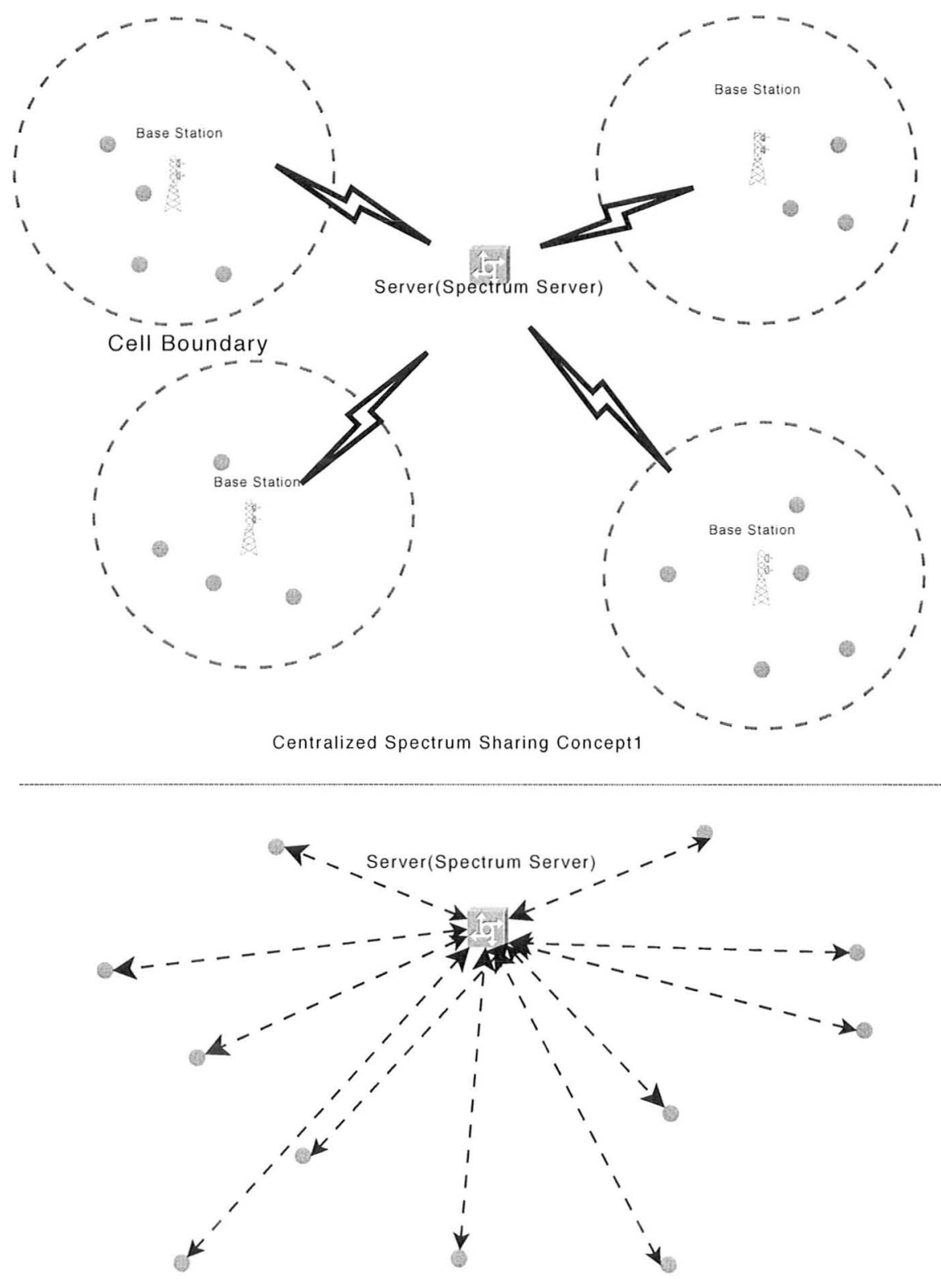

Centralized Spectrum Sharing Concept2

Figure 2.4: Centralized Spectrum Sharing Concept 
In the all various algorithms mentioned above, the key common property is the existence of a moderator or manager that performs the spectrum sharing. The users or customers of the network are only responsible for following the central entity's orders and in some cases, performing spectrum sensing and reporting the results to the manager. This method has several pros and cons. Using a central entity would ensure the unaffected operation of the primary network while being overlaid by a secondary operation, and it perfectly complies with the FCC's regulations referring to a database to check the availability of the spectrum. However this requires that the secondary network be structured and mandates setting up the infrastructures involved in the spectrum sharing. Thus it would be difficult to operate ad hoc and distributed networks, where users can independently find and reuse the unoccupied channels. On the other hand, the former introduces latency and overhead due to the constant monitoring of the channels by the manager and handshaking that should be performed prior to communication setup. In the next section, distributed spectrum sharing will be discussed, where the role of the central entity is reduced or completely removed.

\subsection{Distributed Spectrum Sharing}

A different scheme compared with the centralized spectrum sharing approach, is to perform the spectrum sharing and access process locally. In this scheme the secondary users or CPEs are not only responsible for spectrum sensing, but also have a decisive role in spectrum sharing decisions. This localized approach is very suitable for dynamic and distributed networks, since the decisions are taken locally, and even in some scenarios the individual secondary users sense, decide and reuse the spectrum 
independently.

Since each group of the users (or each of the users) work independently, the information sharing between the users, which helps them taking decisions that can be more optimal, becomes a challenge. The authors in [34] consider two different scenarios: "Public and Private Spectrum Information". In the public scenario the sensing information is broadcast over the network via base stations. Thus the behavior of the secondary users (or so-called cognitive users) would be symmetric. Models based on Game Theory are proposed to study this behavior, as well as a Nash Equilibrium which defines the mixed strategy equilibrium of the individual decisions of the secondary users. In this scenario, it is assumed that every user is aware of the decision of the other users. Since the sensing information is available for each one, the behavior of each user would be predictable. In the second scenario, only private sensing information is available for the users, and thus each user's decision is based on the sensing information performed by its own equipment. Authors have proposed the "Maximum Criterion" approach, where each user takes decisions assuming the others take the worst ones.

Game theory is a central concept in the problems associated with distributed spectrum sharing. Since the modeling of the secondary users as game players and their corresponding equilibrium decisions as a Nash Equilibrium would provide an accurate way of presenting and analyzing distributed spectrum sharing issues. Niyato et. al. in [35] model the sharing between a primary user and a number of secondary ones as an oligopoly market competition. A spectrum sharing solution is gained from a non-cooperative game and the resulting Nash Equilibrium. In the proposed scenario, secondary users gradually adjust their spectrum sharing strategies based on 
the previous decisions that they took and the consequences of such decisions.

The same authors in [36] have applied game theory to perform spectrum sharing and admission control for users operating in a heterogeneous environment consisting of wireless LAN, MAN and cellular networks. Three different games are designed to perform these tasks. The players in these games are the networks existing in the service area, contrary to the previous paper where players were the secondary users. First a noncooperative game is designed for the bandwidth allocation of the competing networks in the service area. The second game is used to give priority to vertical and horizontal handoffs over the new connections. This will guarantee a minimum QoS that should be maintained for various connections. The results from the Nash Equilibrium of the second game determines the amount of bandwidth offered to the new arrivals. The utility function is the profit that each network gains from the offered bandwidth. The role of game theory here is to coordinate between the players (heterogeneous networks) and to find the equilibrium, in which players' utility functions can not be further improved.

Another game theoretic spectrum sharing and power control scenario is discussed in [37]. In this model, the available spectrum is divided into orthogonal channels, and each wireless device can start transmitting into any number of the channels with any arbitrary power. The objective of the game is to achieve an equilibrium which is optimally fair as well as efficient. This work has shown that increasing the number of available channels, increases the efficiency, and at the same time convexifies the utility space, which makes finding the optimal allocation achievable.

Akyildiz et. al. in [38] discuss the idea of dynamic spectrum access in wireless mesh networks. In their distributed scheme, the secondary network operates over 
unlicensed bands, and if possible shifts to primary channels. Each secondary mesh user performs sensing of primary channels, while operating on secondary bands. These data are used to predict white spaces in the primary spectrum, but before starting the secondary operation in the primary bands, the estimated introduced interference is calculated to prevent harmful effects over the primary network. The task of channel assignment is performed at the routers, where given the local sensing information and the analytical results of the interference estimation, an optimization problem is performed to find the set of clusters to be shifted to the primary band.

Although the coordination overhead is reduced in a distributed manner, some is still required when the equilibrium is reached. In order to further reduce it, the authors in [39] propose a new approach. In their design, nodes based on their own observations decide independently to use the spectrum, but they are required to comply with a set of rules, which try to increase fairness and network utilization. A function called Poverty Line, which represents the minimum number of available channels, is defined for every node. The aforementioned rules force prescribed decisions in channel reuse or contentions based on the Poverty Line of each user. Thus the overhead would decrease due to less communication among nodes for channel coordination.

Reference [40] discusses another scheme where allocation rules are designed to coordinate among selfish and independent nodes. In this model the goal is to maximize utilization, without considering fairness. Thus there would be no guarantee that a minimum number of channels be allocated to each user. An innovation in this paper is that the allocation is performed sequentially upon the arrival of the users, and according to each situation that might happen, a rule is defined. If the newcomer demands a frequency band, and after the allocation of that band, no user in that 
band experiences above-threshold interference, the allocation is "seamless". If this condition (interference) doesn't stand, then the newcomer buys out the users that are already in the band that cause interference. Thus the allocation in this case would be exclusive. Another situation happens if the perceived interference of the newcomer is below the threshold but some existing users can't tolerate the increased spectrum. In this case which is called "defensive", the existing users try to buy out the newcomer. As can be seen, this algorithm performs poorly in providing fairness amongst users, and there may be no guarantee of QoS for the secondary network.

A distributed spectrum management scheme based on reinforced learning is proposed in [41]. A cellular concept is assumed, where in every cell the users are controlled by the base station, but the cells perform independently, thus a semidistributed approach is followed. Cell operation is categorized into short term and mid-term scheduling. In the short term case, the users are allocated the available channels in every time frame. In the latter, the scheduler decides which frequencies should and which shouldn't be used in the next ten seconds to ten minutes. The socalled Cell Dynamic Spectrum Access (DSA) Controller is implemented in each cell, thus allowing every cell to perform independently and autonomously from the others. A reinforced learning algorithm implemented in the DSA Controller is responsible for final spectrum allocation within the cell. After each assignment, the RL-DSA algorithm receives a reward proportional to the result of its previous assignment, that allows the RS to learn the appropriate allocation. 


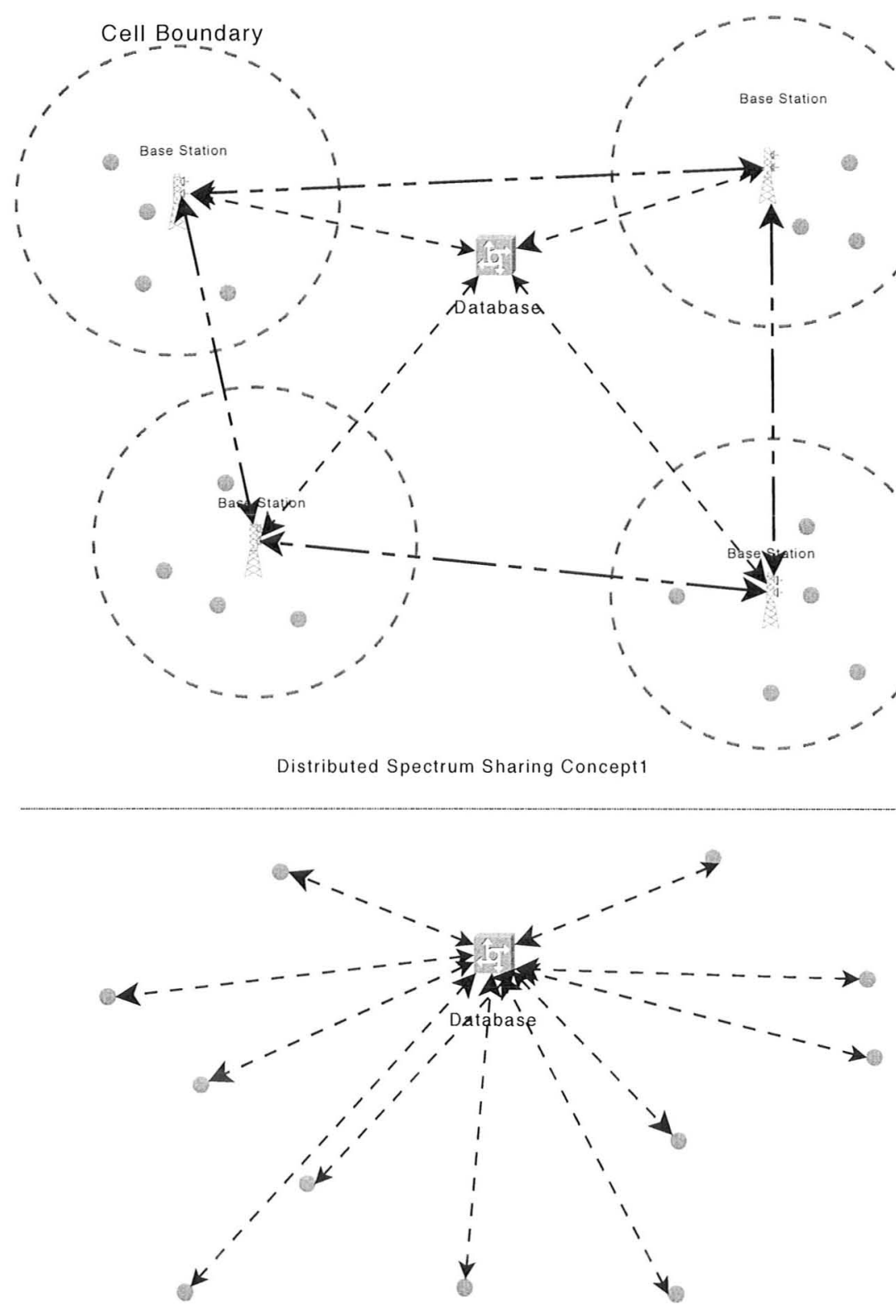

Distributed Spectrum Sharing Concept2

Figure 2.5: Distributed Spectrum Sharing Concept 
Figure 2.5 shows two conceptual distributed spectrum sharing scenarios. In the upper figure, CPEs in every cell are under the control of their corresponding base stations, and the base stations perform independently from each other. There may be cooperation among them, which is shown by dashed lines. This issue is discussed in the next section. A database may also be present, that stores the information about the available primary channels. Secondary base stations can access this information to better utilize the bands. In the bottom figure, another scenario is depicted, where secondary users are working individually and independently.

Another way of categorizing spectrum sharing schemes is based on the cooperation that secondary network entities have among themselves or with the primary network. In this respect, spectrum sharing can be cooperative, which means the secondary CPEs or base stations and primary ones are willing to share information regarding the medium or spectrum allocation, or non-cooperative, which consists of autonomous and independent users working in a greedy manner. The next section is devoted to discuss the pros and cons of these two schemes and the innovations proposed in the literature in this aspect.

\subsection{Cooperative Spectrum Sharing}

The concept of cooperation in spectrum sharing has a broad definition, but all cooperative secondary networks follow a common principle which obliges every spectrum allocation decision done by any network entity to not only consider the effect of the decision over the individual user, but also its effect on other secondary users or the overall network performance.

One way of modeling the interaction and decision making of such independent 
but cooperative users is to use cooperative game models [42]. In a cooperative game, before the players (secondary users) start the game (reusing the spectrum), a bargaining process is initiated. If an agreement, which is called a Nash Equilibrium, is reached in that process, it is mandatory for every player to follow it, otherwise players perform non-cooperatively. The keywords players, bargaining period and Nash Equilibrium are metaphors of network entities and their associated actions. A key important point is the existence of an equilibrium. A Nash Equilibrium is a set of decisions or strategies of all the players, where no player would benefit from changing its decision, assuming other players decisions remain the same. This equilibrium might be Pareto Optimal, but not necessarily[43]. The authors in [42] have modeled the secondary users as players and the channels as the bargaining goods. The users are assumed to be able to transmit over multi-channels. It is claimed that as the number of available channels increases, the number of Pareto Optimal points becomes larger, which increases the probability of achieving the optimal equilibrium in such a multi criterion optimization problem.

A problem that can arise is whether the sharing among the cooperative users is fair or not. The authors in [44] propose a cooperative game model where a number of primary and secondary users are the players using OFDM for modulation, and the objective is to find the transmitting power of each player in each of the frequency bands. The overall objective function is the summation of all the user powers and the optimization problem is formulated to minimize this function subject to QoS constraints of the primaries. In order to increase fairness amongst the players, the Nash Bargaining Solution (NBS) is proposed, which produces a fair and Pareto Optimal solution for the cooperative game[45]. This so-called "Fair Throughput Maximization 
Strategy" is achieved by satisfying a set of axioms [45] over the payoff function, which depends on the allocated user rates. The resulting optimization problem would be nonlinear. The authors have proposed using Sequential Quadratic Programming to find the solution. The proposed objective function in this paper is as below:

$$
\text { Minimize }_{p_{i, n}} \sum_{i=1}^{K} \sum_{n=1}^{N} p_{i, n}
$$

which is subject to QoS constraints both for primary and secondary users. In this formulation $p_{i, n}$ denotes the power of Player $i$ in Channel $n$. The axioms which convert the solution to be a fair one are as below in [46]:

* Pareto optimality of the solution(strong efficiency).

$\star$ Symmetry.

$\star$ Independence of irrelative alternatives.

$\star$ Invariance under change of location and scale (scale covariance).

For a two player game consisting of one primary and one secondary user, the converted fair problem would be:

$$
\operatorname{Maximize}\left(R_{1}-R_{p, \min }^{1}\right) \times\left(R_{s, \max }^{2}-R_{2}\right)
$$

The terms inside the objective are, the rate of the primary user, minimum rate for the primary user, maximum rate for secondary and secondary user rate, respectively. This objective would satisfy the above criteria for a fair solution to the cooperative game.

Another cooperative scenario is when the secondary network relays the data from the primary network and in return gains access to the spectrum owned by the primary. 
This approach is proposed in [47] with the use of a Stackelberg Game. In such games a player acts as the leader and other(s) as the follower(s). The authors have proposed this scenario: First the primary network (the leader) decides how to use the secondary nodes to relay the data and the price for each time unit which the secondary would be allowed to transmit. Afterwards, the secondary determines its spectrum access time share from each time frame, based on the previous decision taken by the primary. These decisions are made in secondary and primary APs, and the users of each network follow their corresponding APs. This two step decision is designed in a way to include fairness in the game, since if the primary increases the price for its own revenue maximization, the secondary won't be willing to buy those bands in its decision step. It is proved in this article that for this game there is a unique Nash Equilibrium with maximized utilities of both primary and secondary networks.

The idea of fair and efficient spectrum sharing among cooperative cognitive users is further investigated in [48]. In this article, primary and secondary users share the same spectrum in an underlay manner, meaning the spectrum usage is not exclusive. Primary users are protected from the excessive interference from the secondaries by introducing a constraint in the optimization problem. The objective function is the summation of secondary users utility functions as below:

$$
\max _{p=\left(p_{1}, \ldots, p_{N}\right)} \sum_{i=1}^{N} U_{i}\left(p_{1}, \ldots, P_{N}\right)
$$

Now it's the designer's choice as to how to define the utility function. As explained before, Nash axioms propose a number of conditions, and satisfying them would guarantee Pareto optimality and fairness of the solution. Thus it's one requirement 
forced over the utility function in this article. Using proportional fairness with Nash axioms ends in this objective function:

$$
\max _{p=\left(p_{1}, \ldots, p_{N}\right)} \sum_{i=1}^{N} \log \left(\gamma_{i}(P)-\gamma_{i, \min }\right),
$$

where $\gamma_{i}$ is the SINR of User $i . P$ is a set including users' transmit powers.

So far in this section, all the scenarios were cooperative where all the players were willing to come to an agreement before operating over the shared medium. In all the literature discussed in this section, the optimization problems included the utility functions of all the users, and were sometimes modified to generate a fair solution. In the next section a different scenario is investigated where users prefer to work in a non-cooperative manner.

\subsection{Non-cooperative Spectrum Sharing}

In the previous section, a scenario was discussed when the users were cooperating to reach a fair and optimal solution, before starting to operate over the shared bands. The objective functions in the previous scenario are usually the summation of the individual utility functions, and are defined in a way to guarantee well-known criteria of fairness and optimality. Here we discuss the opposite situation, where each secondary user tries to maximize its own utility function. The same discussion about the Nash equilibrium is applied here, but there is no guarantee that the resulting solution would be Pareto Optimal or fair.

The authors in [46] propose a non-cooperative approach to do the power allocation in the shared band. Each user in this MIMO-CDMA cellular network tries to minimize 
its own utility function which is defined as below:

$$
u\left(p_{1}, p_{2}, \ldots, p_{N}\right)=\left(\frac{p_{i}}{p_{\max }}\right)^{\alpha_{i}}\left(1-P_{\text {err }}\right)^{-\beta_{i}},
$$

where $p_{i}$ is the transmit power of $i^{\text {th }}$ user, $P_{\text {err }}$ its error rate. $\beta$ and $\alpha$ determine whether SINR has a higher priority, or error rate is more important. Since the users are acting selfishly, the only possible solution would be the Nash Equilibrium. Using the notation from [46], a power vector satisfies Nash Equilibrium if, $\forall i$ such that $1 \leq$ $i \leq N, \forall p:$

$$
u_{i}\left(p_{1}^{N E}, \ldots, p_{i-1}^{N E}, p_{i}^{N E}, p_{i+1}, \ldots, p_{N}^{N E}\right) \leq u_{i}\left(p_{1}^{N E}, \ldots, p_{i-1}^{N E}, p, p_{i+1}, \ldots, p_{N}^{N E}\right)
$$

$p$ is any strategy (transmit power set) that User $i$ can adopt other than the equilibrium one. The above inequality suggests that changing the equilibrium strategy isn't a wise decision, since no user will achieve any better performance, given other players strategies. This solution won't necessarily be Pareto Optimal, since it depends on the behavior of the users, and assuming they are acting rational, the solution might be Pareto Optimal, but not always fair. In order to guarantee a fair and Pareto Optimal solution, cooperative solutions should be applied, which were discussed in the previous section. In short, the conversion to a cooperative scenario scalarizes this multicriterion optimization problem and results in a combination of objective functions.

The authors in [49] describe a scenario, where multiple secondary users share the unused portion of a licensed spectrum in every time slot. It is assumed that the secondary network is aware of the unused portion of spectrum in every time slot. 
Payoff or objective function of Player $i$ is defined as:

$$
\pi_{i}\left(s_{1}, \ldots, s_{N}\right)=a\left(n_{i}\right) u_{i}\left(s_{i}, \eta_{U}\right)-b\left(n_{i}\right) C_{i}\left(s_{i}, \eta_{U}-s_{i}\right),
$$

where,

$$
\begin{aligned}
& C_{i}\left(s_{i}, \eta_{U}-s_{i}\right)=\left[s_{i}^{2}+\left(\eta_{U}-s_{i}\right)^{2}\right] \\
& u_{i}\left(s_{i}, \eta_{U}\right)=\left(\eta_{T}-\eta_{U}\right) \cdot s_{i} \\
& \eta_{U}=\sum_{i=1}^{N} s_{i}
\end{aligned}
$$

$s_{i}$ is the strategy of the $i^{t h}$ user, which is its transmit power. $\eta_{U}$ is the aggregate transmit power of users in the unused spectrum. $\eta_{T}$ represents the maximum tolerable interference. $C_{i}$ is the cost function and $u_{i}$ is the utility function that the user receives. The cost doesn't infer a monetary value, it just represents the resulting degradation of the performance due to the increased interference. $a\left(n_{i}\right), b\left(n_{i}\right)$ denote the weighting functions when the number of users is $n_{i}$. Every secondary user would try to maximize its own mentioned utility function, without considering its effect over other users or overall network performance. The authors have proved that this non-cooperative game, given the above objective functions will always have a Nash Equilibrium. An interesting observation in this paper is that the $\mathrm{NE}$ can be reached if every user can be aware of all other users' strategies, since the objective function of each user depends on the strategy of all the players. This would be problematic, since in a distributed manner, users just have local information and at most they may know about their neighborhood nodes. The Payoff-Enriched Adaptive Learning (PEAL) algorithm is proposed to help the game reach the equilibrium. In this approach users choose strategies based on local information, then gradually they adapt their strategies as they learn from the outcome of their last decisions. 
The authors in [50] take into consideration the energy consumption of the wireless terminals in a distributed non-cooperative CDMA network. As in previous scenarios in this section, the users take spectrum sharing decisions solely based on their own interests. Every user would have an objective function as below:

$$
u_{j}(p, c)=\frac{L R}{M p_{j}}\left(1-e^{-0.5 \gamma_{j}}\right)^{M},(\text { bits } / \text { Joule })
$$

where $M$ is the length of frame with header, and $L$ is its length without the header. $\left(1-e^{-0.5 \gamma_{j}}\right)^{M}$ is the probability of receiving error-free frames. $\gamma_{j}$ is the signal to interference ratio and is defined as

$$
\gamma_{j}=\frac{W}{R} \frac{h_{a, j} p_{j}}{\sum_{k \neq j, c_{k}=c} h_{a, k} p_{k}+\delta_{c}^{2}}
$$

In this equation, $W / R$ is the processing gain of CDMA. The interesting point in the utility function is the transmit power of user's being in the denominator. This would introduce the concept of battery life into the scope, since looking at the SINR function, it seems the more increase in transmit power, the more benefit the user gains, but introducing battery power would compromise this increase. It is proved that there exists a stable NE for every user, however SINR bounds might force the users to modify their NE decisions. In this case, in every time slot, each user based on the feedback from the previous decisions, adjusts its transmit power so that gradually it merges to either the NE or the bound SINR. 


\subsection{Pricing in Dynamic Spectrum Sharing}

The idea of spectrum pricing has emerged as an incentive to encourage the primary networks to let secondary carriers use their unused spectrum. This will also push the primary network to cooperate with the secondary network in better utilizing the shared spectrum. The prices are determined in various ways. If there is only one primary network, the network manager decides the prices based on the occupancy of its bands, the more occupied a band, the more expensive it would be for the secondary network. This pricing can be dynamic. For instance, assume the primary user to be a cell phone carrier. The geographical distribution of the network load would vary significantly in various portions of a day. This can be translated into time-varying or space-varying prices. In the case of multiple primary networks, the pricing can become a competition between the primaries. Since the secondary network can choose between the primaries, and the primary network that can offer a more reasonable price or better cooperation can gain more profit from its unused bands.

The authors in [51] investigate a scenario, where multiple primary networks compete to profit from selling their spectrum to a single secondary network. The spectrum demand of the secondary network determines its utility function. This utility function is formulated as below:

$$
U(\mathbf{b})=\sum_{i=1}^{N} b_{i} k_{i}^{(s)}-\frac{1}{2}\left(\sum_{i=1}^{N} b_{i}^{2}+2 v \sum_{i \neq j} b_{i} b_{j}\right)-\sum_{i=1}^{N} p_{i} b_{i}
$$

$b_{i}, k_{i}^{(s)}$ and $p_{i}$ are the amount of spectrum shared from Primary $i$, the spectral efficiency that secondary gains from using that spectrum and its price, respectively. For 
the primary service providers the cost and revenue functions are as

$$
R_{i}=c_{1} M_{i}, C\left(b_{i}\right)=c_{2} M_{i}\left(B_{i}^{r e q}-k_{i}^{(p)} \frac{W_{i}-b_{i}}{M_{i}}\right)^{2}
$$

$M_{i}$ is the number of connections of the primary network, $W_{i}$ indicates the size of spectrum and $B_{i}^{r e q}$ determines the required bandwidth for a primary connection. Based on these functions a Bertrand Game model is used, where primary users are players. The strategy of each player is the price that it decides for its band and the payoff function is the difference between revenue and cost functions. A Nash Equilibrium solution can be found by taking the derivative of profit functions and solving a set of linear equations. Due to the fact that in a distributed manner, a primary network can't be aware of the profit of all the other primaries, a dynamic distributed approach is introduced, where each primary's strategy gradually converges to the equilibrium.

The authors in [52] discuss a primary network that sells its excessive spectrum to secondary users. In this scheme, the primary QoS is guaranteed, but secondary traffic may be queued to give priority to primary traffic. Markov chains are applied to model the traffic, and depending on the state of the chain, the price is set. In order to introduce some sort of service guarantee for secondaries, the reliability of the waiting time is guaranteed to be $90 \%$, otherwise the price would be reduced to $20 \%$ of the actual optimized one. An acceptance function is defined as

$$
a\left(T_{90 \%}, p\right)=e^{-\alpha \cdot T_{90 \%}-\beta \cdot p-\gamma \cdot p \cdot T_{90 \%}},
$$

where $T_{90 \%}$ denotes the waiting time, $p$ is the price of the spectrum for secondary 
operation, and $\alpha, \beta$ are the delay and price sensitivities. This function indicates the probability that the secondary accepts the spectrum offer. The total gain of the primary operator from offering the spectrum in State $i$ would be

$$
U(p(i))=\pi_{i} \cdot v_{i, i+1} \cdot \frac{p_{i}}{\mu_{R U}}
$$

Then the optimization problem would be to maximize the following function.

$$
U(\mathbf{p})=\sum_{i=0, i \neq N}^{2 N} U(p(i)) .
$$

A different scenario is proposed in [53], where multiple secondary users are under the control of a base station. In this scheme pricing is used as an award to secondary users that use shorter paths. Secondaries can both use the base station to send their data to other secondaries or relay them through other existing secondaries. Since the second mode would increase the interference over the primary network, secondaries are rewarded if they use the first mode. The secondary users objective is to maximize their own profit and the base stations' is to maximize the network profit. Secondary cost and profit functions are given by

$$
\begin{gathered}
C_{n}\left(x_{n}\right)=a_{n} \cdot\left(U_{n}\left(x_{n}\right)+U_{n}\left(B_{n}-x_{n}\right)\right)-b_{n} \cdot U_{n}\left(B_{n}-x_{n}\right) \\
P_{n}\left(x_{n}\right)=U_{n}\left(x_{n}\right)+U_{n}\left(B_{n}-x_{n}\right)-C_{n}\left(x_{n}\right),
\end{gathered}
$$

where $U_{n}()$ denotes the utility function of Secondary User $n, x_{n}$ would be the amount of spectrum that it uses for type I communication, $B_{n}-x_{n}$ indicates the amount of spectrum used for type II and $a_{n}, b_{n}$ are the associated cost and reward values. The 
network profit function, which the base station is trying to maximize by setting the prices would be

$$
P_{B S}=\sum_{n \in N}\left[a_{n} \cdot\left(U_{n}\left(x_{n}\right)+U_{n}\left(B_{n}-x_{n}\right)\right)-b_{n} \cdot U_{n}\left(B_{n}-x_{n}\right)\right] .
$$

Since the base station objective function depends on the results gained from solving secondary optimization problems and vice versa, an iterative algorithm is designed where the secondary users at Iteration $k$ perform their optimization problem based on the prices at Step $k-1$, and the base station would set its optimized prices at Step $k$ based on the allocations requested by secondary users at Step $k-1$. 


\section{Chapter 3}

\section{Secondary Wireless Mesh Network Design Using Leased Frequency}

\section{Channels}

\subsection{Introduction}

Wireless mesh networks are currently being deployed as a cost-effective method of delivering high speed wireless Internet access. For example, mesh networks based upon the IEEE 802.11 air interface are now being standardized by the IEEE 802.11s task group, using unlicensed frequency spectrum. Unfortunately, these types of networks are often deployed in areas where the unlicensed bands suffer from excessive spectrum pollution. This may lead to unacceptable behavior due to uncontrollable co-channel interference from other spectrum users. This is especially an issue when the network offers real-time services which require reliable channel conditions.

An alternative to the unlicensed scenario is to use more tightly controlled channel 
sharing where a primary spectrum owner has excess available bandwidth. In this case the primary user may agree to lease these resources to the secondary mesh network operator. This type of sharing is already being considered. For example, a recent FCC directive has allowed the secondary use of frequency bands reserved for broadcast television, subject to spectrum owner interference constraints [2]. The availability of these channels may vary with geographic location and is determined by a spectrum broker [54] who sets the pricing of each channel at a given location. The use of this spectra is also subject to secondary user interference constraints so that the primary users are reasonably unaffected. Given the cost and interference constraints of the available spectra, a secondary network operator would like to design its network so that leasing costs are minimized while not exceeding its own channel interference constraints.

In this chapter we consider the practical deployment of secondary wireless mesh networks that use leased frequency spectra. The objective is to find the minimum cost placement of the secondary mesh nodes based on published primary network spectral leasing costs which may be a function of geographic location and time. The design is complicated by the fact that it must incorporate secondary node positioning, routing, and frequency allocation, subject to both primary and secondary network cumulative interference constraints.

The design problem is formulated as a mixed integer optimization that which gives the minimum cost of the secondary network deployment. Due to the complexity of the problem however, the optimum design is difficult to obtain except for small problem sizes. To accommodate more practical deployments, four heuristic algorithms are proposed and their performances are compared to solutions obtained from the 
optimization. The first algorithm is a greedy flow-based scheme (GFB) which iterates over the individual node flows based on solving a much simpler optimization at each step. The second one (MHFS) uses an iterated local search whose initial solution is based on constrained shortest hop routing performed on the flows separately. MHFSTS is similar to MHFS except the shortest path is performed for each time slot and each flow independently. A fast and efficient algorithm (Dijkstra-ILS) is proposed that finds the initial unconstrained routing using Dijkstra's shortest path algorithm, and using ILS makes the initial solution a feasible and locally improved result. Our results show that the proposed algorithms perform well for a variety of network scenarios.

\subsection{Related Work}

Secondary wireless mesh network design has been considered in Reference [55], where the secondary network is configured based on the availability of frequency channels belonging to a primary network. This work considers the design from a game theoretic viewpoint, involving competitions between multiple primary network owners and between different secondary users. This work however, does not consider the placement and frequency assignment for the secondary user base stations.

Reference [56] describes IP-based Service Overlay Networks (SONs) where the total network deployment cost is minimized by the proper placement of nodes and the appropriate acquisition of links from the primary owner.

A cooperative cognitive overlay mesh network design is proposed in [57] and [58]. In this work, it is assumed that the secondary network can be deployed over the infrastructure of the primary user, without adding any additional hardware to the 
existing nodes. A transmitter or a relay node in the primary network can assume the role of a secondary transmitter, since it has apriori information about the messages that are being transmitted. This scheme allows for network capacity maximization, since simultaneous packet transmission is possible in otherwise mutually interfering areas.

The work in [59] presents a mathematical formulation of the joint logical topology design, interface assignment, channel allocation, and routing in multi-channel wireless mesh networks. A local search algorithm was used to solve the optimization problem due to the high complexity involved in finding optimal solutions for large-scale networks. In [60], a scheme for interference management in WLAN mesh networks using free-space optical links was presented. A mechanism based on a genetic algorithm was proposed for managing both interference from internal network links and external interference.

Reference [61] discusses joint frequency assignment and routing in cognitive radio networks. An optimization is used to improve fairness in networks based on the IEEE 802.11 standard. In this work there is no primary network and pricing is not introduced in the formulation. The work in [62] is another that deals with joint physical, link and network layer optimization, in which user rate maximization is desired.

The problem dealt with in this thesis differs significantly from those discussed above. The proposed algorithms incorporate the published spectrum pricing, secondary node positioning, routing, and frequency allocation, subject to both primary and secondary network cumulative interference constraints. 


\subsection{Problem Formulation}

In the networks considered, we assume that the primary mesh network is fully deployed and has excess frequency spectrum available for use by a secondary user based on long-term leasing. It is assumed that the leasing costs are time-invariant and are available in advance of the secondary user network deployment. The costs of leasing spectrum will vary with geographic location and are expected to be a function of the scarcity of channels in the different regions. The use of any spectrum is also subject to published primary network cumulative interference constraints. User demands of the secondary network are specified by a multi-commodity bandwidth flow matrix which may be time-varying. We assume that the secondary user has specified a list of pre-determined candidate locations for its nodes.

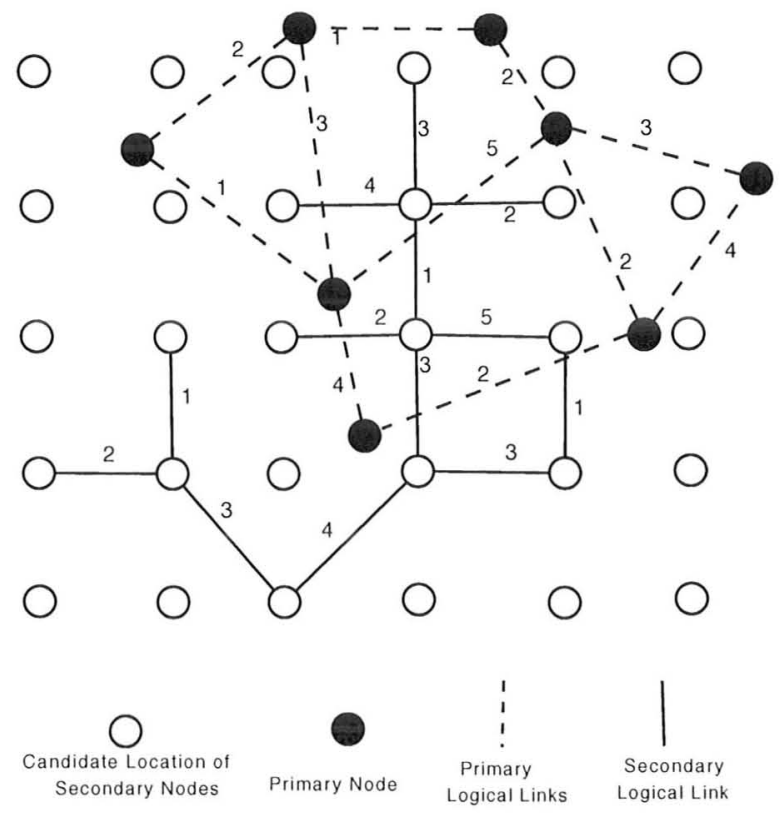

Figure 3.1: Primary And Secondary Networks 
The objective of the secondary network design is to minimize the network spectrum leasing costs by making an appropriate selection of frequency spectrum, node locations, and traffic routing. The formulation combines these selections under multiple primary and secondary network cumulative interference constraints. Figure 3.1 shows an example of a deployed secondary and primary network. The primary network consists of eight nodes, shown with round black circles, which communicate using frequency channels that define the links shown with dashed lines. The candidate secondary network node locations are shown as a set of 30 node positions, indicated by unshaded circles, arranged in a $5 \times 6$ grid with the selected links and the corresponding selected secondary nodes and frequency channels. In the following sections, we define an optimization which can be used to obtain the minimum secondary network deployment cost. We define $a_{m n, c}^{s d[t]}$ as a binary variable that represents the routing of the traffic from Source $s$ to Destination $d$ under the assumption of unsplittable flows at Time Slot $t$. Given a Directed Graph $G=(\mathcal{N}, \mathcal{L})$, where $\mathcal{L}$ is the set of physical secondary links and $\mathcal{N}$ is the set of candidate secondary nodes. $T$ denotes the set of time slots. There is a physical link between two nodes when they are close enough to communicate.

$$
a_{m n, c}^{s d[t]}=\left\{\begin{aligned}
1 & \text { if the traffic from } s \text { to } d \text { is routed through } \\
& \text { Link } l_{m, n} \text { from Nodes } m \text { to Node } n \text { on Channel } \\
& c \text { at Time Slot } t . m, n \in \mathcal{N}, t \in T \\
0 & \text { otherwise. }
\end{aligned}\right.
$$

We can write the total traffic carried by a Link $l_{m, n}$ on Channel $c$ at Time Slot $t$ 
represented by $\lambda_{m n}^{c[t]}$ as

$$
\lambda_{m n}^{c[t]}=\sum_{f_{(s, d)} \in \mathcal{F}}\left(a_{m n, c}^{s d[t]}+a_{n m, c}^{s d[t]}\right) \gamma^{s d[t]} . m, n \in \mathcal{N}, t \in T
$$

The left side of the above equation represents the aggregate traffic from Node $m$ to Node $n$ on Channel $c$ at Time Slot $t . \gamma^{s d[t]}$ is the expected traffic from Secondary Node $s$ to Secondary Node $d$ at that time slot.F $\mathcal{F}$ denotes the set of traffic flows, and $f_{(s, d)}$ indicates the flow from source $s$ to destination $d$. The total link aggregate traffic must not exceed the link capacity constraint, i.e.,

$$
\lambda_{m n}^{c[t]} \leq \Lambda \alpha_{m n}^{c} \forall l_{m, n} \in \mathcal{L}, c \in \mathcal{C}, t \in T
$$

$\Lambda$ represents the maximum acceptable link utilization level, while $\alpha_{m n}^{c}$ is the capacity of the link from Secondary Node $m$ to Secondary Node $n$ on Channel $c$. The following additional binary variables are also defined.

$$
\begin{aligned}
& x_{m n}^{c[t]}= \begin{cases}1 & \text { if there is a logical link between Secondary } \\
& \text { Nodes } m, n \text { on Channel } c \text { at Time Slot } t, \\
0 & \text { otherwise. }\end{cases} \\
& e_{m n}^{c}= \begin{cases}1 & \text { if there is a physical link between Secondary } \\
& \text { Nodes } m, n \text { on Channel } c, \\
0 & \text { otherwise. }\end{cases}
\end{aligned}
$$




$$
x p r_{u v}^{c[t]}= \begin{cases}1 & \text { if there is a logical link between Primary } \\ & \text { Nodes } u, v \text { on Channel } c \text { at Time Slot } t \\ 0 & \text { otherwise. }\end{cases}
$$

A logical link is a physical link that is selected for use. We now define,

$$
y_{m}^{i[t]}=1 \text { if } \exists n \in \mathcal{N}, l_{m, n} \in \mathcal{L} \text { such that }: x_{m n}^{i[t]}=1
$$

Therefore, the following constraint restricts the secondary nodes to have at most $I$ network interface cards at each time slot, i.e.,

$$
\sum_{c \in \mathcal{C}} y_{m}^{c[t]} \leq I, \quad \forall m \in \mathcal{N}, t \in T
$$

where $\mathcal{C}$ is the set of available channels, and $I$ is the maximum number of network interfaces. We can now write the flow conservation constraint, and assuming that $s, d, m, n$ are secondary nodes, the following equality should be satisfied $\forall f_{s, d} \in \mathcal{F}, t \in$ $T, m \in \mathcal{N}:$

$$
\sum_{n \in \mathcal{N}, l_{m, n} \in \mathcal{L}} \sum_{c \in \mathcal{C}} a_{m n, c}^{s d[t]} \gamma^{s d[t]}-\sum_{n \in \mathcal{N}, l_{m, n} \in \mathcal{L}} \sum_{i \in \mathcal{C}} a_{n m, c}^{s d[t]} \gamma^{s d[t]}= \begin{cases}\gamma^{s d[t]} & \text { if } s=m \\ -\gamma^{s d[t]} & \text { if } d=m \\ 0, & \text { otherwise }\end{cases}
$$

The secondary network design must be constrained so that it does not affect the operation of the primary network. We define $\beta_{(m, n),(o, p)}$ as the interference created between Secondary Links $l_{m, n}$ and $l_{o, p}$, when both are using the same frequency channel. This term will embed distance dependence that is pre-determined. We use $\beta \operatorname{pr}_{(u, v),(o, p)}$ to introduce the interference between primary and secondary links, where $\beta p r_{(u, v),(o, p)}$ is 
the interference between Primary Link $l_{u, v}$ and Secondary Link $l_{o, p}$. The interference constraint for secondary links is

$$
\begin{aligned}
& \left(\sum_{l_{o, p} \in \mathcal{L}, l_{o, p} \neq l_{m, n}} \beta_{(m, n),(o, p)} \times x_{o p}^{c[t]}\right) x_{m n}^{c[t]}+ \\
& \left(\sum_{u, v \in P} \beta p r_{(u, v),(m, n)} \times x p r_{u v}^{c[t]}\right) x_{m n}^{c[t]}+\mathcal{I}_{m, n}^{c[t]} \\
& \leq B x_{m n}^{c[t]}+\left(1-x_{m n}^{c[t]}\right) K \quad \forall l_{m, n} \in \mathcal{L}, \forall c \in \mathcal{C}, t \in T .
\end{aligned}
$$

In this equation, $P$ is the set of primary nodes and $\mathcal{N}$ are the secondary candidate nodes. $\mathcal{I}_{m, n}^{c[t]}$ introduces external interference on the Secondary Link $l_{m, n}$ operating on Frequency Channel $c$ at Time Slot $t . B$ is the maximum tolerable interference level over each secondary link. In order to avoid exceeding the tolerable interference on primary links, they are also included in the above summation. $K$ is defined as a large value, so that when $x_{m n}^{c[t]}=0$, the constraint is satisfied.

For primary nodes, we must also consider the fact that introduced interference from secondary nodes should not exceed a predetermined limit, i.e.,

$$
\begin{aligned}
& \left(\sum_{l_{o, p} \in \mathcal{L}} \beta p r_{(u, v),(o, p)} \times x_{o p}^{c[t]}\right) x p r_{u v}^{c[t]}+\mathcal{I} p_{u v}^{c[t]} \\
& \leq B x p r_{u v}^{c[t]}+\left(1-x p r_{u v}^{c[t]}\right) K p r \quad \forall u, v \in P, \forall c \in \mathcal{C}, t \in T
\end{aligned}
$$

where $\mathcal{I} p_{u, v}^{c[t]}$ is the external interference on Primary Link $l_{u, v}$ when operating on Channel $c \in \mathcal{C}$. $K p r$ is a large constant that makes the constraint be true, when $x p r_{u v}^{i[t]}$ is 
zero. We now define $X_{i}$ as the cost of deploying Node $i$, i.e.,

$$
X_{i}=\sum_{t \in T} \sum_{c \in \mathcal{C}} \sum_{n \in \mathcal{N}} x_{i n}^{c[t]} p_{i c}^{[t]}, \quad i \in \mathcal{N}
$$

where $p_{i c}^{[t]}$ is the price of Channel $c$ at Node $i$ in Time Slot $t$. We define $d_{i}$ as,

$$
d_{i}= \begin{cases}1 & \text { if Node } i \text { is deployed, } i \in \mathcal{N} \\ 0 & \text { otherwise }\end{cases}
$$

The following constraint ensures that the total number of nodes is less than a predetermined value $M$,

$$
\sum_{i \in \mathcal{N}} d_{i} \leq M
$$

The total cost, which is the objective function in the optimization problem can be written as,

$$
\text { TCost }=\sum_{i \in \mathcal{N}} X_{i}=\sum_{t \in T} \sum_{i \in \mathcal{N}} \sum_{c \in \mathcal{C}} \sum_{n \in \mathcal{N}} x_{i n}^{c[t]} p_{i c}^{[t]}+\sum_{i \in \mathcal{N}} d_{i}
$$

Our optimization problem is to find best candidates between physical links, node locations and routing so that the total cost of leasing frequencies is minimized while satisfying the constraints introduced above. In order to avoid deploying excessive nodes, the number of deployed nodes is added to the summation in the objective function. The complete problem can now be written as,

$$
\min _{d_{i}, x_{i m}^{c}} T \text { Cost }=\sum_{t \in T} \sum_{i \in \mathcal{N}} \sum_{c \in \mathcal{C}} \sum_{n \in \mathcal{N}} x_{i n}^{c[t]} p_{i c}^{[t]}+\sum_{i \in \mathcal{N}} d_{i}
$$


such that

$$
\begin{aligned}
& \lambda_{m n}^{c[t]}=\sum_{f_{(s, d)} \in \mathcal{F}}\left(a_{m n, c}^{s d[t]}+a_{n m, c}^{s d[t]}\right) \gamma^{s d[t]} \\
& \lambda_{m n}^{c[t]} \leq \Lambda \alpha_{m n}^{c} \\
& \sum_{c \in \mathcal{C}} y_{m}^{c[t]} \leq I, \forall m \in \mathcal{N}, t \in T \\
& \left(\sum_{l_{o, p} \in \mathcal{L}, l_{o, p} \neq l_{m, n}} \beta_{(m, n),(o, p)} \times x_{o p}^{c[t]}\right) x_{m n}^{c[t]}+\left(\sum_{u, v \in P} \beta p r_{(u, v),(m, n)} \times x p r_{u v}^{c[t]}\right) x_{m n}^{c[t]}+ \\
& \mathcal{I}_{m, n}^{c[t]} \leq B x_{m n}^{c[t]}+\left(1-x_{m n}^{c[t]}\right) K \quad \forall l_{m, n} \in \mathcal{L}, \\
& \forall c \in \mathcal{C}, t \in T \\
& \left(\sum_{l_{o, p} \in \mathcal{L}} \beta p r_{(u, v),(o, p)} \times x_{o p}^{c[t]}\right) x p r_{u v}^{c[t]}+\mathcal{I} p_{u, v}^{c[t]} \leq B p r_{u v}^{c[t]}+\left(1-x p r_{u v}^{c[t]}\right) K p r \\
& \forall u, v \in P, \forall c \in \mathcal{C}, t \in T \\
& \sum_{i \in \mathcal{N}} d_{i} \leq M
\end{aligned}
$$

AMPL/CPLEX is used in order to find the optimal solution as well as the heuristic solutions of the above problem. In order to do that, the problem should be converted to a linear mixed integer optimization problem. The only nonlinear section of this problem is the secondary interference constraint in Equation 3.3. To be more specific, 
the following term that constitutes of variable multiplications:

$$
\left(\sum_{l_{o, p} \in \mathcal{L}, l_{o, p} \neq l_{m, n}} \beta_{(m, n),(o, p)} \times x_{o p}^{c[t]}\right) x_{m n}^{c[t]},
$$

where $x_{o p}^{c[t]}, x_{m n}^{c[t]}$ are both variables. A method is proposed in [63] to convert these binary multiplications into multiple linear inequaties. In this method a temporary variable is defined and is set equal to the troublemaking terms using linear inequalities. The temporary positive integer variable used here is IntTemp $p_{m n}^{c[t]}$ and is computed as below:

$$
\begin{aligned}
& \text { IntTemp } \leq 10_{m n}^{c} \times x_{m n}^{c[t]} \\
& \operatorname{IntTemp} p_{m n}^{c[t]} \leq \sum_{l_{o, p} \in \mathcal{L}, l_{o, p} \neq l_{m, n}} \beta_{(m, n),(o, p)} \times x_{o p}^{c[t]} \\
&\left(\sum_{l_{o, p} \in \mathcal{L}, l_{o, p} \neq l_{m, n}} \beta_{(m, n),(o, p)} \times x_{o p}^{c[t]}\right)-10^{5} \times\left(1-x_{m n}^{c[t]}\right) \leq \operatorname{IntTemp} p_{m n}^{c[t]}
\end{aligned}
$$

This problem is very complex and can easily be shown to be NP-complete, as it includes graph coloring as a special case. An approach for solving these problems is the Branch and Bound method [64], however, to accommodate reasonable problem sizes, it is necessary to consider suboptimal methods. In the following section, we discuss four different heuristic methods to tackle this problem. The performance of these algorithms are compared in Chapter 4.1 and are also compared with the optimum result in some small network design situations. 


\subsection{Proposed Algorithms}

In order to reduce the size of the problem, we try to break it down into a number of smaller problems with more limited search space. One way to do this is to do the problem on the flows separately. For example, assume the problem should be solved for Flows A and B . First we solve the problem assuming only Flow A exists. The size of this problem would be considerably smaller than the original one, since only one flow should be routed. After that, we assume the routing, node placement, and frequency assignment of Flow 1 to be fixed, then we perform the optimization problem assuming just Flow B. In this second step, not only one flow should be routed, but also the search space would be more limited due to the fixed variables. This approach is referred to as the Greedy Flow-Based (GFB) Algorithm and is summarized in Algorithm 1. Since the optimization problem in each step can't see the future flows, it might select a path that may be far from the optimal solution. Another problem that might occur is that the result would generate multiple bottlenecks in the routing. When a link is selected in one step, the optimization problem tries to route as much traffic as possible over that link so that the price would be minimized. This would also increase the number of hops and consequently a higher delay. Obviously much fewer iterations are required to generate the suboptimal result, since in each step one flow is being routed and considering the fixed variables, a smaller size problem is solve in each step.

A different approach for finding suboptimal solutions in large scale optimization problems is the iterated local search (ILS) technique [59][45]. An algorithm based on this is shown in Algorithm 2. We start with an initial solution to the problem. Then for every two secondary nodes in each time slot, if there is a logical link in 
use between them, we perform the optimization problem from Section 3.3 assuming that the network remains fixed except for those two nodes and every link connected to them. If the new solution improves over the previous one, then we update the topology and the new set of logical links. This local search is done for all logical links.

The proposed method is very promising, since it is a local search and can be used for dynamic cases when there is a local change in the network. The problem that arises here is how to find a decent initial solution. Since the local search improves the results locally, thus highly depends on the initial configuration. If the initial starting point of ILS is not appropriate in the sense of routing and appointed frequencies, the final solution cannot be expected to be close to the optimal result. A promising starting point is the one found by using shortest path routing algorithms. In this case a smaller optimization problem is solved first to find the constrained shortest path routing, without considering the frequency prices. After this step, ILS is performed to modify the assigned frequencies. In other words the problem is split into minimum hop routing and local minimum pricing. In order to find the shortest paths, we set the objective function of problem in Section 3.3 to the summation of the number of hops. The pseudocode of ILS is shown in Algorithm 2. Two different heuristics are proposed based on these arguments. 

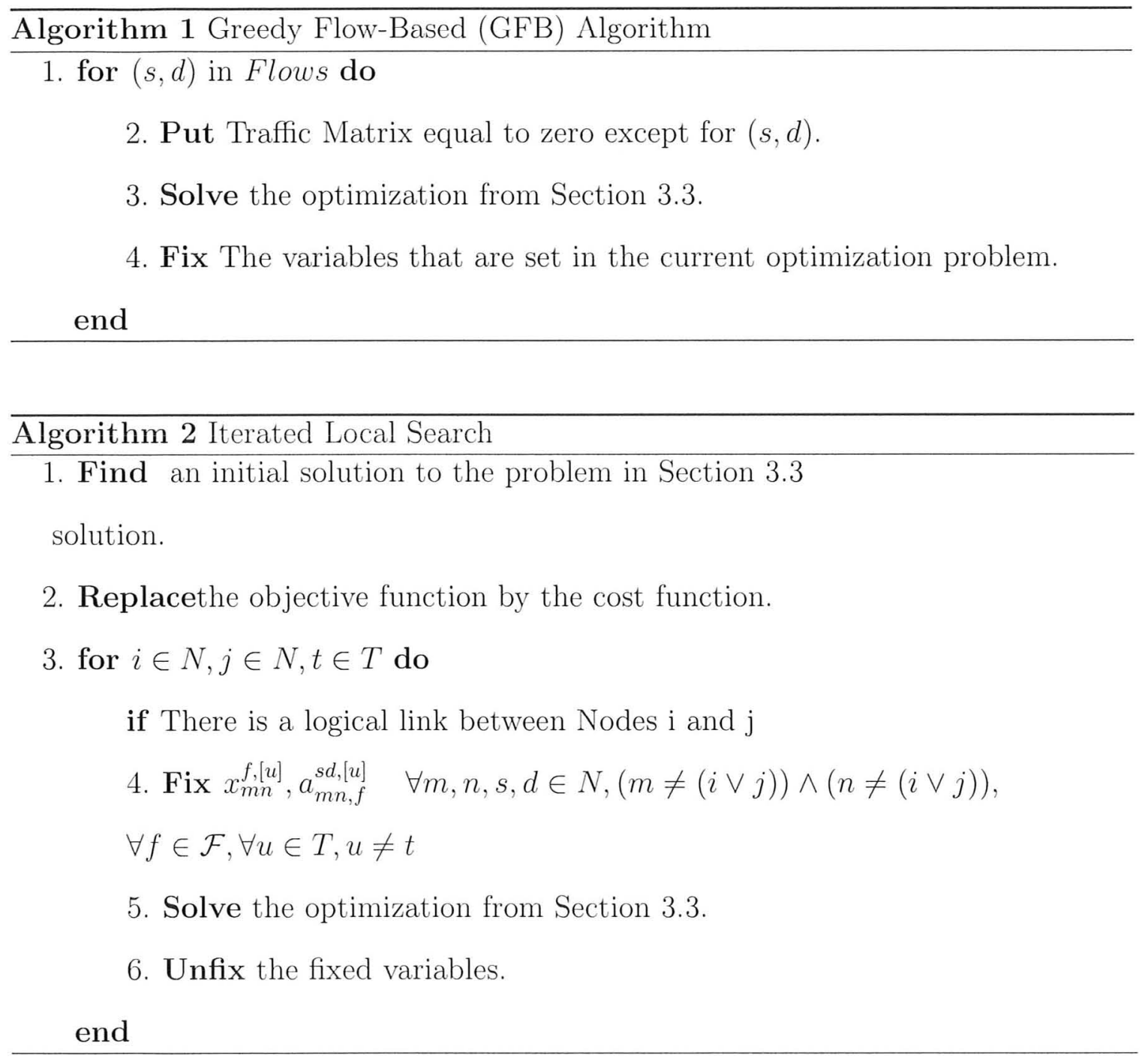

The first algorithm based on ILS is called Minimum Hops Flows Separately(MHFS). It performs first by finding the constrained shortest path for flows separately, similar to GFB without considering the pricing. This initial solution is then improved by performing the ILS. The pseudocode is shown in Algorithm 3.

The other ILS-based algorithm is the same as MHFS, except the initial solution is found by doing the constraint shortest path for each flow and in every time slot separately from both other time slots as well as other flows. So for example if two 
flows should be routed in three time slots, six smaller optimization problems are to be solved. This would greatly reduce the size of problem in each step. This algorithm is named Minimum Hops Flows Separately Time Slots Separately (MHFSTS). Algorithm 4 better clarifies the steps taken to perform this heuristic.

In the all three algorithms discussed so far the computation complexity would still be $O(\exp )$, since the same problem with a different objective is solved. The only computation advantage is the search space, which is highly limited in each step, as well as doing the routing and frequency assignment separately. But there is no guarantee that the computation size will not explode with the increase in the size of the problem. This problem can be solved by finding the initial solution to ILS using Dijktra's shortest path algorithm, which is $O\left(N^{2}\right)[65]$. Since ILS solves a problem with a limited search space in every step, the overall complexity would still be $O\left(N^{2}\right)$. However, the original Dijkstra should be modified due to having multiple traffic flows in multiple time slots.

The only constraint that is satisfied in the results received from the modified Dijkstra, in addition to flow conservation, is the link capacity. Other constraints are satisfied by performing ILS. Thus contrary to the previous heuristics, where the initial solution to ILS step was a feasible solution, the one used here is not necessarily feasible. So ILS does the frequency assignment both for reducing the cost and producing a feasible solution. Thus relaxations are required in ILS. First the routing for every flow in each time slot is found using Dijkstra, after each flow is routed, the path cost matrix of Dijkstra is updated to make sure no link is overloaded, and in the case the routed traffic on a link is exceeded, the cost of the link is increased to indicate a more expensive channel would be available. For example assume the cost of the 
path between Nodes 1 and 2 is 20, meaning Channel 1 is already fully occupied and Channel 2 is available. Now if the routed traffic over this link exceeds the available capacity it's cost will be increased by 10, meaning Channel 2 is fully occupied between Nodes 1,2 and the available channel is at frequency 3. Performing this modification in Dijkstra's shortest path algorithm would avoid routing traffic over links that their capacity is reached. Therefore the final results from the Dijkstra algorithm not only would be a feasible routing of the traffic flows, but also has a frequency allocation which partly satisfies the link capacity constraint. This allocation is then further modified in the following ILS.

In the previous heuristics, ILS was performed over a feasible initial solution. So in every step all the constraints were satisfied. But in the Dijkstra-ILS method, since the initial solution is not feasible, ILS gradually makes a feasible solution. In order to do this, all the constraints are checked just for the first node, except flow conservation that is satisfied globally, when ILS kicks off from the first node. Then when it goes to the second node, the set of nodes that are check for consistency with constraints includes Nodes 1 and 2. Eventually all the nodes would be included in this set, when ILS is done. Algorithm 5 shows the pseudocode for this heuristic. The search space for ILS is limited to just one node and the links connected to it in every step. 


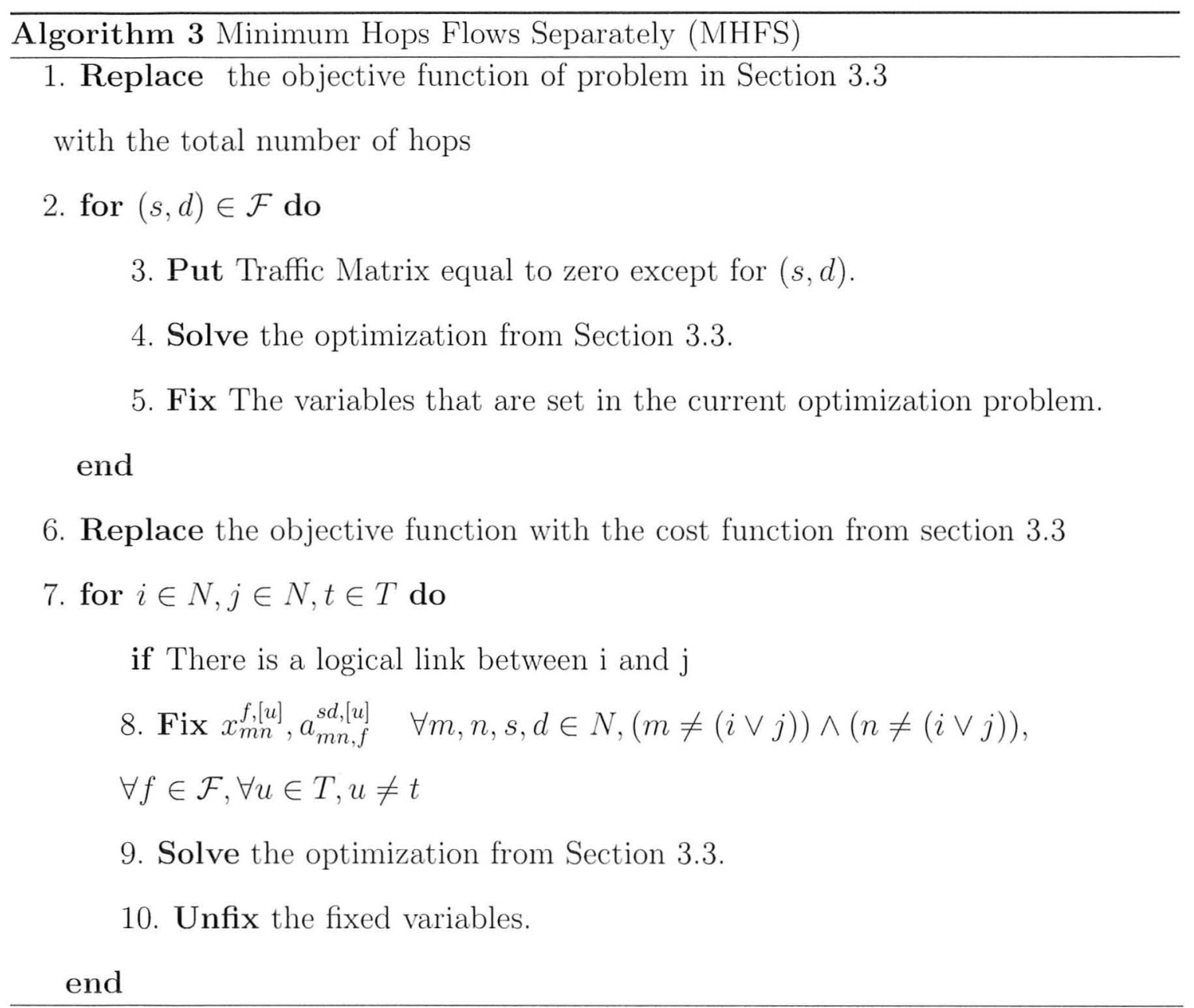




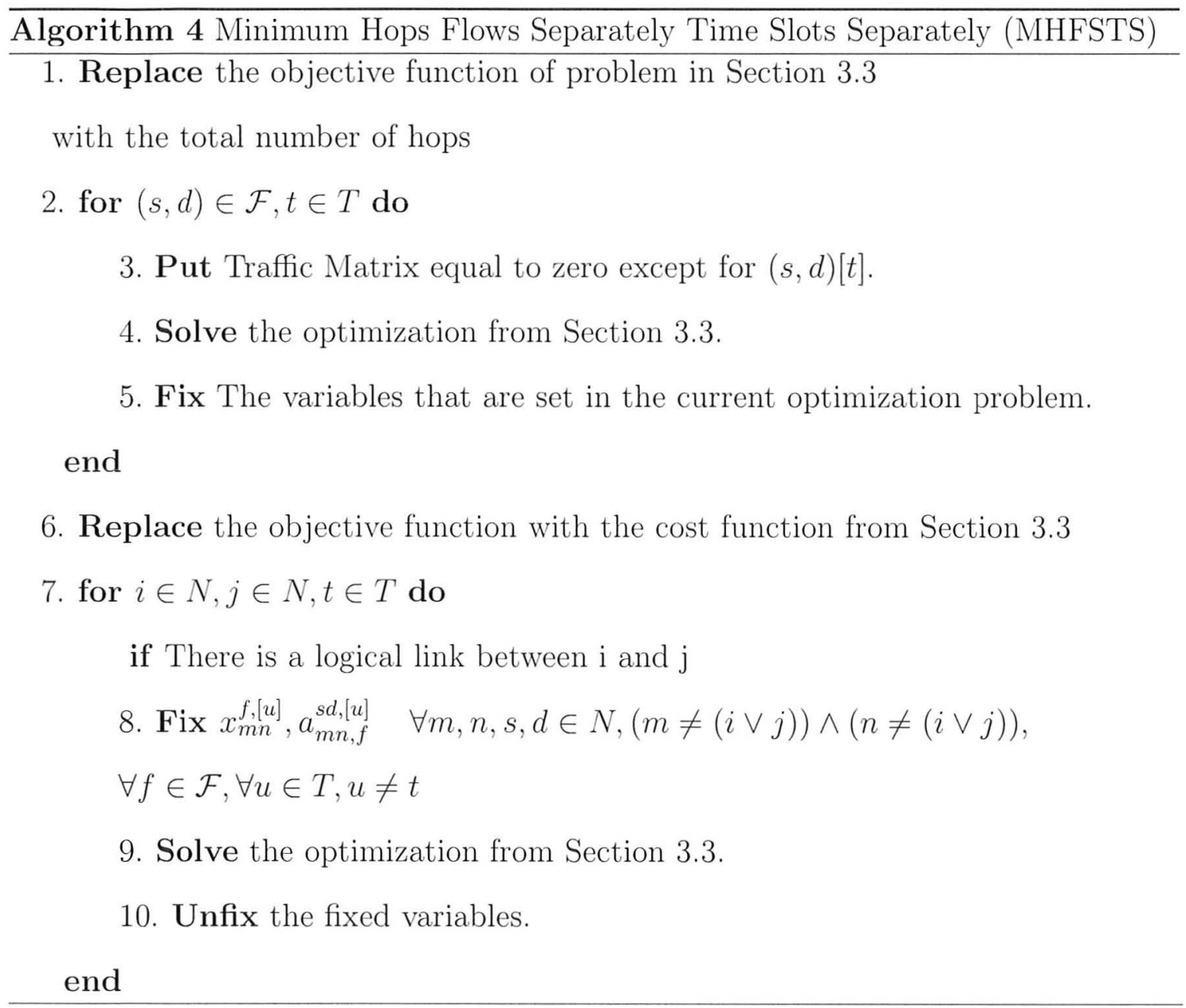




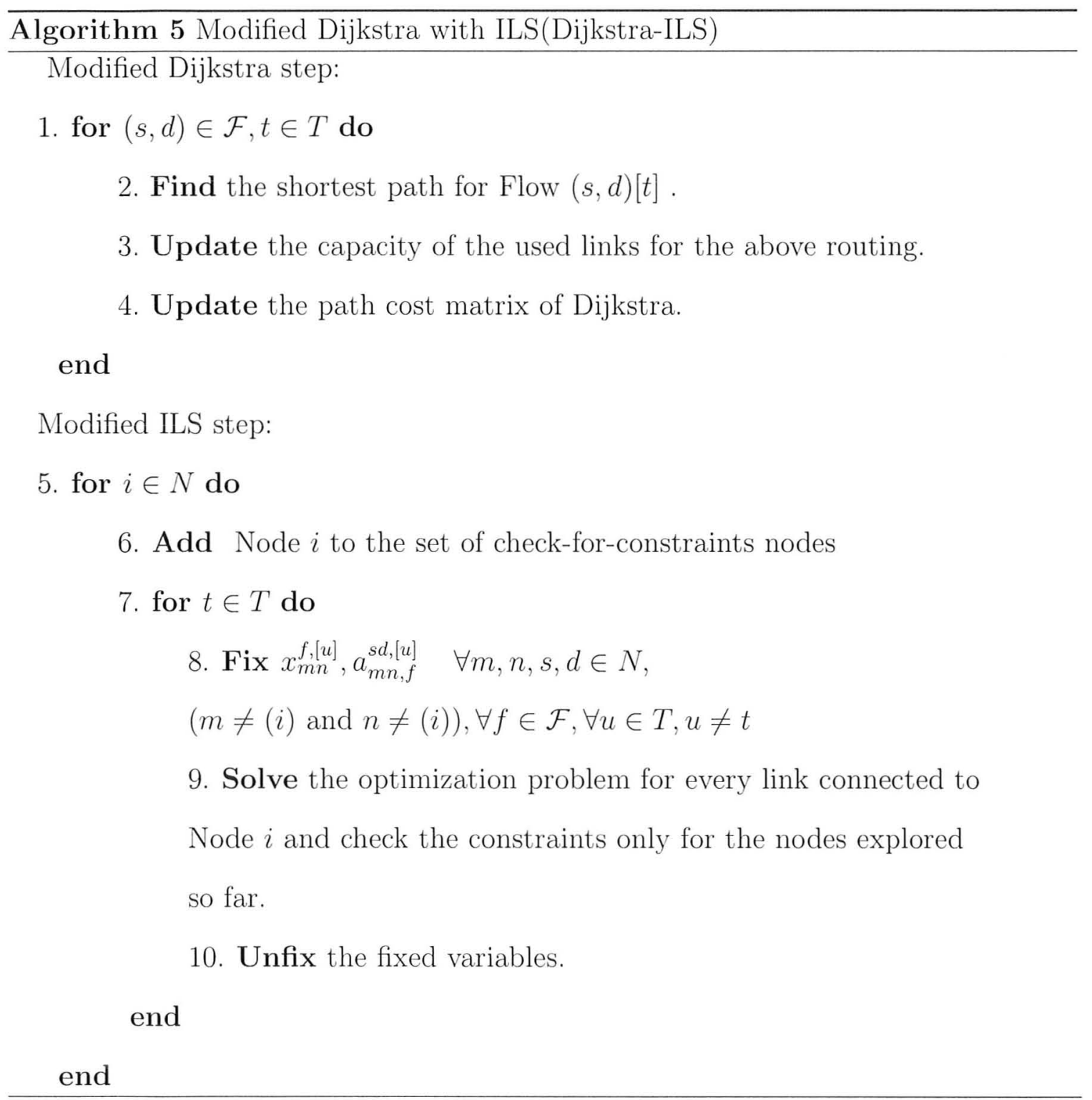




\section{Chapter 4}

\section{Results}

\subsection{Primary Network Topologies and Parameters: Static Scenario}

First we start with the static scenario, where none of the parameters or variables are time varying. Thus solving the problem just in one time slot would suffice. We assume a primary network with 9 nodes operating over five frequency channels as shown in Figure 4.1 with dotted lines and black circles. Secondary candidate nodes are assumed to be spread uniformly over a rectangular area, as shown by white circles. The traffic matrix is randomly generated for different source and destination pairs and also for different numbers of flows. The interference threshold is set in a way to prevent overlapping links in both primary and secondary networks, and the assumed normalized prices are as as follows, 10 price units for F1, 20 for F2, 30 for F3, 40 for F4 and 50 for F5. We have assumed that different regions have the same frequency prices. In this example we assume that the traffic flows are fixed. 
Figure 4.1 depicts a typical secondary network deployment and routing when three traffic flows were assigned in the secondary network with 20 candidate places for nodes. Solid lines depict the selected secondary links, and the chosen frequency is also shown for each link. As it can be seen in the figure, the most heavily used frequencies are F1 and F2, and it is clear that the secondary network has avoided the congested section of the primary network. This is obviously required to meet the interference constraint of the primary network. The total normalized cost in this deployment is 300 .

In the next example we investigate the effect of changing link utilization, and the number of flows for GFB and MHFS algorithms. In this case, 30 candidate secondary nodes are assumed with the same configuration as discussed in the previous example. The maximum number of NICs is set to 6 , and the link capacity is equal to 3 units for all secondary links. It was also assumed that at most, 15 secondary base stations can be deployed. The other assumptions are the same as in the previous example.

The overall cost for various numbers of flows and link utilizations are shown in Tables 4.1 to 4.3. Because of the high computational load of finding the optimal results and also the limited available resources, the optimal results are found for less than four traffic flows. 

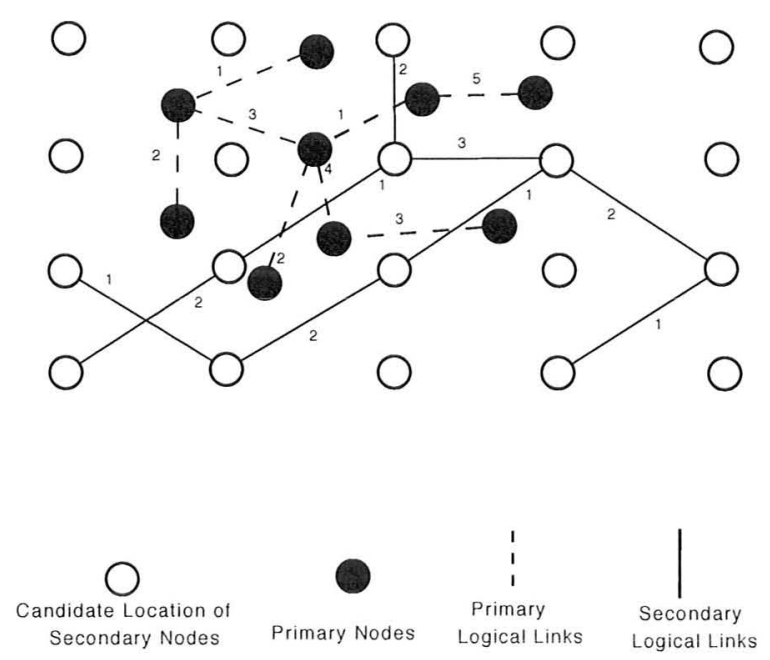

Figure 4.1: Secondary Network Deployment Over Primary Network

\subsection{Tables and Comparisons}

Table 4.1 MHFS Overall Cost
\begin{tabular}{|c|ccccccccc|}
\hline Utilization & .3 & .4 & .5 & .6 & .7 & .8 & .9 & 1 \\
\hline Flows & & & & & & & & \\
1 & 40 & 40 & 40 & 40 & 40 & 40 & 40 & 40 \\
2 & 160 & 160 & 160 & 170 & 170 & 170 & 140 & 140 \\
3 & 380 & 380 & 320 & 200 & 200 & 210 & 200 & 200 \\
4 & 420 & 310 & 290 & 290 & 300 & 290 & 300 & 310 \\
5 & 540 & 350 & 350 & 320 & 320 & 330 & 330 & 310 \\
6 & 630 & 490 & 410 & 360 & 400 & 370 & 400 & 400 \\
7 & 680 & 490 & 480 & 530 & 510 & 530 & 490 & 490 \\
8 & 1330 & 680 & 640 & 480 & 480 & 450 & 470 & 470 \\
9 & 930 & 640 & 660 & 560 & 500 & 540 & 540 & 550 \\
10 & 1120 & 740 & 650 & 660 & 670 & 610 & 630 & 550 \\
Avg & 603 & 428 & 400 & 361 & 359 & 354 & 354 & 346 \\
\hline
\end{tabular}


Table 4.2 Greedy Flow-Based Overall Cost

\begin{tabular}{|c|cccccccc|}
\hline Utilization & .3 & .4 & .5 & .6 & .7 & .8 & .9 & 1 \\
\hline Flows & & & & & & & & \\
1 & 40 & 40 & 40 & 40 & 40 & 40 & 40 & 40 \\
2 & 140 & 140 & 140 & 140 & 140 & 140 & 140 & 140 \\
3 & 280 & 290 & 180 & 180 & 220 & 220 & 190 & 190 \\
4 & 380 & 310 & 260 & 250 & 220 & 300 & 230 & 220 \\
5 & 400 & 300 & 300 & 390 & 270 & 310 & 260 & 290 \\
6 & 630 & 450 & 330 & 380 & 360 & 320 & 810 & 420 \\
7 & 680 & 470 & 460 & 460 & 490 & 390 & 330 & 330 \\
8 & 830 & 610 & 730 & 530 & 480 & 490 & 540 & 380 \\
9 & 840 & 660 & 600 & 580 & 590 & 510 & 450 & 470 \\
10 & 970 & 740 & 690 & 690 & 640 & 600 & 510 & 500 \\
Avg & 519 & 401 & 373 & 364 & 327 & 345 & 350 & 298 \\
\hline
\end{tabular}

Table 4.3 Optimal Overall Cost

\begin{tabular}{|c|cccccccc|}
\hline Utilization & .3 & .4 & .5 & .6 & .7 & .8 & .9 & 1 \\
\hline Flows & & & & & & & & \\
1 & 40 & 40 & 40 & 40 & 40 & 40 & 40 & 40 \\
2 & 140 & 140 & 140 & 140 & 140 & 140 & 140 & 140 \\
3 & 260 & 260 & 180 & 180 & 180 & 180 & 180 & 180 \\
Avg & 146.6 & 146.6 & 120 & 120 & 120 & 120 & 120 & 120 \\
\hline
\end{tabular}

\begin{tabular}{l}
\hline Table 4.4 GFB and MHFS Comparison for Varying Network Sizes \\
$\qquad$\begin{tabular}{|c|cc|}
\hline Network Size & GFB & MHFS \\
\hline 10 & 197.14 & 211.4 \\
15 & 330 & 322.8 \\
20 & 471.4 & 445.7 \\
25 & 462.8 & 420 \\
30 & 528.5 & 517.1 \\
35 & 582.8 & 657.1 \\
40 & 640 & 474.3 \\
45 & 611.4 & 594.3 \\
50 & 642.8 & 617.1 \\
\hline
\end{tabular}
\end{tabular}

In all of the results that we have considered, both algorithms do a reasonable job of obtaining low cost solutions. Comparing the average results in Tables 4.1 and 4.2 
shows that GFB generally outperforms MHFS. This can be justified by considering the fact that GFB tries to minimize the cost at every iteration, but MHFS starts with shortest paths for flows, and then tries to minimize the cost. The shortest path initial solution does not always provide a good starting point. Thus it is reasonable to expect more expensive results from MHFS in general, but as can be seen from Tables 4.1 and 4.2 , there are several instances where MHFS has outperformed GFB, i.e, when link utilization is 0.6 for 5, 6, 8, 9 and 10 traffic flows. This is because in GFB, when a path is chosen for a flow, it may lead to excessive costs for future routed flows. This happens since routing and placement is done for flows consecutively without considering their mutual effects. The same argument can be applied for MHFS, but in this case routing and frequency assignment are done separately, and there is more freedom. Thus GFB has on average a lower overall deployment cost, but it does not always outperform MHFS.

The effect of changing link utilization is also investigated in Tables 4.1 and 4.3. As can be seen from the average values, decreasing link utilization increases the overall cost of the secondary network deployment, since the secondary network owner must pay for more links. A contradicting point is that sometimes decreasing link utilization has decreased the network price. For example, in GFB, when the number of flows is set to 8 , decreasing the link utilization from 0.5 to 0.4 has reduced the cost from 730 to 610. Similarly, in MHFS with 9 traffic flows, decreasing the utilization from 0.8 to 0.7 has resulted in a cost decrease from 540 to 500 . This is because both of these algorithms do the flow optimization separately, thus they do not have any information about the remaining non-routed traffic. Sometimes a lower link utilization makes the optimizer choose a different path for a specific flow, which results in less 
expensive paths for the remaining traffic flows, and thus the overall network cost may be reduced. But again, the average values show that in most of the cases, the decrease in link capacity has resulted in the increase of the secondary network's overall cost.

From a computational load point of view, both algorithms require much less time than the optimal solution and we are able to solve very large problems. For example, when finding the optimal solution for three flows, optimal results required 10 million or more iterations on average, but this figure in the proposed algorithms was less than 100,000. However, MHFS is faster than GFB, i.e., the higher the number of iterations results in better solutions on average. MHFS search is more suitable when there is a local change in the network. In that case, MHFS may be able to properly address the change.

To further explore the algorithms we also investigate the effect of increasing the secondary network size. We fixed the number of flows to six, link utilization equal to 0.5 , link capacity equal to three and maximum NICs set to 6 . Then the number of secondary node candidates was changed from 10 to 50 . Traffic flows are generated randomly and the average values are shown in Table 4.4. These results show that GFB again generates lower cost results than MHFS, which happens for the reasons discussed previously.

In the next sections, dynamic scenarios and various topologies are explored. It is assumed that the traffic flows vary in each time slot. Due to the computation complexity of the problem, optimization problems are solved for three time slots. Secondary candidate nodes are spread uniformly in a $6 \times 5$ rectangle. Traffic flows are generated randomly and the routing is assumed to be connection oriented, meaning a specific flow is routed only over one path. 


\subsection{Primary Network Topologies and Parameters: Dynamic Scenario}

The first topology to be explored in the dynamic scenario consists of a fully connect primary network of six nodes, as shown in Figure 4.7. Ten frequencies are assumed to be shared between both primary network and the secondary, while Frequency Channels 7-10 are being exclusively available for the secondary network with a higher price. Prices range from 10-100 for Frequencies 1-10 respectively. The problem is solved for traffic flows ranging for 1 flow to 12 flows, and the link utilization from 0.3 -1. Link capacity is assumed to be 3 units of traffic. The maximum traffic amount of each flow is restricted at 0.9 unit. Maximum allowable number of deployed nodes is set to 25. Four proposed heuristics are compared and the results are shown in the tables. For each heuristic three tables are included: One for the overall cost of assigned frequencies, one for the number of hops and the last one for the number of deployed nodes.

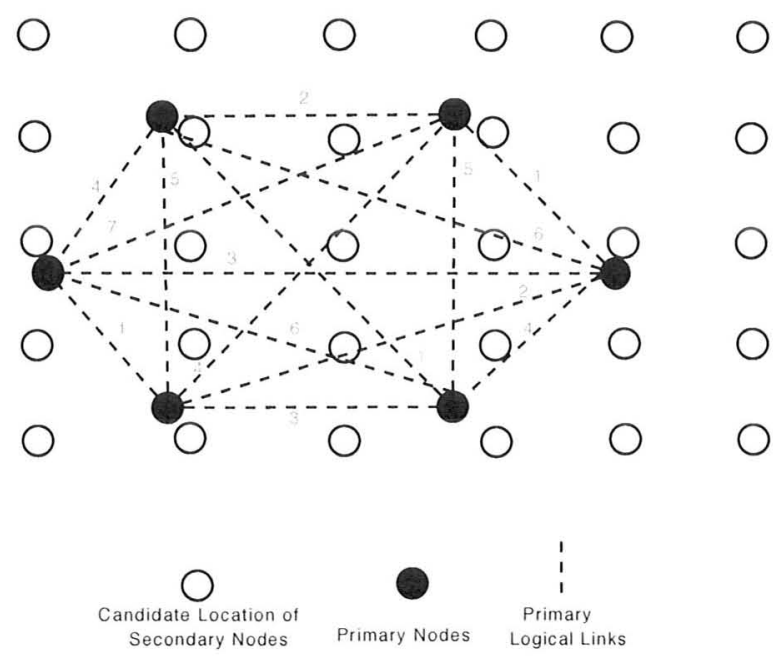

Figure 4.2: Secondary Network Deployment Over Primary Network 
The second topology to be explored in the dynamic scenario has a primary network as shown in Figure 4.3. Six frequencies are assumed to be shared between both primary network and the secondary one, with the price ranging from 10-60 for Frequencies 1-6 respectively. Frequency Channel 6 is assumed to be exclusively used by the secondary network. The remaining parameters are the same as the ones used in the fully connected topology.

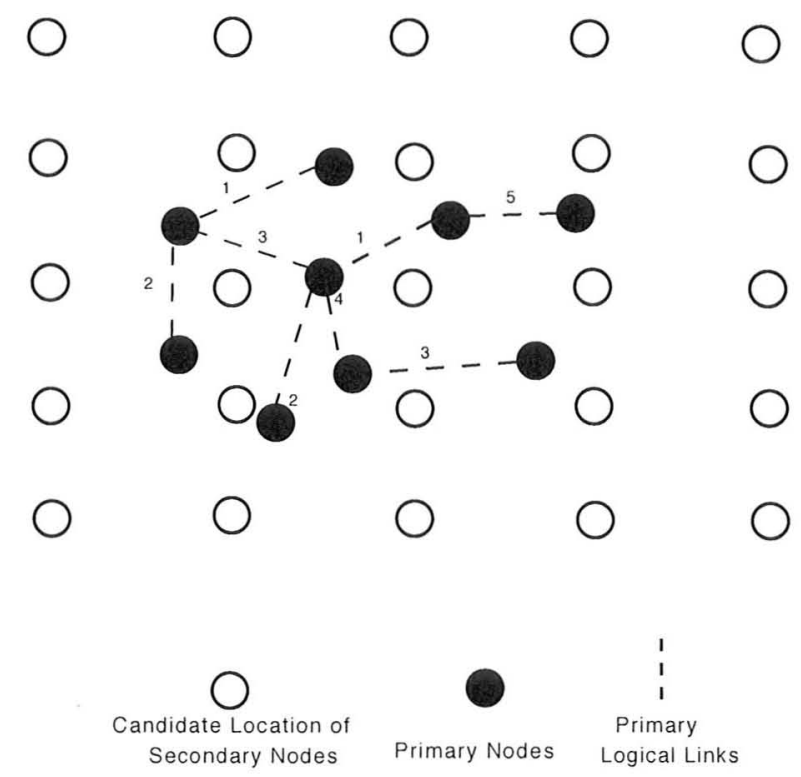

Figure 4.3: Secondary Candidate Nodes Over Primary Network In Random Mesh Topology 


\subsection{Tables and Comparisons}

\subsubsection{Overall Cost Tables}

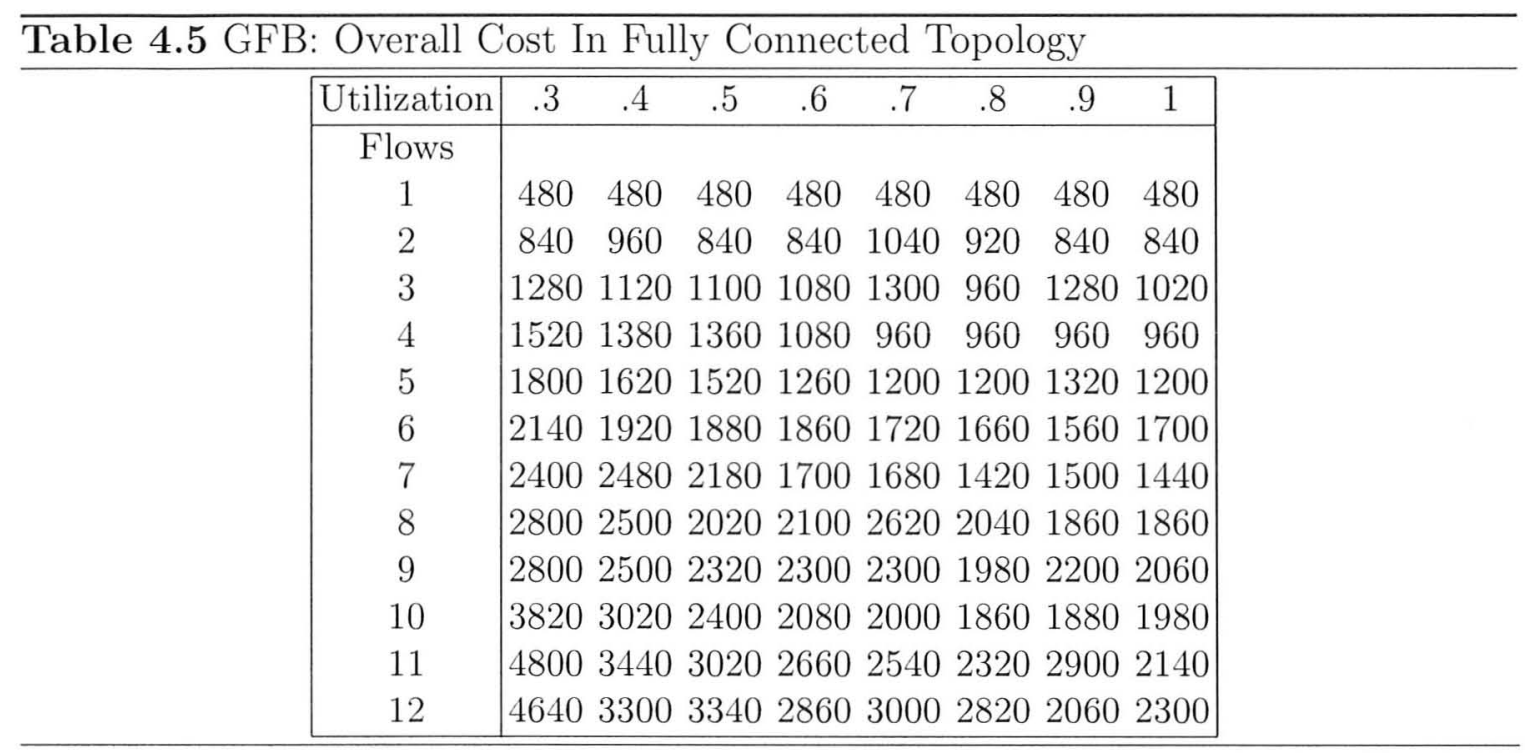

Table 4.6 GFB: Overall Cost In Random Topology

\begin{tabular}{|c|c|ccccccccc|}
\hline Utilization & .3 & .4 & .5 & .6 & .7 & .8 & .9 & 1 \\
\hline Flows & & & & & & & & & \\
1 & 420 & 420 & 420 & 420 & 420 & 420 & 420 & 420 \\
2 & 860 & 800 & 820 & 780 & 780 & 860 & 860 & 860 \\
3 & 1100 & 1100 & 1100 & 900 & 1060 & 1060 & 780 & 910 \\
4 & 1200 & 1340 & 1260 & 1360 & 1180 & 960 & 960 & 960 \\
5 & 1560 & 1460 & 1220 & 1340 & 1480 & 1560 & 900 & 1140 \\
6 & 2140 & 1860 & 1620 & 1440 & 1480 & 1760 & 1120 & 1080 \\
7 & 1980 & 1980 & 1920 & 1600 & 1600 & 1800 & 1500 & 1500 \\
8 & 2920 & 2360 & 2300 & 1840 & 1560 & 1980 & 1600 & 1920 \\
9 & 2160 & 2500 & 2580 & 2000 & 1960 & 1600 & 2060 & 1540 \\
10 & 2760 & 2680 & 2540 & 2180 & 2220 & 2220 & 1780 & 1740 \\
11 & 3840 & 3480 & 2960 & 2700 & 2440 & 2500 & 2200 & 2280 \\
12 & 3960 & 3480 & 3120 & 2780 & 2720 & 2080 & 2180 & 2660 \\
\hline
\end{tabular}




\begin{tabular}{l}
\hline Table 4.7 MHFS: Overall Cost In Fully Connected Topology \\
\hline \begin{tabular}{|c|ccccccccc|}
\hline Utilization & .3 & .4 & .5 & .6 & .7 & .8 & .9 & 1 \\
\cline { 2 - 8 } & Flows & & & & & & & & \\
1 & 480 & 480 & 480 & 480 & 480 & 480 & 480 & 480 \\
2 & 1320 & 1320 & 1320 & 1320 & 1320 & 1320 & 1320 & 1320 \\
3 & 1640 & 1220 & 1120 & 1220 & 1220 & 1320 & 1140 & 1120 \\
4 & 1660 & 1480 & 1420 & 1220 & 1120 & 1160 & 1080 & 1180 \\
5 & 2280 & 2040 & 1680 & 1760 & 1720 & 1480 & 1500 & 1280 \\
6 & 3200 & 2360 & 1980 & 2000 & 1680 & 2000 & 1640 & 1520 \\
7 & 3260 & 2520 & 1940 & 2060 & 1940 & 1800 & 1680 & 1380 \\
8 & 3700 & 2680 & 2400 & 2300 & 2120 & 2040 & 2320 & 1920 \\
9 & 4220 & 2900 & 2680 & 2480 & 2120 & 2000 & 2220 & 2060 \\
10 & 3820 & 3020 & 2400 & 2080 & 2000 & 1860 & 1880 & 1980 \\
11 & 4700 & 3440 & 3160 & 2860 & 2760 & 2560 & 2400 & 2340 \\
12 & 4360 & 3690 & 3020 & 2740 & 2880 & 2640 & 2800 & 2520 \\
\hline
\end{tabular}
\end{tabular}

Table 4.8 MHFS: Overall Cost In Random Topology
\begin{tabular}{|c|ccccccccc|}
\hline Utilization & .3 & .4 & .5 & .6 & .7 & .8 & .9 & 1 \\
\hline Flows & & & & & & & & & \\
1 & 420 & 420 & 420 & 420 & 420 & 420 & 420 & 420 \\
2 & 940 & 1000 & 1020 & 980 & 980 & 980 & 980 & 980 \\
3 & 1260 & 1300 & 1100 & 1000 & 1140 & 960 & 1140 & 900 \\
4 & 1580 & 1420 & 1200 & 1060 & 1060 & 1240 & 1260 & 1060 \\
5 & 1860 & 1780 & 1420 & 1280 & 1440 & 1160 & 1260 & 1000 \\
6 & 2160 & 2000 & 1800 & 1920 & 1520 & 1440 & 1360 & 1360 \\
7 & 2640 & 2180 & 1920 & 1700 & 1780 & 1700 & 1480 & 1800 \\
8 & 3120 & 2280 & 2000 & 1540 & 1360 & 1560 & 1620 & 1720 \\
9 & 3160 & 2620 & 2400 & 2020 & 2000 & 2000 & 1780 & 2120 \\
10 & 3200 & 2960 & 2480 & 2500 & 1940 & 2000 & 1800 & 1620 \\
11 & 3880 & 3260 & 2900 & 2360 & 2140 & 2280 & 2120 & 2040 \\
12 & 4340 & 3760 & 2620 & 2720 & 2540 & 2320 & 2420 & 1980 \\
\hline
\end{tabular}




\begin{tabular}{l}
\hline Table 4.9 MHFSTS: Overall Cost In Fully Connected Topology \\
\hline \begin{tabular}{|c|cccccccccc|}
\hline Utilization & .3 & .4 & .5 & .6 & .7 & .8 & .9 & 1 \\
\hline Flows & & & & & & & & \\
1 & 480 & 480 & 480 & 480 & 480 & 480 & 480 & 480 \\
2 & 1140 & 1100 & 980 & 1220 & 960 & 1140 & 1020 & 1020 \\
3 & 1700 & 1360 & 1320 & 1200 & 1320 & 1180 & 1220 & 1320 \\
4 & 1920 & 1680 & 1500 & 1260 & 1260 & 1440 & 1320 & 1320 \\
5 & 2620 & 1700 & 1700 & 1500 & 1500 & 1500 & 1500 & 1680 \\
6 & 3060 & 2380 & 2000 & 1660 & 1620 & 1540 & 1520 & 1560 \\
7 & 2920 & 2160 & 2220 & 1880 & 1900 & 1800 & 1820 & 1620 \\
8 & 3300 & 2760 & 2360 & 2500 & 2420 & 2400 & 2360 & 1840 \\
9 & 4000 & 3020 & 2680 & 2380 & 2260 & 2360 & 2100 & 2260 \\
10 & 3960 & 3140 & 3240 & 2800 & 2720 & 2500 & 2460 & 2420 \\
11 & 4660 & 3740 & 3000 & 2780 & 2960 & 2700 & 2700 & 2660 \\
12 & 4680 & 3660 & 3100 & 2880 & 2700 & 2520 & 2320 & 2440 \\
\hline
\end{tabular}
\end{tabular}

\begin{tabular}{c}
\hline Table 4.10 MHFSTS: Overall Cost In Random Topology \\
\hline \begin{tabular}{|c|cccccccccc|}
\hline Utilization & .3 & .4 & .5 & .6 & .7 & .8 & .9 & 1 \\
\hline Flows & & & & & & & & \\
1 & 420 & 420 & 420 & 420 & 420 & 420 & 420 & 420 \\
2 & 1060 & 980 & 960 & 1020 & 1020 & 1020 & 880 & 880 \\
3 & 1380 & 1200 & 1040 & 1060 & 1140 & 1000 & 1080 & 1000 \\
4 & 1320 & 1440 & 1380 & 1320 & 1160 & 880 & 1020 & 880 \\
5 & 1920 & 1580 & 1480 & 1620 & 1620 & 1080 & 1060 & 1220 \\
6 & 2420 & 2040 & 1840 & 1700 & 1560 & 1600 & 1360 & 1540 \\
7 & 2760 & 2160 & 1880 & 1580 & 1420 & 1440 & 1440 & 1620 \\
8 & 3000 & 2320 & 2200 & 1560 & 1580 & 1980 & 1680 & 1660 \\
9 & 3360 & 2720 & 2380 & 2100 & 2100 & 1940 & 1860 & 1960 \\
10 & 3580 & 2980 & 2460 & 2240 & 2100 & 2080 & 1920 & 1940 \\
11 & 4260 & 3260 & 2960 & 2960 & 2300 & 2060 & 2020 & 1880 \\
12 & 4340 & 3480 & 2800 & 2760 & 2240 & 2680 & 2640 & 2500 \\
\hline
\end{tabular}
\end{tabular}




\begin{tabular}{c}
\hline Table 4.11 Dijkstra-ILS: Overall Cost In Fully Connected Topology \\
\hline \begin{tabular}{|c|cccccccccc|}
\hline Utilization & .3 & .4 & .5 & .6 & .7 & .8 & .9 & 1 \\
\hline Flows & & & & & & & & & \\
1 & 540 & 540 & 540 & 540 & 540 & 540 & 540 & 540 \\
2 & 1560 & 1260 & 1260 & 960 & 960 & 960 & 960 & 960 \\
3 & 2720 & 2180 & 1680 & 1340 & 1340 & 1340 & 1080 & 1080 \\
4 & 2840 & 2340 & 1820 & 1500 & 1500 & 1260 & 1260 & 1260 \\
5 & 2460 & 2000 & 1480 & 1220 & 1220 & 1020 & 1020 & 1020 \\
6 & 2820 & 2240 & 1720 & 1380 & 1400 & 1140 & 1160 & 1180 \\
7 & 2900 & 2300 & 1720 & 1380 & 1380 & 1180 & 1180 & 1160 \\
8 & 3180 & 2520 & 1900 & 1620 & 1620 & 1440 & 1340 & 1360 \\
9 & 5580 & 4940 & 4100 & 3880 & 3880 & 3540 & 3540 & 3540 \\
10 & 5460 & 4700 & 4100 & 3600 & 3680 & 3360 & 3360 & 3360 \\
11 & 6980 & 5920 & 4820 & 4280 & 4280 & 4080 & 3980 & 3780 \\
12 & 7680 & 6060 & 5180 & 4320 & 4280 & 4120 & 4040 & 4040 \\
\hline
\end{tabular}
\end{tabular}

\begin{tabular}{c}
\hline Table 4.12 Dijkstra-ILS: Overall Cost In Random Topology \\
\hline \begin{tabular}{|c|ccccccccc|}
\hline Utilization & .3 & .4 & .5 & .6 & .7 & .8 & .9 & 1 \\
\hline Flows & & & & & & & & \\
1 & 420 & 420 & 420 & 420 & 420 & 420 & 420 & 420 \\
2 & 1320 & 1060 & 780 & 780 & 780 & 780 & 780 & 780 \\
3 & 2160 & 1780 & 1300 & 1100 & 1100 & 860 & 860 & 860 \\
4 & 2260 & 1840 & 1500 & 1160 & 1160 & 880 & 1020 & 880 \\
5 & 2600 & 2260 & 1840 & 1660 & 1640 & 1500 & 1500 & 1500 \\
6 & 3360 & 2900 & 2500 & 2220 & 2220 & 2020 & 2020 & 2000 \\
7 & 3440 & 2940 & 2520 & 2280 & 2280 & 2100 & 2100 & 2100 \\
8 & 3900 & 3260 & 2720 & 2520 & 2520 & 2320 & 2340 & 2320 \\
9 & 4380 & 3820 & 3280 & 2960 & 3000 & 2840 & 2820 & 2800 \\
10 & 4540 & 3880 & 3380 & 3220 & 3260 & 3080 & 3040 & 3040 \\
11 & 5420 & 4580 & 3920 & 3680 & 3640 & 3620 & 3480 & 3420 \\
12 & 6080 & 4800 & 4180 & 3560 & 3520 & 3380 & 3240 & 3340 \\
\hline
\end{tabular}
\end{tabular}




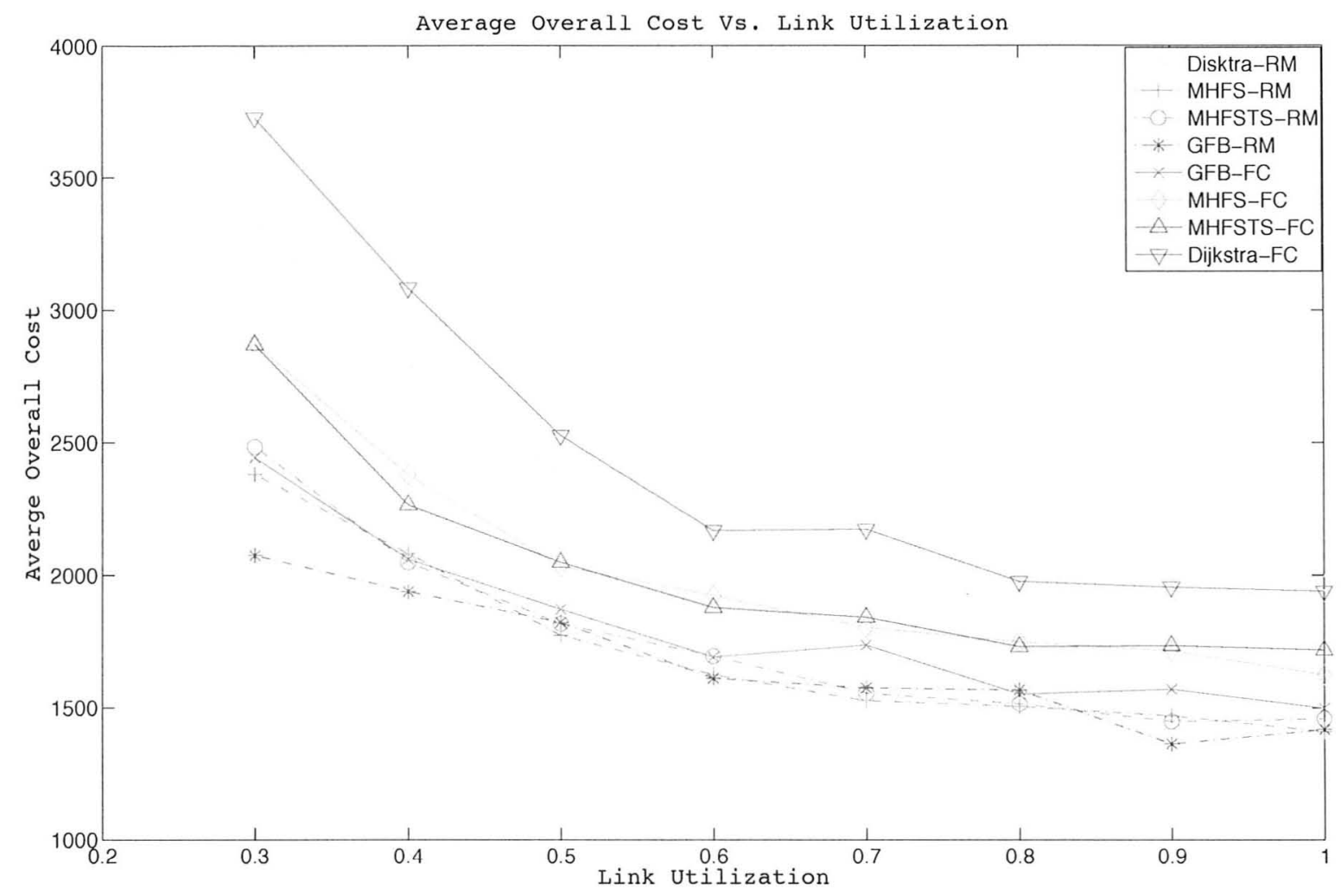

Figure 4.4: Overall Cost Of Deploying Secondary Network 


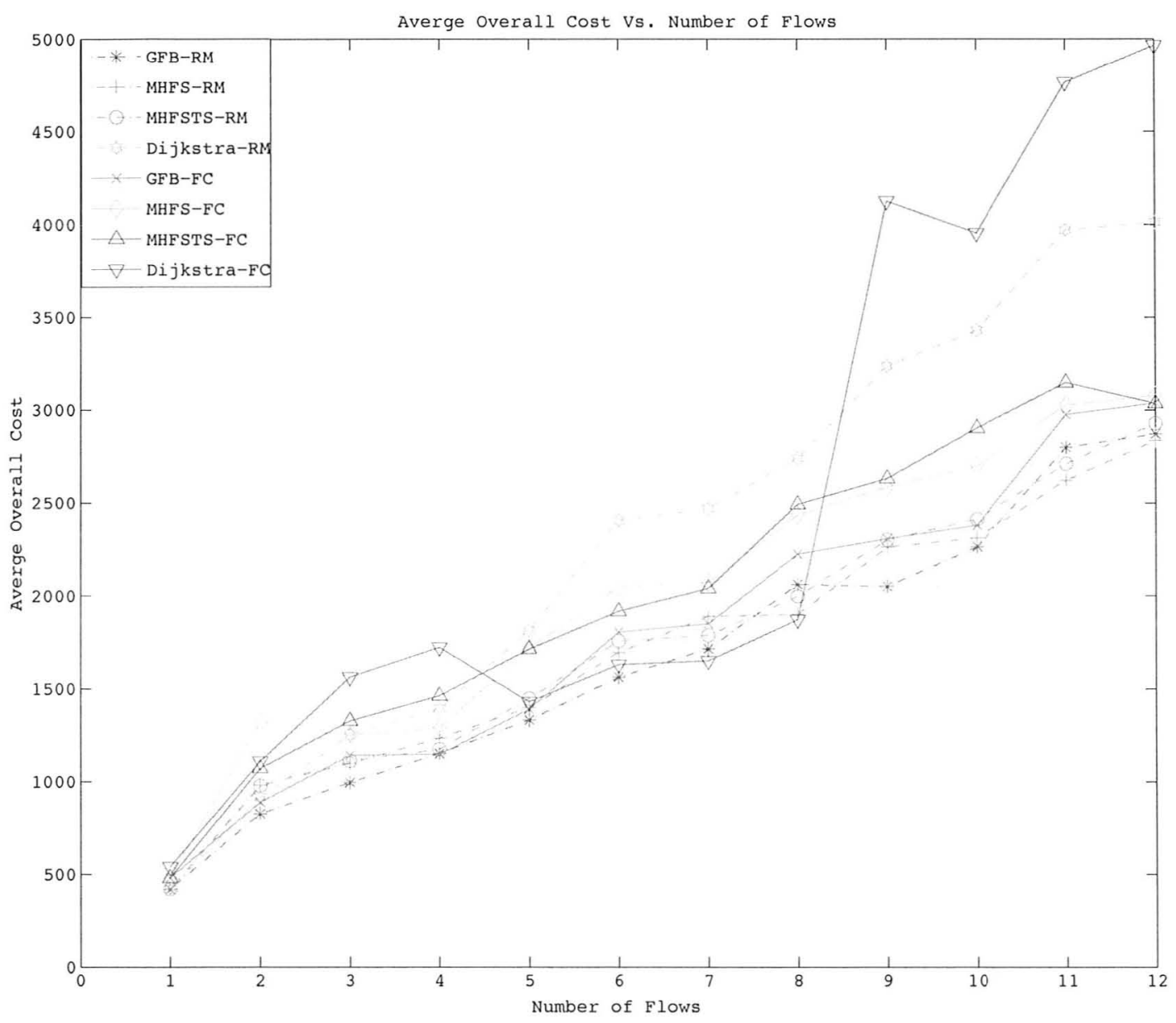

Figure 4.5: Overall Cost Of Deploying Secondary Network

In this subsection the tables and graphs show the overall cost of leasing frequencies resulted from solving the optimization problem for the two mentioned topologies. The first observation is that increasing link utilization decreases the overall cost. It can simply be explained considering the fact that higher link utilization allows multiple flows to be routed over an already bought link and thus a lower cost would be expected. The increase in the number of flows has also increased the costs. Comparing two topologies reveals the fact that the costs are higher for fully connected topologies. This is caused by the interference constraint in the optimization problem. Since 
it forces using a larger number of channels to avoid undesired interference over the primary network. All the heuristics show similar behavior under different topologies. The algorithms can be ranked in the increasing price order as below:

$1-\mathrm{GFB}$

2- MHFS

3- MHFSTS

4- Dijkstra-ILS

The GFB has the lowest average prices. It can be easily explained due to the fact that GFB's objective function is the overall cost, so it primarily tries to minimize the cost, while in the all other algorithms, first the shortest hop routing is performed, without considering the frequency prices, and then in local search step the costs are locally minimized. MHFS produces very close results to MHFSTS, since in both algorithms, the initial solution is generated by performing the constrained shortest path routing algorithm. The only difference is that in MHFS, in every step the routing of individual flows is found in all time slots, while in MHFSTS this process is performed in each time slot separately. Since there is no dependancy between traffic amount of a specific flow in consecutive time slots, the initial solutions to MHFSTS and MHFS are similar. Considering the same ILS is performed on the initial solutions in both algorithms, similar overall costs are to be expected.

Dijkstra-ILS stands last in the ranking. We should consider the fact that the initial results from Dijkstra's shortest path routing don't necessarily satisfy all the constraints. In the ILS step, the frequency assignment is conducted in a way that the final results be consistent with the constraints. Although the routes would be 
the shortest, ILS would have to assign more expensive frequencies to satisfy interference as well as other constraints. This might cause infeasibility problems in the ILS stage, specially when the primary network is highly congested. In order to avoid this problem, more exclusive channels should be used for the secondary network with higher prices. We increased the available channels for the secondary network to 14 for the fully connected topology and 10 for the random one to avoid infeasible solutions, when working with Dijkstra-ILS.

There are instances in the table that the overall pricing ranking doesn't hold. This is because all the designed heuristics don't consider all the flows at a time when finding the sub-optimum results. Thus for example, GFB might choose a path for Flow 2 that will cause Flow 3 to be routed over a very expensive route, while in MHFS a different route from GFB's might be chosen for Flow 2 that will cause Flow 3 to be routed over a much cheaper path. In this situation the overall cost in GFB would be higher, but the average values make a better judgement rather than individual results. 


\subsubsection{Number of Hops Tables}

\begin{tabular}{c}
\hline Table 4.13 GFB: Number Of Hops In Fully Connected Topology \\
\hline \\
$\qquad$\begin{tabular}{|c|ccccccccc|}
\hline Utilization & .3 & .4 & .5 & .6 & .7 & .8 & .9 & 1 \\
\hline Flows & & & & & & & & \\
1 & 15 & 15 & 15 & 15 & 15 & 15 & 15 & 15 \\
2 & 27 & 31 & 28 & 27 & 38 & 34 & 28 & 28 \\
3 & 39 & 39 & 43 & 43 & 50 & 40 & 47 & 49 \\
4 & 42 & 51 & 54 & 46 & 47 & 45 & 45 & 45 \\
5 & 55 & 74 & 64 & 63 & 63 & 63 & 67 & 63 \\
6 & 73 & 83 & 88 & 103 & 105 & 189 & 90 & 93 \\
7 & 83 & 95 & 105 & 95 & 95 & 89 & 100 & 91 \\
8 & 102 & 100 & 104 & 108 & 164 & 107 & 107 & 107 \\
9 & 99 & 109 & 118 & 117 & 142 & 117 & 134 & 130 \\
10 & 137 & 131 & 116 & 109 & 140 & 115 & 131 & 115 \\
11 & 158 & 136 & 139 & 136 & 154 & 148 & 208 & 163 \\
12 & 117 & 146 & 188 & 218 & 227 & 223 & 144 & 169 \\
\hline
\end{tabular}
\end{tabular}

\begin{tabular}{l}
\hline Table 4.14 GFB: Number Of Hops In Random Topology \\
\hline \\
$\qquad$\begin{tabular}{|c|ccccccccc|}
\hline Utilization & .3 & .4 & .5 & .6 & .7 & .8 & .9 & 1 \\
\hline Flows & & & & & & & & \\
1 & 15 & 15 & 15 & 15 & 15 & 15 & 15 & 15 \\
2 & 31 & 32 & 33 & 30 & 42 & 42 & 38 & 38 \\
3 & 40 & 47 & 48 & 52 & 58 & 61 & 45 & 69 \\
4 & 41 & 53 & 54 & 59 & 62 & 69 & 69 & 50 \\
5 & 49 & 65 & 61 & 71 & 83 & 90 & 63 & 65 \\
6 & 74 & 82 & 80 & 88 & 89 & 103 & 88 & 96 \\
7 & 77 & 80 & 89 & 82 & 108 & 109 & 82 & 84 \\
8 & 100 & 96 & 106 & 100 & 105 & 134 & 99 & 122 \\
9 & 67 & 104 & 114 & 117 & 114 & 126 & 134 & 114 \\
10 & 119 & 119 & 122 & 125 & 147 & 158 & 146 & 143 \\
11 & 126 & 129 & 143 & 163 & 154 & 164 & 161 & 170 \\
12 & 130 & 154 & 167 & 171 & 172 & 162 & 154 & 219 \\
\hline
\end{tabular}
\end{tabular}




\begin{tabular}{c}
\hline \multicolumn{10}{c}{ Table 4.15 MHFS: Number Of Hops In Fully Connected Topology } \\
\hline \begin{tabular}{|c|ccccccccc|}
\hline Utilization & .3 & .4 & .5 & .6 & .7 & .8 & .9 & 1 \\
\cline { 2 - 10 } & Flows & & & & & & & & \\
1 & 15 & 15 & 15 & 16 & 16 & 16 & 16 & 16 \\
2 & 27 & 27 & 32 & 31 & 30 & 29 & 36 & 36 \\
3 & 37 & 39 & 41 & 44 & 41 & 42 & 45 & 43 \\
4 & 42 & 48 & 43 & 53 & 48 & 49 & 49 & 48 \\
5 & 51 & 51 & 54 & 57 & 55 & 61 & 57 & 70 \\
6 & 72 & 72 & 72 & 74 & 72 & 72 & 72 & 82 \\
7 & 69 & 73 & 75 & 78 & 75 & 70 & 81 & 81 \\
8 & 77 & 84 & 83 & 86 & 88 & 87 & 89 & 93 \\
9 & 97 & 89 & 92 & 105 & 97 & 94 & 87 & 95 \\
10 & 91 & 95 & 95 & 89 & 95 & 95 & 96 & 95 \\
11 & 111 & 108 & 114 & 103 & 115 & 109 & 137 & 110 \\
12 & 131 & 131 & 131 & 117 & 128 & 134 & 139 & 136 \\
\hline
\end{tabular}
\end{tabular}

Table 4.16 MHFS: Number Of Hops In Random Topology

\begin{tabular}{|c|ccccccccc|}
\hline Utilization & .3 & .4 & .5 & .6 & .7 & .8 & .9 & 1 \\
\hline Flows & & & & & & & & \\
1 & 15 & 15 & 15 & 15 & 15 & 15 & 15 & 15 \\
2 & 28 & 31 & 27 & 27 & 27 & 27 & 27 & 27 \\
3 & 37 & 42 & 38 & NR & 53 & 45 & 45 & 41 \\
4 & 40 & 51 & 46 & 49 & 46 & 45 & 51 & 48 \\
5 & 52 & 47 & 58 & 52 & 52 & 65 & 52 & 59 \\
6 & 63 & 69 & 67 & 89 & 74 & 74 & 72 & 72 \\
7 & 67 & 79 & 73 & 77 & 73 & 84 & 82 & 73 \\
8 & 77 & 85 & 86 & 89 & 91 & 80 & 83 & 82 \\
9 & 87 & 92 & 95 & 90 & 95 & 95 & 94 & 112 \\
10 & 83 & 96 & 98 & 92 & 110 & 95 & 106 & 131 \\
11 & 100 & 112 & 101 & 147 & 128 & 120 & 119 & 118 \\
12 & 110 & 119 & 121 & 118 & 125 & 147 & 148 & 141 \\
\hline
\end{tabular}




\begin{tabular}{c}
\hline Table 4.17 MHFSTS: Number Of Hops In Fully Connected Topology \\
\hline \begin{tabular}{|c|ccccccccc|}
\hline Utilization & .3 & .4 & .5 & .6 & .7 & .8 & .9 & 1 \\
\cline { 2 - 10 } & Flows & & & & & & & & \\
1 & 15 & 15 & 15 & 15 & 15 & 15 & 15 & 15 \\
2 & 27 & 27 & 32 & 31 & 30 & 29 & 36 & 36 \\
3 & 38 & 39 & 39 & 45 & 42 & 42 & 41 & 42 \\
4 & 41 & 48 & 43 & 53 & 48 & 49 & 49 & 48 \\
5 & 49 & 53 & 50 & 51 & 51 & 53 & 51 & 57 \\
6 & 66 & 79 & 75 & 81 & 75 & 80 & 79 & 75 \\
7 & 69 & 79 & 75 & 81 & 75 & 80 & 79 & 75 \\
8 & 80 & 81 & 86 & 85 & 84 & 85 & 84 & 89 \\
9 & 105 & 91 & 88 & 90 & 98 & 91 & 92 & 95 \\
10 & 92 & 90 & 95 & 89 & 95 & 95 & 96 & 95 \\
11 & 111 & 112 & 107 & 99 & 126 & 104 & 104 & 103 \\
12 & 112 & 116 & 129 & 122 & 128 & 135 & 139 & 141 \\
\hline
\end{tabular}
\end{tabular}

\section{Table 4.18 MHFSTS: Number Of Hops In Random Topology}

\begin{tabular}{|c|ccccccccc|}
\hline Utilization & .3 & .4 & .5 & .6 & .7 & .8 & .9 & 1 \\
\hline Flows & & & & & & & & \\
1 & 15 & 15 & 15 & 15 & 15 & 15 & 15 & 15 \\
2 & 28 & 31 & 31 & 29 & 29 & 27 & 29 & 29 \\
3 & 39 & 40 & 41 & 44 & 53 & 45 & 39 & 43 \\
4 & 40 & 44 & 44 & 43 & 49 & 49 & 45 & 51 \\
5 & 50 & 54 & 52 & 49 & 49 & 56 & 60 & 60 \\
6 & 67 & 68 & 68 & 73 & 71 & 75 & 79 & 78 \\
7 & 75 & 72 & 74 & 77 & 75 & 75 & 90 & 78 \\
8 & 79 & 85 & 84 & 85 & 95 & 91 & 89 & 97 \\
9 & 86 & 90 & 87 & 98 & 104 & 108 & 111 & 107 \\
10 & 88 & 92 & 88 & 103 & 103 & 117 & 104 & 107 \\
11 & 103 & 109 & 110 & 110 & 123 & 126 & 132 & 126 \\
12 & 110 & 110 & 119 & 121 & 113 & 124 & 129 & 131 \\
\hline
\end{tabular}




\begin{tabular}{l}
\hline Table 4.19 Dijkstra-ILS: Number Of Hops In Fully Connected Topology \\
\hline \begin{tabular}{|c|ccccccccc|}
\hline Utilization & .3 & .4 & .5 & .6 & .7 & .8 & .9 & 1 \\
\hline Flows & & & & & & & & \\
1 & 15 & 15 & 15 & 15 & 15 & 15 & 15 & 15 \\
2 & 33 & 33 & 33 & 33 & 33 & 33 & 33 & 33 \\
3 & 45 & 45 & 45 & 45 & 45 & 45 & 45 & 45 \\
4 & 48 & 48 & 48 & 50 & 52 & 48 & 48 & 48 \\
5 & 54 & 54 & 54 & 56 & 56 & 54 & 54 & 54 \\
6 & 69 & 69 & 75 & 75 & 75 & 69 & 69 & 69 \\
7 & 74 & 72 & 72 & 74 & 72 & 72 & 72 & 72 \\
8 & 81 & 81 & 81 & 81 & 81 & 81 & 81 & 81 \\
9 & 87 & 87 & 89 & 87 & 87 & 87 & 87 & 87 \\
10 & 90 & 96 & 92 & 94 & 90 & 90 & 90 & 90 \\
11 & 105 & 111 & 107 & 105 & 107 & 105 & 105 & 105 \\
12 & 130 & 126 & 128 & 126 & 128 & 126 & 128 & 126 \\
\hline
\end{tabular}
\end{tabular}

Table 4.20 Dijkstra-ILS: Number Of Hops In Random Topology

\begin{tabular}{|c|cccccccc|}
\hline Utilization & .3 & .4 & .5 & .6 & .7 & .8 & .9 & 1 \\
\hline Flows & & & & & & & & \\
1 & 15 & 15 & 15 & 15 & 15 & 15 & 15 & 15 \\
2 & 33 & 33 & 33 & 33 & 33 & 33 & 33 & 33 \\
3 & 45 & 45 & 45 & 45 & 45 & 45 & 45 & 45 \\
4 & 50 & 50 & 48 & 50 & 48 & 48 & 48 & 48 \\
5 & 54 & 56 & 56 & 56 & 54 & 54 & 54 & 54 \\
6 & 69 & 69 & 73 & 69 & 69 & 69 & 69 & 69 \\
7 & 74 & 72 & 72 & 72 & 74 & 72 & 72 & 72 \\
8 & 81 & 83 & 83 & 83 & 81 & 81 & 81 & 81 \\
9 & 89 & 87 & 91 & 87 & 87 & 87 & 87 & 87 \\
10 & 94 & 96 & 98 & 90 & 90 & 90 & 90 & 90 \\
11 & 105 & 107 & 107 & 105 & 105 & 105 & 105 & 105 \\
12 & 128 & 136 & 130 & 128 & 134 & 130 & 126 & 134 \\
\hline
\end{tabular}




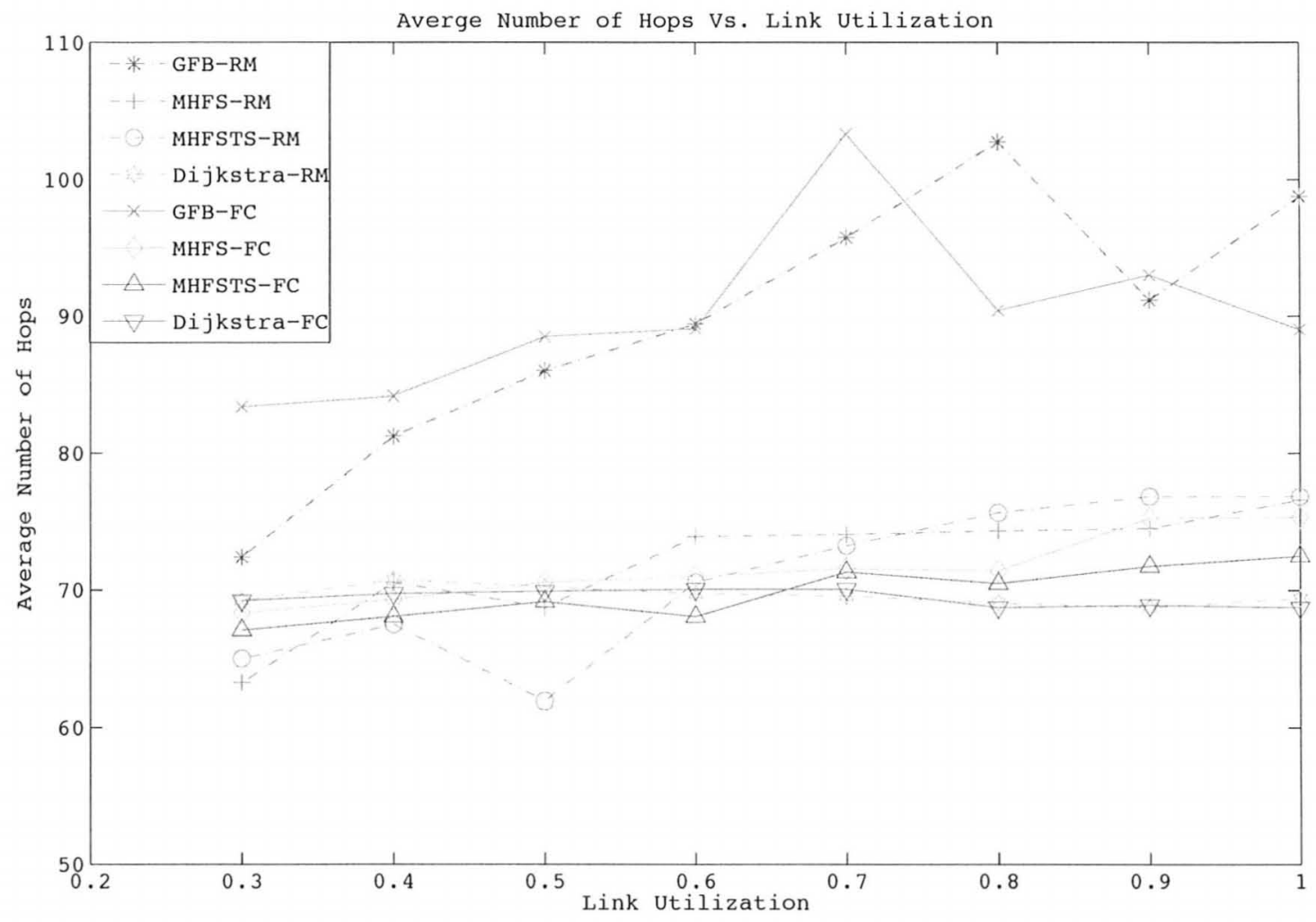

Figure 4.6: Total Number Of Hops For Routing Secondary Network Traffic 


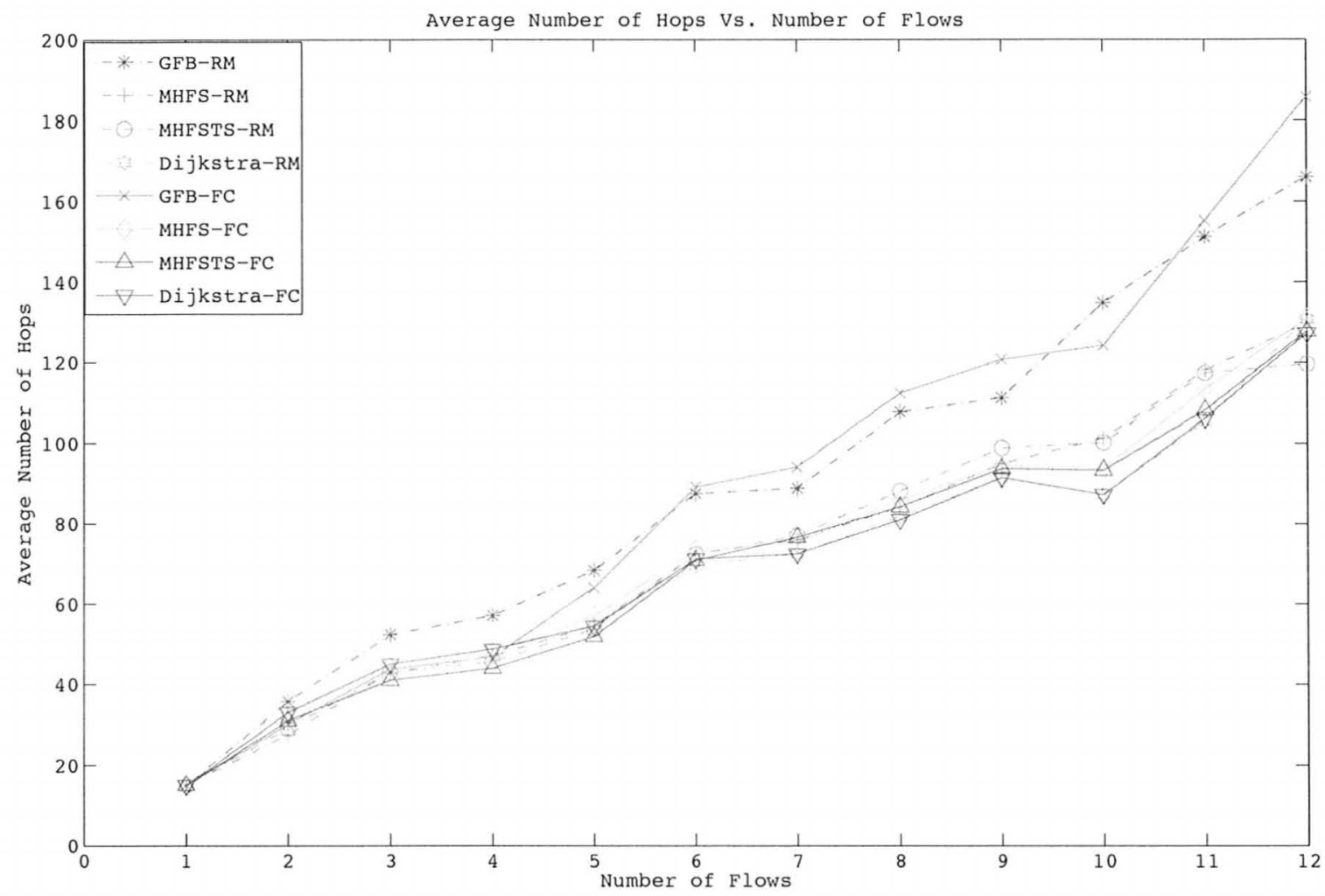

Figure 4.7: Total Number Of Hops For Routing Secondary Network Traffic

In this subsection the number of hops used in the routings are compared for the four heuristics. Increasing the number of flows has increased the number of hops. Increasing link utilization has had different effects over different algorithms. For GFB, reduction in the number of hops is observed, while for MHFS, MHFSTS and DijkstraILS, it has not changed significantly. The ranking of algorithms considering hop count can be written as below:

1- Dijkstra-ILS

2- MHFS

3- MHFSTS

$4-$ GFB 
GFB generates the longest routes, which can be easily justified according to the fact that the objective only considers the frequency prices, while in other heuristics the initial solutions are generated using shortest path algorithms and objective functions. This would make the final routings inherently shorter than the ones generated by GFB. Dijkstra-ILS has the shortest paths. This is because the initial solution of the Dijkstra algorithm just considers finding the shortest paths without being limited to the constraints, while MHFS and MHFSTS find constrained shortest path routes as initial solutions. Comparing MHFS and MHFSTS would show that they have very close numbers of hops, which is because of the very similar initial solutions that they have.

An interesting point that can be observed is the variance of the number of hops. As can be seen, other than GFB, the number of hops doesn't change drastically with increasing the link utility. This is because of both the initial solutions and the ILS step. We should consider that ILS only improves the results locally, and thus can't change the number of hops greatly, while in GFB, since there is no initial solution and the objective concentrates on reducing the costs, the optimizer prefers a longer path with a lower cost than a shorter one with a higher price. This is opposite to the way that the initial solutions are generated in the other algorithms.

An interesting point to note is how close results from Dijkstra-ILS, MHFS and MHFSTS are for the two topologies. This is basically because of the initial solutions that each of these algorithms have. The initial solutions all focus on finding the shortest paths, so the number of hops would be very close in the initial solutions, regardless of link utilization. Since even if less expensive links are occupied or suffer from high 
interference, the initial solution tends to route the traffic over more expensive, but at the same time shortest, hops. Thus the number of hops would be very close for both topologies and various link utilizations.

\subsubsection{Number of Deployed Nodes Tables}

\begin{tabular}{l}
\hline Table 4.21 GFB: Number Of Deployed Nodes In Fully Connected Topology \\
\hline \\
$\qquad$\begin{tabular}{c|ccccccccc}
\hline Utilization & .3 & .4 & .5 & .6 & .7 & .8 & .9 & 1 \\
\hline Flows & & & & & & & & \\
1 & 6 & 6 & 6 & 6 & 6 & 6 & 6 & 6 \\
2 & 11 & 11 & 11 & 11 & 11 & 11 & 13 & 13 \\
3 & 15 & 15 & 12 & 12 & 12 & 12 & 12 & 12 \\
4 & 12 & 15 & 12 & 12 & 12 & 12 & 12 & 12 \\
5 & 15 & 16 & 15 & 14 & 14 & 14 & 14 & 14 \\
6 & 21 & 19 & 19 & 19 & 18 & 17 & 15 & 17 \\
7 & 21 & 20 & 20 & 19 & 19 & 18 & 19 & 14 \\
8 & 24 & 23 & 20 & 19 & 19 & 19 & 20 & 20 \\
9 & 23 & 23 & 21 & 20 & 21 & 20 & 21 & 21 \\
10 & 13 & 23 & 21 & 21 & 22 & 20 & 20 & 19 \\
11 & 24 & 24 & 22 & 21 & 21 & 22 & 21 & 20 \\
12 & 23 & 24 & 25 & 22 & 21 & 21 & 19 & 21 \\
\hline
\end{tabular}
\end{tabular}

Table 4.22 GFB: Number Of Deployed Nodes In Random Topology

\begin{tabular}{|c|cccccccc|}
\hline Utilization & .3 & .4 & .5 & .6 & .7 & .8 & .9 & 1 \\
\hline Flows & & & & & & & & \\
1 & 6 & 6 & 6 & 6 & 6 & 6 & 6 & 6 \\
2 & 13 & 12 & 12 & 8 & 10 & 11 & 9 & 9 \\
3 & 14 & $?$ & 13 & 12 & 12 & 12 & 9 & 11 \\
4 & 14 & 15 & 13 & 12 & 14 & 11 & 11 & 10 \\
5 & 15 & 15 & 13 & 14 & 15 & 14 & 10 & 12 \\
6 & 19 & 17 & 19 & 15 & 17 & 16 & 12 & 12 \\
7 & 20 & 21 & 19 & 18 & 19 & 17 & 16 & 16 \\
8 & 21 & 21 & 23 & 20 & 19 & 20 & 18 & 17 \\
9 & 19 & 21 & 23 & 20 & 19 & 20 & 18 & 17 \\
10 & 23 & 23 & 21 & 21 & 19 & 21 & 17 & 17 \\
11 & 22 & 23 & 22 & 23 & 20 & 18 & 21 & 19 \\
12 & 23 & 22 & 22 & 21 & 21 & 21 & 21 & 20 \\
\hline
\end{tabular}


Table 4.23 MHFS: Number Of Deployed Nodes In Fully Connected Topology

\begin{tabular}{|c|cccccccc|}
\hline Utilization & .3 & .4 & .5 & .6 & .7 & .8 & .9 & 1 \\
\hline Flows & & & & & & & & \\
1 & 6 & 6 & 6 & 6 & 6 & 6 & 6 & 6 \\
2 & 12 & 13 & 12 & 12 & 13 & 12 & 10 & 10 \\
3 & 11 & 11 & 12 & 13 & 12 & 13 & 11 & 13 \\
4 & 13 & 13 & 13 & 13 & 12 & 12 & 11 & 12 \\
5 & 15 & 13 & 12 & 13 & 13 & 13 & 12 & 12 \\
6 & 17 & 15 & 15 & 13 & 15 & 14 & 14 & 14 \\
7 & 16 & 16 & 16 & 16 & 16 & 17 & 16 & 16 \\
8 & 16 & 16 & 17 & 16 & 15 & 16 & 16 & 17 \\
9 & 20 & 17 & 19 & 19 & 17 & 17 & 18 & 17 \\
10 & 21 & 19 & 20 & 17 & 17 & 19 & 16 & 18 \\
11 & 22 & 22 & 19 & 19 & 19 & 19 & 19 & 19 \\
12 & 21 & 20 & 19 & 20 & 20 & 21 & 21 & 20 \\
\hline
\end{tabular}

Table 4.24 MHFS: Number Of Deployed Nodes In Random Topology

\begin{tabular}{|c|cccccccc|}
\hline Utilization & .3 & .4 & .5 & .6 & .7 & .8 & .9 & 1 \\
\hline Flows & & & & & & & & \\
1 & 6 & 6 & 6 & 6 & 6 & 6 & 6 & 6 \\
2 & 10 & 10 & 10 & 10 & 10 & 10 & 10 & 10 \\
3 & 12 & 13 & 11 & 11 & 12 & 10 & 12 & 10 \\
4 & 12 & 13 & 11 & 11 & 11 & 12 & 14 & 11 \\
5 & 14 & 13 & 12 & 13 & 13 & 12 & 12 & 10 \\
6 & 18 & 15 & 14 & 19 & 13 & 13 & 14 & 14 \\
7 & 18 & 16 & 17 & 17 & 19 & 16 & 17 & 15 \\
8 & 19 & 17 & 16 & 16 & 16 & 17 & 17 & 17 \\
9 & 17 & 17 & 19 & 18 & 18 & 17 & 17 & 17 \\
10 & 20 & 17 & 16 & 16 & 17 & 17 & 17 & 17 \\
11 & 22 & 20 & 17 & 22 & 18 & 18 & 18 & 17 \\
12 & 20 & 21 & 20 & 20 & 20 & 19 & 20 & 18 \\
\hline
\end{tabular}




\begin{tabular}{c}
\hline Table 4.25 MHFSTS: Number Of Deployed Nodes In Fully Connected Topology \\
\hline \begin{tabular}{|c|ccccccccc|}
\hline Utilization & .3 & .4 & .5 & .6 & .7 & .8 & .9 & 1 \\
\hline Flows & & & & & & & & \\
1 & 6 & 6 & 6 & 6 & 6 & 6 & 6 & 6 \\
2 & 10 & 9 & 11 & 12 & 10 & 11 & 11 & 11 \\
3 & 12 & 12 & 11 & 12 & 13 & 12 & 12 & 12 \\
4 & 12 & 12 & 12 & 12 & 12 & 12 & 12 & 11 \\
5 & 14 & 14 & 14 & 13 & 13 & 11 & 12 & 12 \\
6 & 16 & 15 & 16 & 15 & 15 & 16 & 14 & 15 \\
7 & 18 & 17 & 15 & 16 & 16 & 16 & 16 & 16 \\
8 & 16 & 16 & 17 & 16 & 15 & 16 & 16 & 17 \\
9 & 19 & 20 & 19 & 18 & 18 & 18 & 18 & 19 \\
10 & 19 & 18 & 18 & 18 & 18 & 18 & 18 & 18 \\
11 & 20 & 20 & 19 & 20 & 20 & 20 & 20 & 20 \\
12 & 19 & 19 & 20 & 21 & 19 & 20 & 22 & 21 \\
\hline
\end{tabular}
\end{tabular}

Table 4.26 MHFSTS: Number Of Deployed Nodes In Random Topology

\begin{tabular}{|c|cccccccc|}
\hline Utilization & .3 & .4 & .5 & .6 & .7 & .8 & .9 & 1 \\
\hline Flows & & & & & & & & \\
1 & 6 & 6 & 6 & 6 & 6 & 6 & 6 & 6 \\
2 & 9 & 10 & 9 & 11 & 11 & 9 & 10 & 10 \\
3 & 13 & 13 & 11 & 13 & 12 & 12 & 11 & 11 \\
4 & 13 & 15 & 12 & 12 & 12 & 10 & 10 & 10 \\
5 & 14 & 14 & 14 & 13 & 13 & 11 & 12 & 12 \\
6 & 16 & 15 & 16 & 15 & 15 & 16 & 14 & 15 \\
7 & 16 & 15 & 16 & 16 & 15 & 15 & 16 & 17 \\
8 & 17 & 18 & 17 & 17 & 17 & 19 & 16 & 17 \\
9 & 19 & 17 & 16 & 18 & 19 & 19 & 18 & 19 \\
10 & 18 & 18 & 18 & 18 & 18 & 17 & 18 & 18 \\
11 & NR & 20 & 17 & 17 & 19 & 18 & 18 & 18 \\
12 & 20 & 20 & 20 & 20 & 19 & 20 & 20 & 20 \\
\hline
\end{tabular}


Table 4.27 Dijkstra-ILS: Number Of Deployed Nodes In Fully Connected Topology

\begin{tabular}{|c|cccccccc|}
\hline Utilization & .3 & .4 & .5 & .6 & .7 & .8 & .9 & 1 \\
\hline Flows & & & & & & & & \\
1 & 6 & 6 & 6 & 6 & 6 & 6 & 6 & 6 \\
2 & 9 & 9 & 9 & 9 & 9 & 9 & 9 & 9 \\
3 & 10 & 10 & 10 & 10 & 10 & 10 & 10 & 10 \\
4 & 11 & 11 & 11 & 11 & 11 & 11 & 11 & 11 \\
5 & 12 & 12 & 12 & 12 & 12 & 12 & 12 & 12 \\
6 & 16 & 16 & 16 & 16 & 16 & 16 & 16 & 16 \\
7 & 18 & 18 & 18 & 18 & 18 & 18 & 18 & 18 \\
8 & 19 & 19 & 19 & 19 & 19 & 19 & 19 & 19 \\
9 & 20 & 20 & 20 & 20 & 20 & 20 & 20 & 20 \\
10 & 20 & 20 & 20 & 20 & 20 & 20 & 20 & 20 \\
11 & 21 & 21 & 21 & 21 & 21 & 21 & 21 & 21 \\
12 & 21 & 21 & 21 & 21 & 21 & 21 & 21 & 21 \\
\hline
\end{tabular}

Table 4.28 Dijkstra-ILS: Number Of Deployed Nodes In Random Topology

\begin{tabular}{|c|c|ccccccccc|}
\hline Utilization & .3 & .4 & .5 & .6 & .7 & .8 & .9 & 1 \\
\hline Flows & & & & & & & & \\
1 & 6 & 6 & 6 & 6 & 6 & 6 & 6 & 6 \\
2 & 9 & 9 & 9 & 9 & 9 & 9 & 9 & 9 \\
3 & 10 & 10 & 10 & 10 & 10 & 10 & 10 & 10 \\
4 & 11 & 11 & 11 & 11 & 11 & 11 & 11 & 11 \\
5 & 12 & 12 & 12 & 12 & 12 & 12 & 12 & 12 \\
6 & 16 & 16 & 16 & 16 & 16 & 16 & 16 & 16 \\
7 & 18 & 18 & 18 & 18 & 18 & 18 & 18 & 18 \\
8 & 19 & 19 & 19 & 19 & 19 & 19 & 19 & 19 \\
9 & 20 & 20 & 20 & 20 & 20 & 20 & 20 & 20 \\
10 & 20 & 20 & 20 & 20 & 20 & 20 & 20 & 20 \\
11 & 21 & 21 & 21 & 21 & 21 & 21 & 21 & 21 \\
12 & 21 & 21 & 21 & 21 & 21 & 21 & 21 & 21 \\
\hline
\end{tabular}




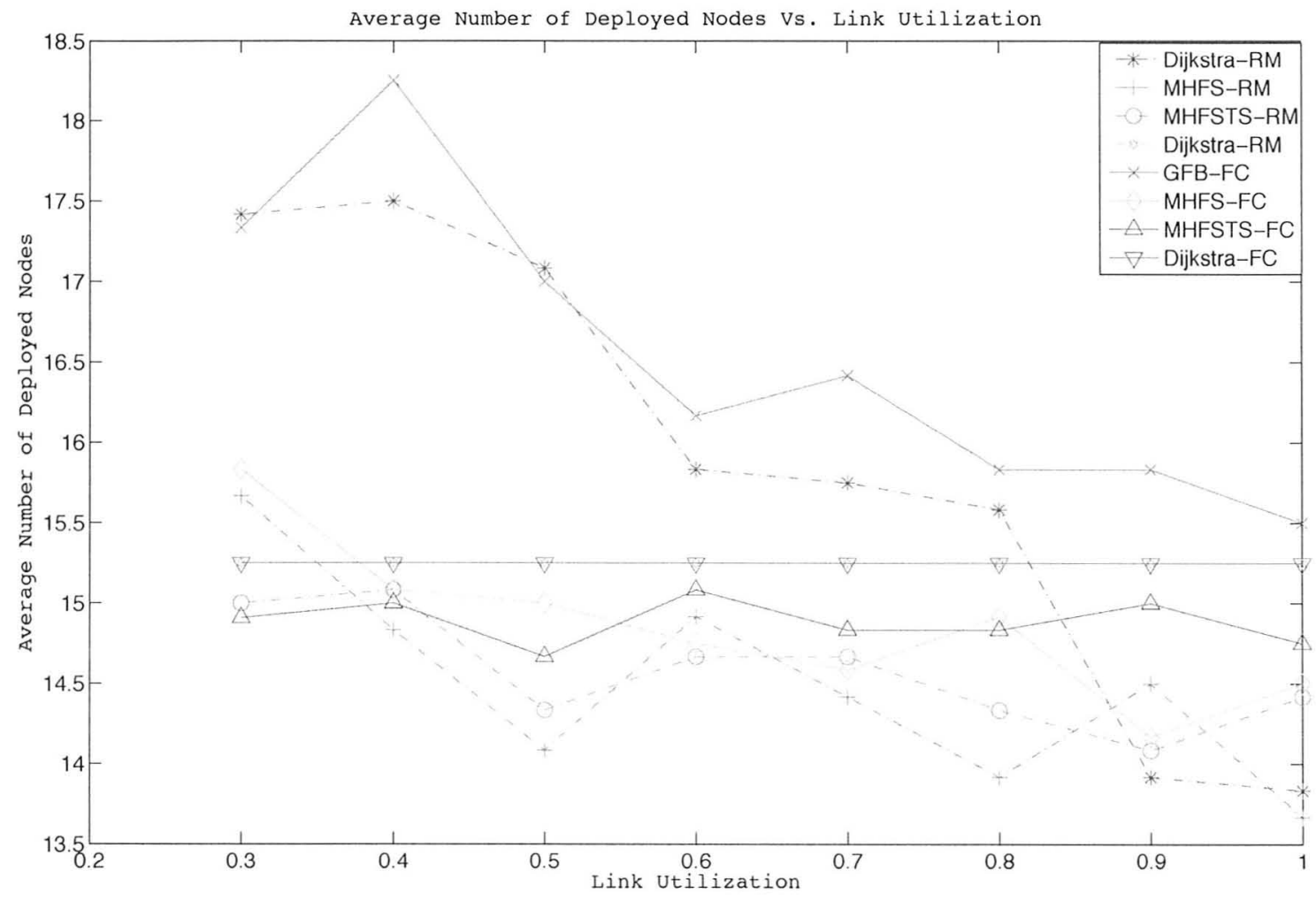

Figure 4.8: Total Number Of Deployed Secondary Nodes 


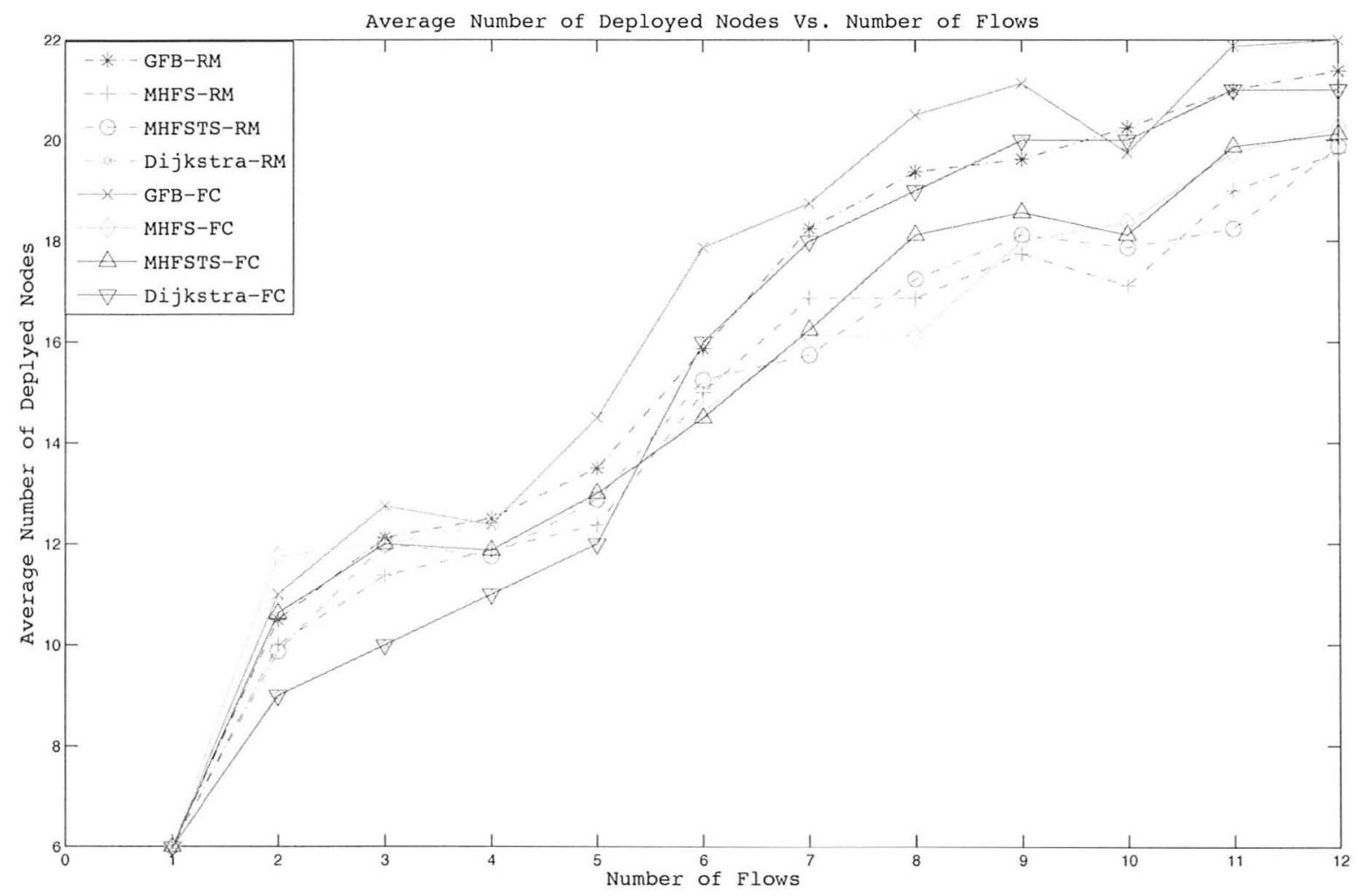

Figure 4.9: Total Number Of Deployed Secondary Nodes

The tables presented here include the number of secondary nodes required to be deployed for implementing the solutions generated by the four heuristics. Very similar to the number of hops comparison, the number of deployed nodes decreases with a drop in the number of flows. Except GFB, for other algorithms the number of deployed nodes has not changed drastically with increase in link utility. The ranking of algorithms considering the number of deployed nodes can be written as below:

$1-$ MHFS

2- MHFSTS

3-Dijkstra-ILS

4- GFB 
As in the previous subsection, GFB again falls behind the other three heuristics due to its priority in finding the least expensive routing and frequency allocations. As mentioned in the previous subsection, GFB generates longest paths which intuitively would cause higher numbers of nodes to be deployed.

It was mentioned in the previous subsection that Dijkstra-ILS produces shortest paths, so it should result in a lower number of required nodes. This would be correct if the objective functions of MHFS and MHFSTS were only the summation of hops, which is not the case. We have set the objective of MHFS and MHFSTS in the initial solution finding step to be both the summation of the number of hops as well as the total number of deployed nodes. Thus the initial solutions of MHFS and MHFSTS would not only be the constrained shortest paths, but also require the least number of deployed nodes. That's why we see that Dijkstra-ILS can't beat the other two algorithms in finding the least number of secondary nodes.

Very similar to the hop numbers comparison, Dijkstra-ILS, MHFS and MHFSTS for the two topologies reveals close numbers, which is because of the very similar initial solutions that they use. 


\section{Chapter 5}

\section{Conclusions and Future Work}

In this thesis we have considered the design of secondary wireless mesh networks where frequency channels are obtained by leasing from a primary bandwidth owner. The usage of each channel is subject to interference constraints so that the primary user is not unduly affected by the operation of the secondary user. When the network is designed and deployed, the secondary user would like to minimize the costs of using the required resources while satisfying its own traffic and interference requirements. This problem was formulated as a mixed integer optimization that describes the secondary node positioning, routing, and frequency allocations, subject to various constraints.

Due to the problem's high complexity, the optimum design can only be found for small problem sizes. To obtain more practical deployments, four heuristic algorithms were proposed and their performance was compared to solutions obtained from the optimization. The first algorithm is a greedy flow-based scheme (GFB) which iterates over the individual flows in each time slot based on solving a much simpler optimization at each step. The second algorithm (MHFS) uses an iterated location 
search whose initial solution is based on constrained shortest hop routing performed on the individual flows. MHFSTS was a simplified version of MHFS, in which the initial solution was found by performing shortest hop routing in individual time slots. A faster and more efficient algorithm was introduced based on Dijkstra's shortest path algorithm and Iterated Local Search which reduced the computation size of the problem to quadratic order.

Our results showed that the algorithms perform well for a variety of network scenarios. It was also shown using different examples that when considering the overall cost, GFB outperforms the other algorithms, while MHFS, MHFSTS and DijkstraILS generate more costly results, respectively. The reduction in computation size using the proposed algorithms was also discussed, and it was found that DijkstraILS is the fastest algorithm with the computation complexity of quadratic order, while the remaining algorithms are NP hard. Examples also showed that reducing link utilization, increasing the number of traffic flows and expanding the secondary network size increases the overall network price on average.

This research can be extended in various ways.

- Time varying primary network: In this research, we assumed that the primary network's topology would remain the same during the secondary network operation. This assumption was made to simplify the computation size of the problem. In real life scenarios, the primary network's topology might change according to its traffic pattern. This effect can be explored by using a time varying model of the primary network.

- Cooperative/Noncooperative secondary networks: Since the unlicensed operation over TV bands is desirable, there is the possibility that multiple secondary 
networks try to reach the channels simultaneously. Complications would arise in this scenario because these networks may act selfishly. The idea of cooperative or non-cooperative spectrum sharing can be applied here. Coordination is required both between competing secondaries as well as between secondaries and the primary.

- Adaptation to traffic changes: Since in the real life scenarios traffic flows arrive nondeterministically, the secondary network should be able to adapt to the changes that occur. One idea is to use learning algorithms, so that the secondary network can predict future changes and modify its configuration to better accommodate them. Because the traffics usually follow a certain distribution, and knowing this distribution would help better utilize the shared channels.

- Designing heuristics based on Genetic Algorithms: Heuristics based on genetic algorithms can be investigated to be used in this research. The problem with genetic algorithm in this research is that we are doing joint node placement, frequency assignment and routing at the same time. Unless an algorithm is designed to guide the Genetic Algorithm to the suboptimal solution, the GA has to explore all the options, which results in exponential computation complexity. One way to do this is to separate the routing and frequency assignment phases and perform GA over them. Again this will not solve all the problems, since the optimization problem is constrained and the interference constraint, which for every link depends on the existence of all the other links at the same frequency, will be problematic. 


\section{Bibliography}

[1] "Report of the Spectrum Efficiency Working Group". Technical report, FCC, November 2002.

[2] FCC. "Unlicensed Operation in the TV Broadcast Bands, Second Order and Report". ET Docket No.04-186, 2008.

[3] IEEE P802.22 Wireless RANs. "DTV Signal Sensing Using Pilot Detection". IEEE 802.22 Work Group, 2007.

[4] X. Li and S.A. Zekavat. "Traffic Pattern Prediction and Performance Investigation for Cognitive Radio Systems". IEEE Wireless Communications and Networking Conference, 2008. WCNC 2008., pages 894-899, April 2008.

[5] J.Sun and Q.H. Wu. "A Non-Cooperative Power Control Game Via New Pricing in Cognitive Radio". In International Conference on Wireless Communications Signal Processing, 2009. WCSP 2009., pages 1 -5, November 2009.

[6] D. Niyato and E. Hossain. "Market-Equilibrium, Competitive, and Cooperative Pricing for Spectrum Sharing in Cognitive Radio Networks: Analysis and Comparison". IEEE Transactions on Wireless Communications, , 7(11):4273 -4283, November 2008. 
[7] N.Li, L.Gong, and S.Li. "Price-Based Spectrum-Allocation Relay Routing in Cognitive Radio Networks". In 7th International Conference on Information, Communications and Signal Processing, 2009. ICICS 2009., pages 1 -5, December 2009 .

[8] D. Niyato and E. Hossain. "Competitive Pricing for Spectrum Sharing in Cognitive Radio Networks: Dynamic Game, Inefficiency of Nash Equilibrium, and Collusion". IEEE Journal on Selected Areas in Communications,, 26(1):192 202, Jan. 2008.

[9] W.Wang, T.Peng, and W.Wang. "Optimal Power Control Under Interference Temperature Constraints in Cognitive Radio Network". In IEEE Wireless Communications and Networking Conference, 2007. WCNC 200\%, pages 116-120, March 2007.

[10] H.Li, Y.Gai, Z.He, K.Niu, and W.Wu. "Optimal Power Control Game Algorithm for Cognitive Radio Networks with Multiple Interference Temperature Limits". In IEEE Vehicular Technology Conference, 2008. VTC Spring 2008., pages 1554 -1558 , April 2008.

[11] L.Qian, X.Li, J. Attia, and Z. Gajic. "Power Control for Cognitive Radio Ad Hoc Networks". In 15th IEEE Workshop on Local Metropolitan Area Networks, 2007. LANMAN 200\%, pages 7 -12, June 2007.

[12] N. Shibata, K. Yamamoto, H. Murata, and S. Yoshida. "Joint Effect of Power Control, Access Control, and Multi-Hop Transmission on Area Spectral Efficiency of Cognitive Radio System". In 6th International Conference on Information, Communications Signal Processing, 2007, pages 1 -5, Dec. 2007. 
[13] N.Hao and S.J.Yoo. "ANCPC: Adaptive Neighbor Coordinated Interference Avoidance Power Control for Cognitive Radio Ad Hoc Networks". In 7th IEEE Consumer Communications and Networking Conference (CCNC), 2010, pages 1 -6, Jan. 2010.

[14] M.S. Medrano, M.B. Trindade, N.S.A. de Chaves, M.D. Fernandez, and H.J.M. Filho. "An Optimization Model for MPLS Networks". In 11th International Telecommunications Network Strategy and Planning Symposium. NETWORKS 2004, pages 285 - 290, June 2004.

[15] S. Sarkar, H.H.Yen, S. Dixit, and B. Mukherjee. "A Mixed Integer Programming Model for Optimum Placement of Base Stations and Optical Network Units in a Hybrid Wireless-Optical Broadband Access Network (WOBAN)". In IEEE Wireless Communications and Networking Conference, 2007.WCNC 2007., pages 3907 -3911, March 2007.

[16] G.Shen and R.S. Tucker. "Energy-Minimized Design for IP Over WDM Networks". IEEE/OSA Journal of Optical Communications and Networking,, 1(1):176 -186, June 2009.

[17] S. Boyd and L. Vandenberghe. "Convex Optimization". Cambridge University Press, March 2004.

[18] M. Brusco and S. Stahl. "Branch-and-Bound Applications in Combinatorial Data Analysis (Statistics and Computing)". Springer, 1 edition, July 2005.

[19] A.H. Mohsenian-Rad and V.W.S. Wong. "Joint Logical Topology Design, Interface Assignment, Channel Allocation, and Routing for Multi-Channel Wireless 
Mesh Networks". IEEE Transactions on Wireless Communications,, 6(12):4432 -4440, December 2007.

[20] F.W. Glover and G.A. Kochenberger. "Handbook of Metaheuristics (International Series in Operations Research $\&$ Management Science)". Springer, January 2003.

[21] FCC. Spectrum Policy Task Force Report, November 2002.

[22] FCC. www.wireless.fcc.gov.

[23] IEEE WRANs. www.ieee802.org/22/.

[24] C. Stevenson, G. Chouinard, Z. Lei, H.Hu, S. Shellhammer, and W. Caldwell. "IEEE 802.22: The First Cognitive Radio Wireless Regional Area Network Standard". IEEE Communications Magazine,, 47(1):130 -138, January 2009.

[25] C. Cordeiro, K. Challapali, D. Birru, and N. Sai Shankar. "IEEE 802.22: The First Worldwide Wireless Standard Based on Cognitive Radios". First IEEE International Symposium on New Frontiers in Dynamic Spectrum Access Networks, 2005. DySPAN 2005. 2005, pages 328-337, December 2005.

[26] C-H. Ko and H-Y. Wei. "Game Theoretical Resource Allocation for Inter-BS Coexistence in IEEE 802.22". IEEE Transactions on Vehicular Technology,, 59(4):1729-1744, May 2010.

[27] I.F. Akyildiz, W.Y. Lee, M.C. Vuran, and S. Mohanty. "A Survey on Spectrum Management in Cognitive Radio Networks". IEEE Communications Magazine,, 46(4):40-48, April 2008. 
[28] V. Rodriguez, K. Moessner, and R. Tafazolli. "Auction Driven Dynamic Spectrum Allocation: Optimal Bidding, Pricing and Service Priorities for Multi-Rate, Multi-Class CDMA". In IEEE 16th International Symposium on Personal, Indoor and Mobile Radio Communications, 2005. PIMRC 2005., volume 3, pages 1850 -1854 Vol. 3, September 2005.

[29] W.Ni and I.B. Collings. "Centralized Inter-Network Spectrum Sharing with Opportunistic Frequency Reuse". In IEEE Global Telecommunications Conference, 2009. GLOBECOM 2009., pages 1 -6, Nov. 2009.

[30] T. Yamada, D. Burgkhardt, I. Cosovic, and F.K. Jondral. "Resource Distribution Approaches in Spectrum Sharing Systems". EURASIP J. Wirel. Commun. Netw., 2008:1-15, 2008.

[31] T.A. Weiss and F.K. Jondral. "Spectrum Pooling: An Innovative Strategy for The Enhancement of Spectrum Efficiency". IEEE Communications Magazine,, 42(3):S8 - 14, March 2004.

[32] J. Huang, R.A. Berry, and M.L. Honig. "Auction-Based Spectrum Sharing". Mob. Netw. Appl., 11(3):405-418, 2006.

[33] C. Kloeck, H. Jaekel, and F. Jondral. "Auction Sequence as a New Resource Allocation Mechanism". In IEEE 62nd Vehicular Technology Conference, 2005. VTC-2005-Fall. 2005, volume 1, pages 240 - 244, September 2005.

[34] Y.Y. Lin and K.C. Chen. "Distributed Spectrum Sharing in Cognitive Radio Networks - Game Theoretical View". In 7th IEEE Consumer Communications and Networking Conference (CCNC), 2010, pages 1-5, January 2010. 
[35] D. Niyato and E. Hossain. "Competitive Spectrum Sharing in Cognitive Radio Networks: A Dynamic Game Approach". IEEE Transactions on Wireless Communications, , 7(7):2651 -2660, July 2008.

[36] D. Niyato and E. Hossain. "A Noncooperative Game-Theoretic Framework for Radio Resource Management in 4G Heterogeneous Wireless Access Networks". IEEE Transactions on Mobile Computing,, 7(3):332 -345, March 2008.

[37] J.E. Suris, L.A. Dasilva, Z.Han, A.B. Mackenzie, and R.S. Komali. "Asymptotic Optimality for Distributed Spectrum Sharing Using Bargaining Solutions". IEEE Transactions on Wireless Communications, 8(10):5225 -5237, October 2009.

[38] K.R. Chowdhury and I.F. Akyildiz. "Cognitive Wireless Mesh Networks with Dynamic Spectrum Access". IEEE Journal on Selected Areas in Communications, 26(1):168-181, Jan. 2008.

[39] L.Cao and H.Zheng. "Distributed Rule-Regulated Spectrum Sharing". IEEE Journal on Selected Areas in Communications,, 26(1):130 -145, Jan. 2008.

[40] L. Toka, A. Korosi, and A. Vidacs. "On Distributed Dynamic Spectrum Allocation for Sequential Arrivals". In IEEE Symposium on New Frontiers in Dynamic Spectrum, 2010, pages 1 -12, April 2010.

[41] F. Bernardo, R. Agusti, J. Perez-Romero, and O. Sallent. "Distributed Spectrum Management Based on Reinforcement Learning". In 4th International Conference on Cognitive Radio Oriented Wireless Networks and Communications, 2009. CROWNCOM '09., pages 1 -6, June 2009. 
[42] J.E. Suris, L.A. DaSilva, Zhu Han, and A.B. MacKenzie. "Cooperative Game Theory for Distributed Spectrum Sharing". IEEE International Conference on Communications, 2007. ICC '07., pages 5282-5287, June 2007.

[43] J.Case. "A Class of Games Having Pareto Optimal Nash Equilibria". IEEE Journal on Selected Areas in Communications,, 13(3), 1974.

[44] A. Attar, M.R. Nakhai, and A.H. Aghvami. "Cognitive Radio Game for Secondary Spectrum Access Problem". IEEE Transactions on Wireless Communications, 8(4):2121 -2131, April 2009.

[45] E. Kalai. "Game theory: Analysis of conflict : By Roger B. Myerson, Harvard Univ. Press, Cambridge, MA, 1991. 568 pp.,". Games and Economic Behavior, 3(3):387-391, August 1991.

[46] M. Adibi and V.T. Vakili. "Comparison of Cooperative and Non-Cooperative Game Schemes for SINR-Constrained Power Allocation in Multiple Antenna CDMA Communication Systems". In IEEE International Conference on Signal Processing and Communications, 2007. ICSPC 2007, pages $1151-1154$, Nov. 2007.

[47] Y.Yi, J.Zhang, Q.Zhang, T.Jiang, and J.Zhang. "Cooperative CommunicationAware Spectrum Leasing in Cognitive Radio Networks". In IEEE Symposium on New Frontiers in Dynamic Spectrum, 2010, pages 1 -11, April 2010.

[48] C.G Yang, J.D.Li, and Z.Tian. "Optimal Power Control for Cognitive Radio Networks Under Coupled Interference Constraints: A Cooperative Game-Theoretic 
Perspective". IEEE Transactions on Vehicular Technology,, 59(4):1696 -1706, May 2010.

[49] O. Yu and E. Saric. "Non-Cooperative Optimal Game-Theoretic Opportunistic Dynamic Spectrum Access". In Sixth International Conference on Broadband Communications, Networks, and Systems, 2009. BROADNETS 2009., pages 1 -8, September 2009.

[50] G. Alyfantis, G. Marias, S. Hadjiefthymiades, and L. Merakos. "Non-Cooperative Dynamic Spectrum Access for CDMA Networks". In IEEE Global Telecommunications Conference, 200\%. GLOBECOM '07., pages 3574-3578, November 2007.

[51] D. Niyato and E. Hossain. "Competitive Pricing for Spectrum Sharing in Cognitive Radio Networks: Dynamic Game, Inefficiency of Nash Equilibrium, and Collusion". IEEE Journal on Selected Areas in Communications,, 26(1):192 202, January 2008.

[52] F. Capar and F. Jondrai. "Spectrum Pricing for Excess Bandwidth in Radio Networks". In 15th IEEE International Symposium on Personal, Indoor and Mobile Radio Communications, 2004. PIMRC 2004., volume 4, pages 2458 2462 Vol.4, September 2004.

[53] N.Li, L.Gong, and S.Li. "Price-Based Spectrum-Allocation Relay Routing in Cognitive Radio Networks". In 7th International Conference on Information, Communications and Signal Processing, 2009. ICICS 2009., pages 1-5, December 2009 .

[54] "www.SpecEx.com". 
[55] D. Niyato, E. Hossain, and Long Le. "Competitive Spectrum Sharing and Pricing in Cognitive Wireless Mesh Networks". IEEE Wireless Communications and Networking Conference, 2008. WCNC 2008., pages 1431-1435, April 2008.

[56] A. Capone, J. Elias, and F. Martignon. "Optimal Design of Service Overlay Networks". 4th International Telecommunication Networking Workshop on QoS in Multiservice IP Networks, 2008. IT-NEWS 2008., pages 46-52, Feb. 2008.

[57] R.C. Pereira, R.D. Souza, and M.E. Pellenz. "Using Cognitive Radio for Improving the Capacity of Wireless Mesh Networks". IEEE 68th Vehicular Technology Conference, 2008. VTC 2008-Fall., pages 1-5, Sept. 2008.

[58] C.Han J.Chen, C. Liao and S.Li. "Extending Available Spectrum in Cognitive Radio: Hierarchical Spectrum Sharing Network". Computer Networks, 52(4):850-863, March. 2008.

[59] A. Rad and V. Wong. "Joint Logical Topology Design, Interface Assignment, Channel Allocation, and Routing for Multi-channel Wireless Mesh Networks". IEEE Transactions on Wireless Communications, 6(12):4432-4440, 2007.

[60] V. Rajakumar, M. N. Smadi, S. C. Ghosh, T. D. Todd, and S. Hranilovic. "Interference Management in WLAN Mesh Networks Using Free-Space Optical Links". Journal of Lightwave Technology,, 26(13):1735-1743, July1, 2008.

[61] M.Ma and D.H.K. Tsang. "Joint Spectrum Sharing and Fair Routing in Cognitive Radio Networks". In 5th IEEE Consumer Communications and Networking Conference, 2008. CCNC 2008., pages 978-982, Jan. 2008. 
[62] Y.Shi, Y.T. Hou, and S. Kompella. "A Cross-Layer Approach to Multi-Hop Networking with Cognitive Radios". In IEEE Military Communications Conference, 2008. MILCOM 2008., pages 1-7, Nov. 2008.

[63] A.H.Mohsenian-Rad. "Optimal Resource Management in Wireless Access Networks ". PHD Thesis, University of British Colombia, April. 2008.

[64] Y.Lebbah, C.Michel, and M.Rueher. "An Efficient and Safe Framework for Solving Optimization Problems". Journal of Computational and Applied Mathematics, 199(2):372 - 377, 2007. Special Issue on Scientific Computing, Computer Arithmetic, and Validated Numerics (SCAN 2004).

[65] Y.Huang, G.Zhang, and J.Wang. "An Optimization Dijkstra Algorithm Based on Two-Function Limitation Strategy". In 2nd International Congress on Image and Signal Processing, 2009. CISP '09., pages 1 -4, Oct. 2009. 